\title{
Theoretical Analysis of the Micro-optic Bottle Resonator and its Applications
}

by

Yusra Jat

A thesis submitted to the Faculty of Graduate and Postdoctoral Affairs in partial fulfillment of the requirements for the degree of

Master of Applied Science

in

Electrical and Computer Engineering

Carleton University

Ottawa, Ontario

(C) 2021, Yusra Jat 


\begin{abstract}
The micro-optic resonators confines light in a small volume resonating cavity and sustains a high-quality factor. Among all micro resonator morphologies, the micro-optic bottle resonator has a 3-D mode confinement geometry. In this thesis, MBR is profiled by azimuthal sculpting of a pair of rings along the glass whisker's perimeter. The structure is examined using a numerical solver optimized for the cylindrical symmetry of such resonators. The modal space and field profiles are computed as a function of ring spacing and demonstrate that multiple glass region confined states are available and can generally be thought of as Whispering Gallery Modes. Additional computational results are presented when the structure is configured as a sensor, suitable for measuring a specific constituent such as index of refraction, temperatures, gases, and chemicals. Attempts were underway to fabricate the MBR's geometry, but the work has remained incomplete due to COVID-19.

In this thesis from designing the MBR (reduced-size) to implementing its fundamental mode WGM resonance wavelength into various sensor-related applications (mentioned above) are the work of the author. Whereas for the computational needs FFB mode solver was built by the supervisor (Dr. Gauthier).
\end{abstract}




\section{Acknowledgements}

I want to thank my thesis supervisor, Dr. Robert Gauthier, for providing me with an opportunity to work in this area of photonics. It was a great pleasure to work under his supervision and guidance.

I would also like to thank the microfabrication lab staff, Rob Vandusen, Angela Williams, and Rodney Aiton, for training, consultation, and fabrication and equipment operation assistance. I want to thank Professor Luc Levesque from the Royal Military College. I would also like to thank undergrad student Tauheed Elahi who worked for me, and helped me during the fabrication process.

Finally, I express deep gratitude to my parents, my siblings, my husband Uzair Rehman, and my son Ariz Rehman for being my continuous support in this journey. Without their prayers and well wishes, this wouldn't have been possible. 


\section{Table of Contents}

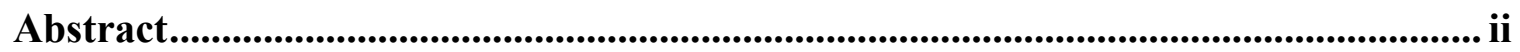

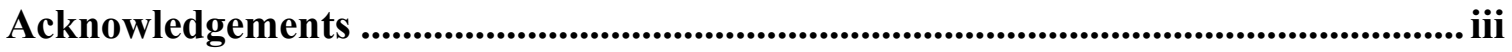

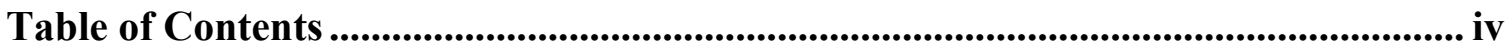

List of Tables ...................................................................................................................... ix

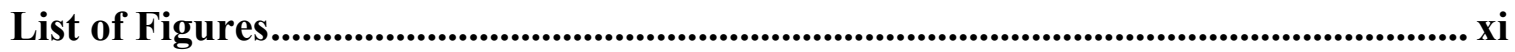

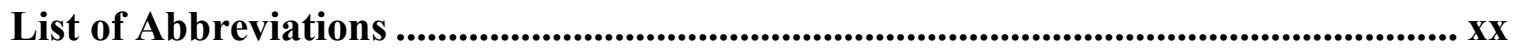

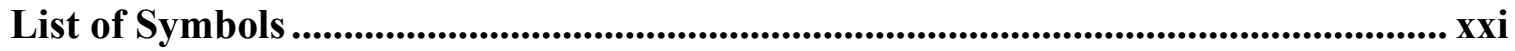

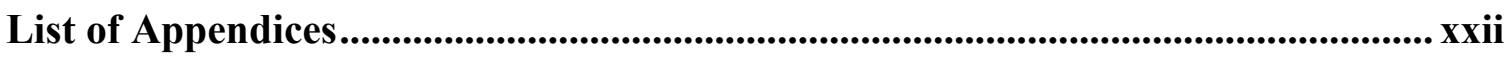

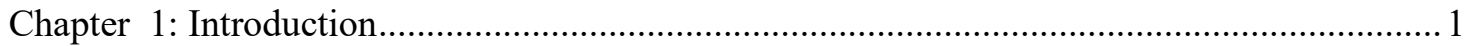

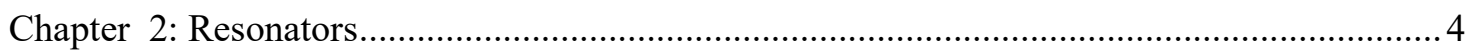

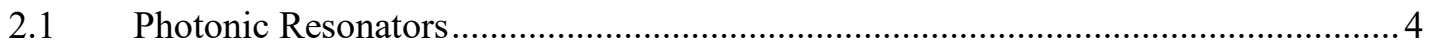

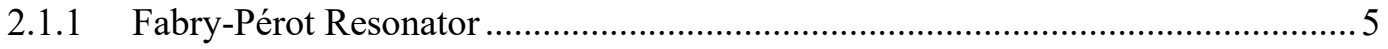

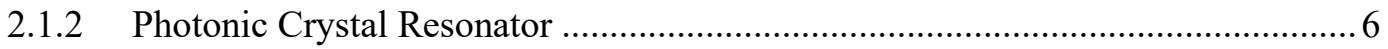

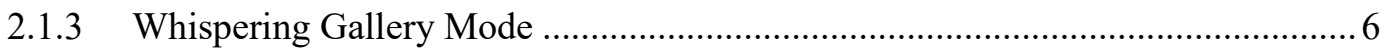

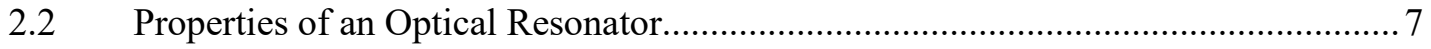

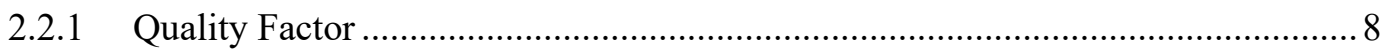

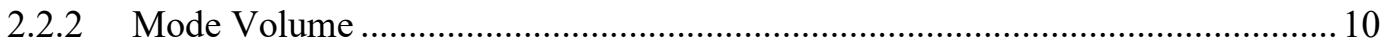

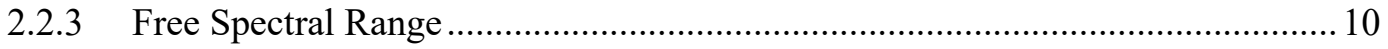




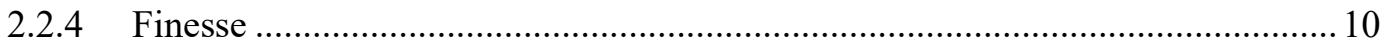

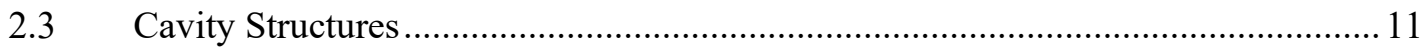

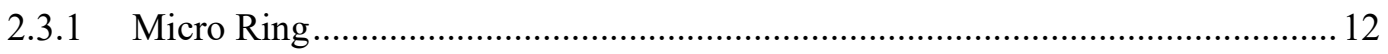

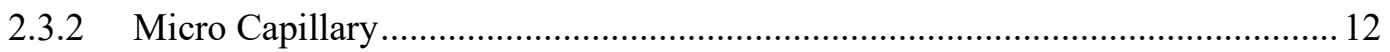

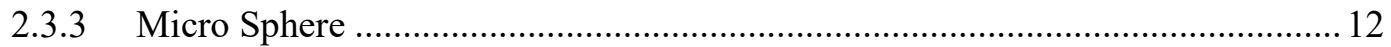

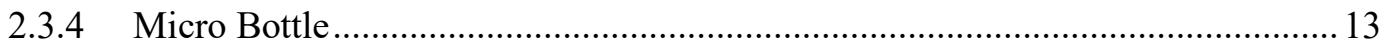

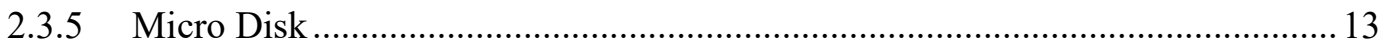

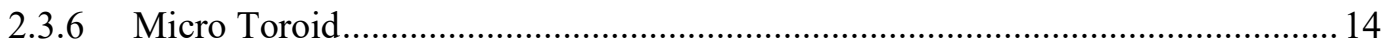

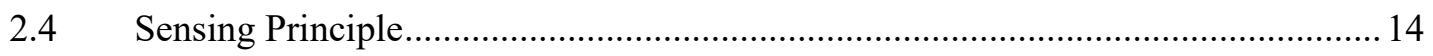

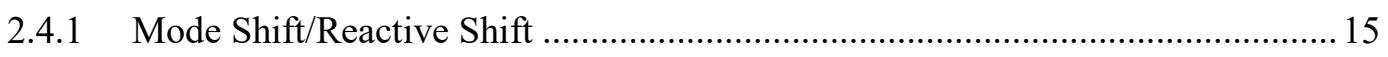

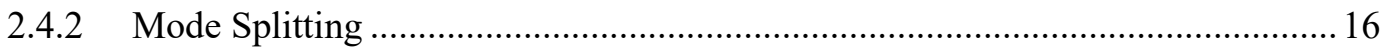

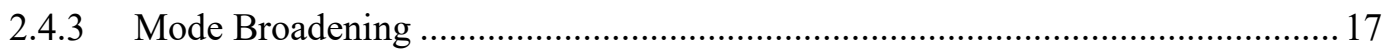

Chapter 3: Literature Review on the Micro-optic Bottle Resonator.......................................21

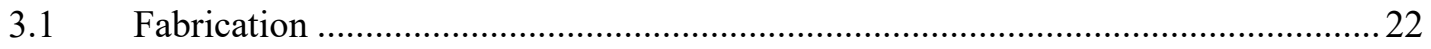

3.1.1 Melting Optical Glass Fiber by a $\mathrm{CO}_{2}$ Laser ...............................................22

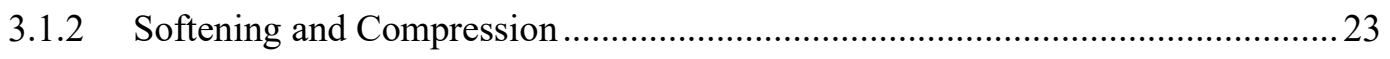

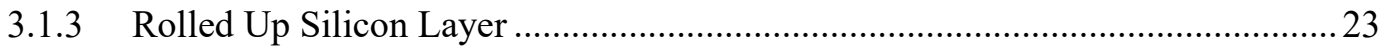

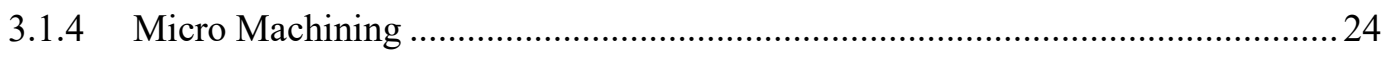

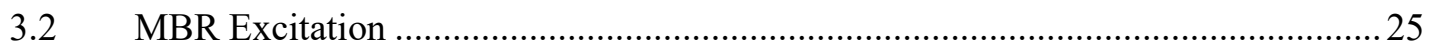




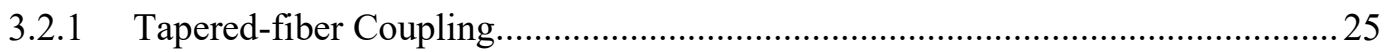

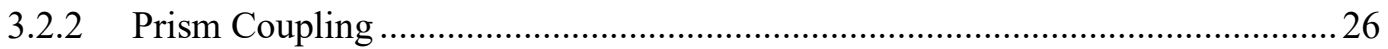

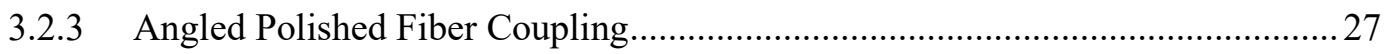

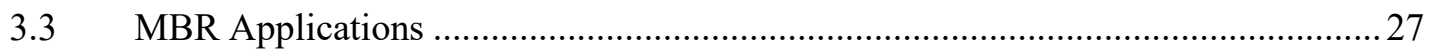

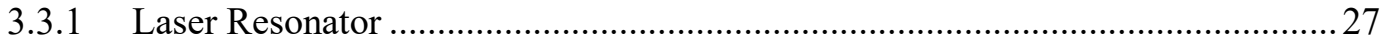

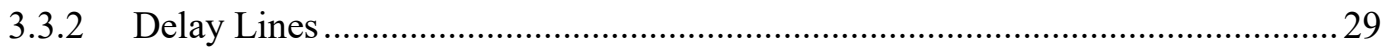

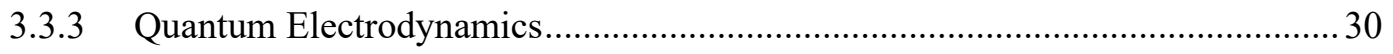

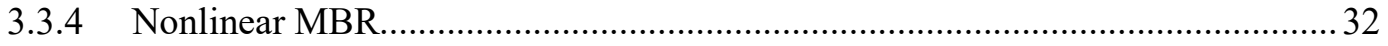

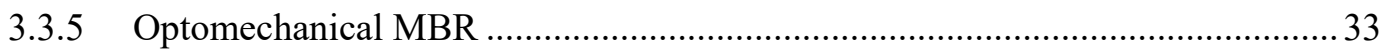

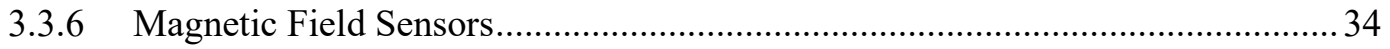

3.4 Hollow Micro-optic Bottle Resonator (HMBR) ................................................... 36

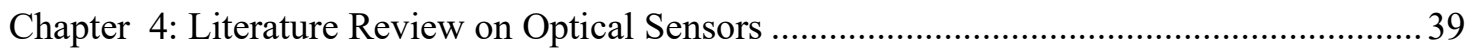

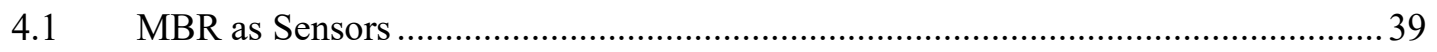

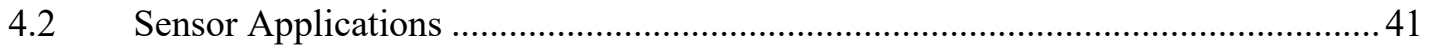

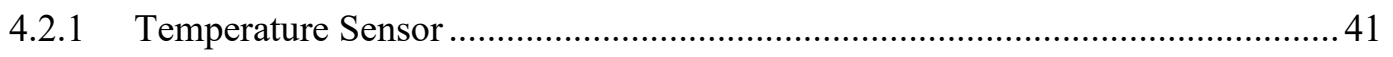

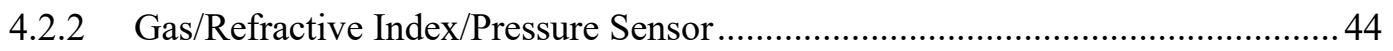

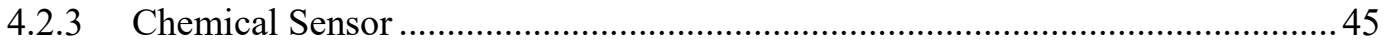

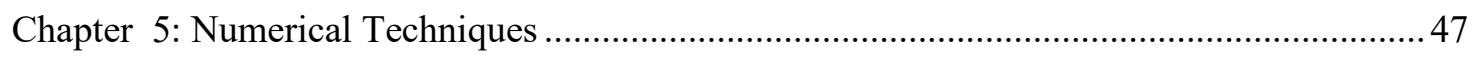

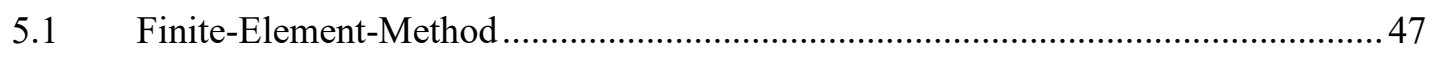


5.2 Finite-Difference Time-Domain

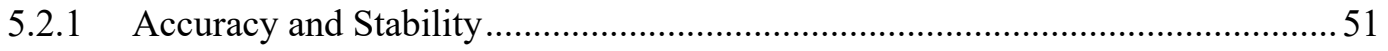

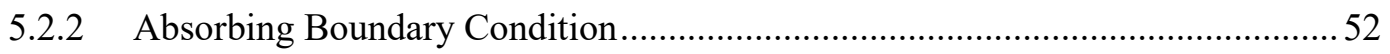

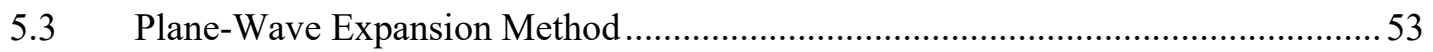

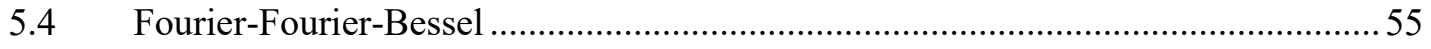

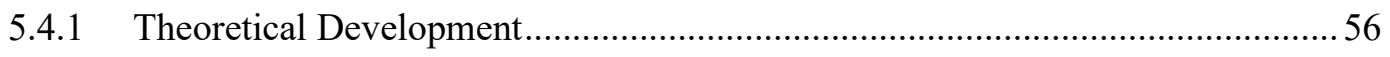

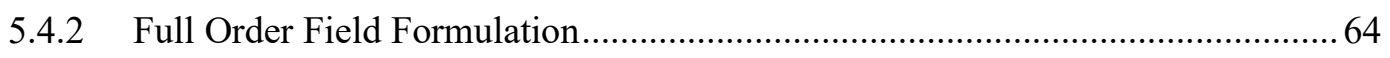

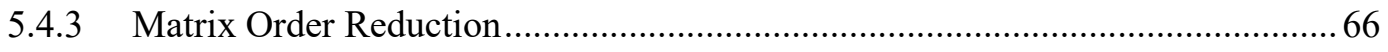

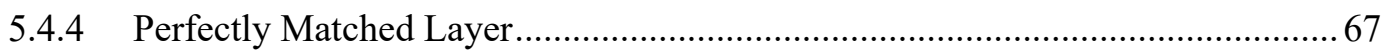

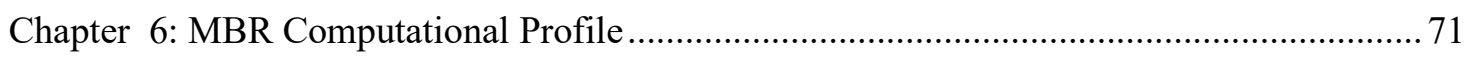

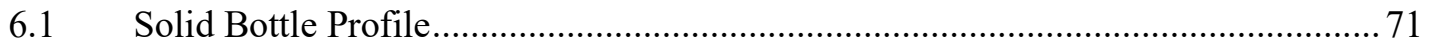

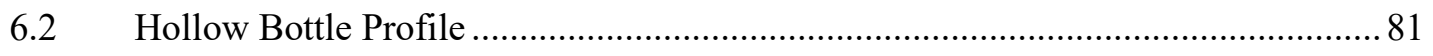

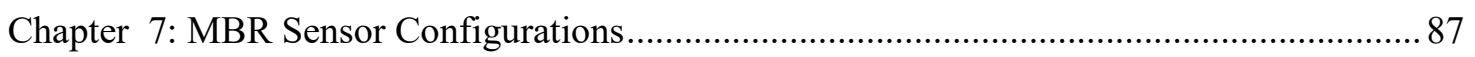

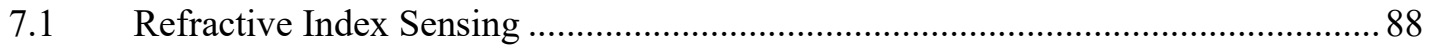

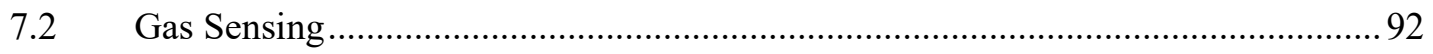

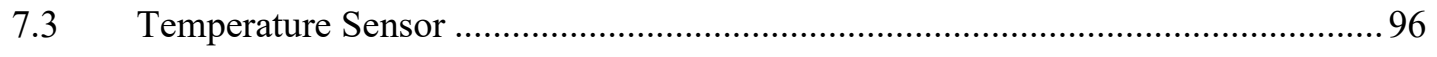

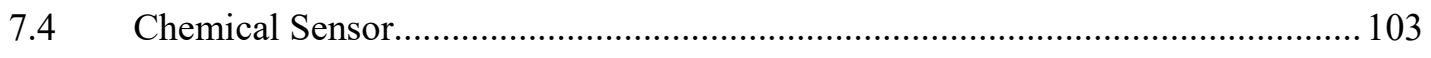

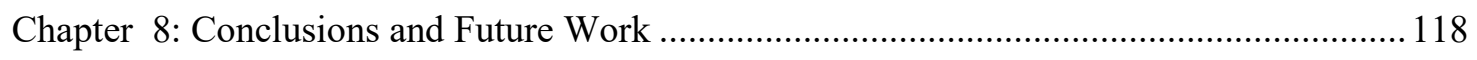

Appendices.......................................................................................................................... 120 


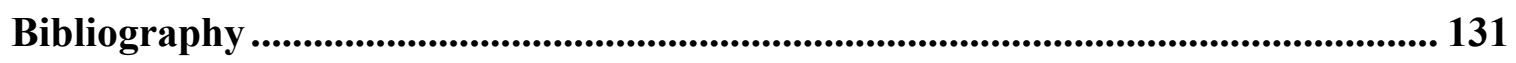




\section{List of Tables}

Table 7.1 The refractive index and its corresponding mode zero wavelengths for the central and upper bottle regions.

Table 7.2 The sensitivities calculated over each zone for the central and upper bottle regions.

Table 7.3 Wavelength and refractive index as a function of low pressure. 93

Table 7.4 Wavelength and refractive index as a function of pressure in the high pressure domain

Table 7.5 Refractive index as a function of temperature, which highlights the thermooptic effect as a change in wavelength.

Table 7.6 Refractive index as a function of temperature change for $\mathrm{MBR}$ and $\mathrm{CO}_{2}$ gas (at $600 \mathrm{psi}$ ) causes the wavelength to shift 101

Table 7.7 Refractive index as a function of temperature change for the $\mathrm{MBR}$ and $\mathrm{CO}_{2}$ gas at 1500 psi causes the wavelength to shift. 102

Table 7.8 Wavelength shift as a function of refractive index. 106

Table 7.9 Refractive index as a function of the concentration of liquid benzene 108

Table 7.10 Refractive index and wavelength as a function of chlorobenzene concentration 110 
Table 7.11 Wavelength change as a function of refractive index in the inner-outer regions of HMBR.

Table 7.12 For comparison, the refractive index change and wavelength variations related to the inner-outer region and inner region. 115

Table A.3.1 Summary of the main steps followed during the photolithography process. 


\section{List of Figures}

Figure 2.1 Three photonic resonator structures are displayed to compare how photons are confined inside. On the left is a FP resonator, the center one is PCs, and the right one is

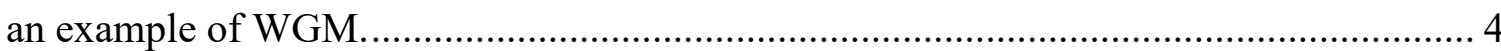

Figure 2.2 The resonance spectrum of a Fabry-Pérot Resonator................................. 6

Figure 2.3 The resonator's Quality factor in the frequency domain.............................. 9

Figure 2.4 Geometrical representation of commonly used micro-cavity/resonator structures based on the WGM principle (a) micro-ring, (b) micro-capillary, (c) microsphere, (d) micro-bottle, (e) micro-disk, and (f) micro-toroid. 11

Figure 2.5 The dotted line is the original spectrum. The solid curve is the mode shift after the analyte is introduced in the resonator surrounding. 16

Figure 2.6 Mode splitting sensing modality: the dotted line is the original spectrum, and the solid curve represents the mode splitting after the analyte is introduced onto the resonator surface.

Figure 2.7 In the mode broadening sensing modality, the dotted line is the original spectrum. The solid curve shows the broadened mode when the analyte is placed on to

the resonator's surface 18 
Figure 2.8 Transmission spectrum of 0 to 4 nanoparticles attached to the surface excited by a visible light laser. The figure is reproduced with permission from [38]..... 19

Figure 3.1 Schematic profile of the Micro-optic Bottle Resonator. 22

Figure 3.2 MBR fabrication either by $\mathrm{CO}_{2}$ laser or by a softening-compression method. Here, $L_{b}$ is the axial width, $D_{b}$ is the bottle diameter, and $D_{s}$ is the tapered end's diameter.

Figure 3.3 The bottle resonator's schematic exhibiting a parabolic lobe on the outer rolling edge is on the left. A schematic of the sample fabrication is on the right.

Figure 3.4 The MBR is micromachined by a mask-less laser writing machine on the fiber using a photolithographic process. 25

Figure 3.5 The MBR is coupled to tapered-fiber. Reproduced with permission from [45]

Figure 3.6 MBR coupled to the prism. 26

Figure 3.7 MBR coupled to an angled-end (polished) fiber. 27

Figure 3.8 The principle of single-mode WGM lasing in a polymer micro-resonator. (a) shows the multimode lasing behaviour and (b) shows the engineered pump intensity can modify the spatial gain profile of bottle WGM, enabling single-mode lasing. This figure has been reproduced with permission [48]. 28

Figure 3.9 (a) Illustration of an optical bottle resonator delay line. Light is coupled into the resonator from a transverse waveguide (microfiber) and experiences WGM 
propagation along the resonator surface. The inset shows the magnified profile of the fiber radius variation. (b) Semi parabolic variation of a bottle resonator radius used in the numerical simulations. The figure has been reproduced with permission from [50] ...... 30

Figure 3.10(A) Schematic of MBR and single Rb atom coupled with two input-output microfibers. (B \& C) Port to port transmission vs normalized field decay of fiber coupled resonator. The solid circles indicate transmission in the forward direction and open circles indicate transmission in the backward direction. The figure is reproduced with permission from [51]

Figure 3.11 Schematic of an add-drop coupling configuration using two ultra-thin coupling fibers. The spiraling red line in the resonator traces the ray path. This figure has been reproduced with permission from [52] ............................................................ 33

Figure 3.12 An interaction of optical and mechanical modes via radiative pressure of photons. The coupling between the optical pump mode and mechanical mode creates the Stokes and Anti-Stokes fields. The figure has been reproduced with permission of [10].34

Figure 3.13 (a) Opto-fluidic MBR filled with magnetic fluid coupled to a tapered fiber. COMSOL simulations of field distribution of modes in the xy (b) and yz (c) planes. Schematic diagram of magnetic fluid particles without (d) and with applied magnetic field (e). The figure has been reproduced with permission of [56]. 35

Figure 3.14 A general schematic of a hollow micro-optic bottle resonator. The dotted line represents the inner radius, creating a boundary between the thin walls and hollowed

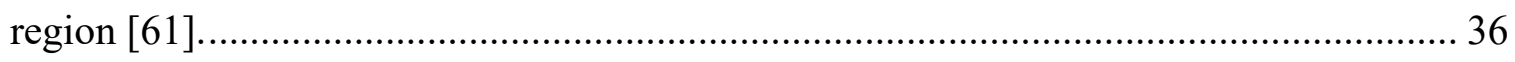


Figure 3.15 The principle of optomechanical fluid detection of particle flowing through hollow micro capillary resonator. This image has been reproduced with permission from

Figure 3.16 The mode is confined in the thin-walls, its evanescent fields interact with the external analyte (A). The HMBR evanescent fields also leak into the inner-core where it interacts with the analyte (B). 38

Figure 4.1 The coverage factor, $\mathrm{k}=3$, gives $99.7 \%$ certainty that the minimum analyte is present. . 40

Figure 5.1 Basic finite elements are shown in (a) one-dimension, (b) two-dimensions, and (c) three-dimensions 48

Figure 5.2 The Yee cell for TM polarization. The $E$ and $H$ fields are discretized on overlapping grids and used in finite difference expressions of Maxwell's curl equations [78]. 51

Figure 5.3 Cylindrical computational domain. 57

Figure 6.1 Left is the geometry and parameter identification of the MBR used in the numerical computations and sensor configurations. The structure is periodic in the axial direction with three periods shown (center), and uniform under azimuthal rotation. The right figure shows the reconstruction of the $(1,1)$ relative permittivity tensor element showing that the original bottle resonator is obtained and ensures convergence in the spectral decomposition. 
Figure 6.2 The wavelength's real part of computed states versus matrix solution indexing is indicated here. States identified with a ( $\bullet$ ) correspond to modes highly localized to the high permittivity glass bottle region. 75

Figure 6.3 The wavelength's imaginary part of the computed states versus matrix solution indexing is indicated here. Bottle confined states have negligible field components in the PML region, making the imaginary part very close to zero. 77

Figure 6.4 The resonator states obtained for the structure in Figure 6.1 at the wavelengths identified in Figure 6.2 when the bottle parameter, $\mathrm{W}$, was $1.5 \mu \mathrm{m}$. The top set shows the intensity profile in the $(\mathrm{r}, \mathrm{z})$ plane for states localized in the glass cylinder's central region. The bottom set shows the intensity profile for states localized in the upper and lower regions resulting from the additional bottle produced through axial periodicity... 78

Figure 6.5 The lowest order mode in 3-D representation demonstrating the azimuthal mode order of 20 (40 intensity maximum over $2 \pi$ rotation). 79

Figure 6.6 Plot of the bottle confined resonator states as a function of the resonator width, $\mathrm{W}$ is displayed. At $\mathrm{W}=2.5 \mu \mathrm{m}$, the central and upper bottles are identical. For $\mathrm{W}<2.5$ $\mu \mathrm{m}$, the central bottle is narrower than the upper bottle making the lower confined states exhibit a smaller wavelength. The structure with $\mathrm{W}=1.5 \mu \mathrm{m}$ was selected for sensor consideration. 80

Figure 6.7 The basic geometry of the hollow micro-optic bottle resonator where an additional notch was created inside the central bottle region. The distance of rings from

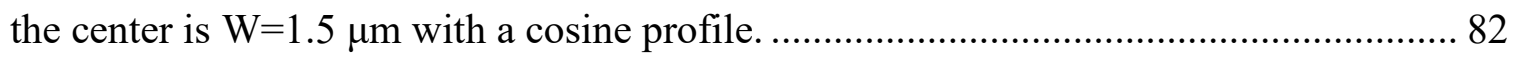


Figure 6.8 The resonator states obtained for the structure of Figure 6.7 at the wavelengths identified in Figure 6.9 when the bottle parameter, W, is $1.5 \mu \mathrm{m}$. The top set shows the intensity profile in the $(r, z)$ plane for states localized in the central bottle region's thinwalls. The bottom set shows the intensity profile for states localized in the upper and lower regions resulting from the additional bottle produced through axial periodicity. .. 83

Figure 6.9 The real part of the wavelength for the HMBR's computed states versus matrix solution indexing. States identified with a $(\bullet \mathbf{m})$ correspond to modes highly localized to the bottle region with a high permittivity glass cylinder. 85

Figure 6.10 The imaginary part of the wavelength for the HMBR's confined states has negligible field components in the PML region making the imaginary part very close to

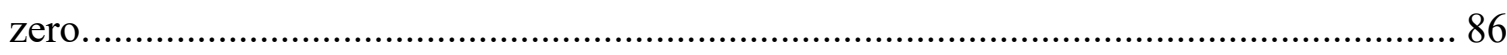

Figure 7.1 The bottle confined mode wavelength response as a function of the ambient medium's index of refraction / relative permittivity for the lowest-order mode axially directed in the upper bottle. It is denoted by $(\checkmark)$ in the central bottle region and ( $\mathbf{\square})$ for azimuthal order 20 .

Figure 7.2 Changes in $\mathrm{CO}_{2}$ pressure (low range) versus wavelength shift. From the graph, the sensitivity of $79.6 \mathrm{~nm} / \mathrm{RIU}$ was calculated. 93

Figure 7.3 Changes in $\mathrm{CO}_{2}$ pressure (high range) versus the shift in wavelength.

Sensitivity was found to be $118.2 \mathrm{~nm} / \mathrm{RIU}$. 95 
Figure 7.4 The solid line represents the thermo-optic effect, where the wavelength varies as a function of $10^{\circ} \mathrm{C}$ temperature changes at each point. The overall change in temperature will become $140{ }^{\circ} \mathrm{C}$. 98

Figure 7.5 Changes in wavelength of $\mathrm{CO}_{2}$ gas at 600 psi with varying temperature. The sensitivity was determined to be $4.9 \mathrm{~nm} /{ }^{\circ} \mathrm{C}$ 101

Figure $7.6 \mathrm{CO}_{2}$ gas at 1500 psi with varying temperature versus wavelength gives $4.8 \mathrm{~nm} /{ }^{\circ} \mathrm{C}$ sensitivity 102

Figure 7.7 The refractive index (n) variation is visualized in the central bottle. When the $n$ value of the hollow notch gets close enough to that of the optical glass at $n=1.5$ and $n$ $=1.55$, an increase in the intensity of the confinement optical mode is observed 105

Figure 7.8 Bottle confined resonator state wavelength response as a function of the internal hollow region's refractive index change (lowest order mode axially directed). 106 Figure 7.9 The sensitivity of a chemical sensor for a generalized range concerning the next point is identified. 107

Figure 7.10 As the concentration of benzene changes, the refractive index changes causing a shift in the wavelength, and a sensitivity of $123.8 \mathrm{~nm} / \mathrm{RIU}$ was achieved.... 109

Figure 7.11 The concentration of liquid chlorobenzene with the first-order mode wavelength change gives a $185.9 \mathrm{~nm} / \mathrm{RIU}$ sensitivity. 111 
Figure 7.12 The lowest-order mode (axially directed) confined in the thin wall of the HMBR. Its evanescent fields interact with the inner and outer medium's refractive index.

Figure 7.13 Bottle confined resonator state wavelength response as a function of the internal and external region's refractive index change for the lowest order mode in the axial direction. A sensitivity of $176.5 \mathrm{~nm} / \mathrm{RIU}$ was detected.

Figure 7.14 The refractive index changes from $n=1$ to 1.2 , changes the wavelengths (inner and inner-outer regions) for the lowest-order mode axially directed.

Figure A.2.1 At an exposure time of 6.5 seconds with a stage level set to 575, around 6micron ring indentations were achieved with a bottle region of 7 microns.

Figure A.3.1 The left is the front view where ring indentations (minimum $4 \mu \mathrm{m}$ ) are created and the right is the back view of the fiber.

Figure A.3.2 Lab fabricated solid micro-optic bottle resonator using SU-8 (negative resist) dip-coated twice.

Figure A.3.3 The dimension achieved on a dipped coated fiber after going through the photolithographic process was $\sim 30 \mu \mathrm{m}$ wide.

Figure A.3.4 Another attempt was made to create different size ring indentations with a varying width of the bottle resonator. Sprayed SU-8 while rotating the fiber 17 seconds exposed by laser writing machine, SF-100. 125 
Figure A.3.5 Heated and Spray coated SU-8 was deposited and exposed through the Aligner. 126

Figure A.3.6 Left side is the front view of the fiber just as it was placed in the aligner and the right side is the edge view, hard baked to finish the photolithographic process....... 127

Figure A.3.7 $\mathrm{O}_{2}$ Plasma preen heated SU-8-2 is sprayed using SF-100 for exposure.... 128 


\section{List of Abbreviations}

\begin{tabular}{|c|c|}
\hline MBR & Micro Bottle Resonator \\
\hline WGM & Whispering Gallery Mode \\
\hline TIR & Total Internal Reflection \\
\hline$Q$ & Quality factor \\
\hline$V$ & Volume \\
\hline $\mathrm{CW}$ & Clockwise \\
\hline $\mathrm{CCW}$ & Counter Clockwise \\
\hline HMBR & Hollow Micro Bottle Resonator \\
\hline FEM & Finite-Element-Method \\
\hline FDTD & Finite-Difference-Time-Domain \\
\hline PWEM & Plane Wave Expansion Method \\
\hline FFB & Fourier-Fourier-Bessel \\
\hline PML & Perfectly Matched Layers \\
\hline RIU & Refractive Index Unit \\
\hline $\mathrm{ABC}$ & Absorbing Boundary Condition \\
\hline PEB & Post Exposure Bake \\
\hline
\end{tabular}




\section{List of Symbols}

\begin{tabular}{ll}
$\Delta \lambda$ & Spectral Range \\
$\lambda$ & Wavelength \\
$\mu$ & Micron \\
$\alpha$ & Thermal Expansion Coefficient \\
$\mu_{0}$ & Permeability \\
$\varepsilon_{\mathrm{o}}$ & Permittivity \\
$\varepsilon_{r}$ & Relative Permittivity \\
$G_{n}$ & \\
\hline & Reciprocal Lattice Vector
\end{tabular}




\section{List of Appendices}

Appendix A................................................................... 120

Appendix B........................................................... 130 


\section{Chapter 1: Introduction}

The optical resonators can be configured in several basic geometries, for which the bottle resonator is a useful micro-optic variation. In its most general form, the Micro-optic Bottle Resonator (MBR) consists of an axially extended and azimuthally symmetric high relative permittivity region surrounded by a lower relative permittivity medium. The most common geometries are optical fibers, rings, and spheres [1-4]. The physical principle that confines light about the structure is generally referred to using the ray optics analogy of Whispering Gallery Modes (WGM), even when the optical wavelength is comparable to the structural dimensions. The MBR finds itself a candidate as the base structure of numerous real-world applications such as the laser resonator [5], delay line [6], narrow linewidth filter [7], quantum electrodynamics [8], nonlinear MBR [9], and optomechanical MBR [10]. In this thesis, the MBR is numerically examined using spectral analysis exploiting the structure's cylindrical symmetry to reduce the computation of a 3-D system requiring only a 2-D (radial, axial) analysis. Numerical calculations were performed using the Fourier-Fourier-Bessel (FFB) mode solver, whose matrix reduction techniques results in a faster computational process compared to Finite-Difference Time-Domain (FDTD) and Plane-Wave Expansion Method (PWEM).

The MBR of this thesis was sculpted from two azimuthally directed ring indentations on a glass fiber. The optical mode space for the MBR as a function of axial indentation ring spacing is provided, and samples of the available modes are plotted. The 
modal properties for the MBR structures acting as an optical sensor are examined when the ambient medium's refractive index, temperature, and chemical composition change.

Chapter 2 is devoted to literature review of optical resonators, in general, and review WGM resonance condition, sensing modalities, characteristics, and various cavity structures.

Chapter 3 focuses on literature review of the micro-optic bottle resonator (solid and hollow) fabrication methods, optical coupling techniques, and potential applications. A fabrication process explored for MBR fabrication was cut short due to lab access restrictions from COVID-19.

Chapter 4 gives a literature review on the micro-optic bottle resonator's sensing capabilities and detection limits when utilized to measure temperature, gas, and chemical constituents.

Chapter 5 gives an overview of numerical techniques such as FEM, FDTD, PWEM, and FFB. Each of these has its advantages and disadvantages depending on the structure, material, and properties to be determined. The suitability of the FFB mode solver for numerical computations used in this thesis is presented. The theoretical development is provided, leading to an eigenvalue equation where the optical mode complex frequencies and field profiles are the direct solution information.

Chapter 6 presents author's work on the geometrical and material properties of the micro-optic bottle resonator and hollow micro-optic bottle resonator designing. The mode 
space is computed using FFB for the designed resonators as a function of the bottle's axial width mode confinement.

Chapter 7 presents the author's theoretical results obtained from the designed solid and hollow-core micro-optic bottle resonator structure configured as optical sensors. The modal properties of the MBR structures were examined as a function of the ambient medium's refractive index and change in temperature. This chapter also presents the hollow-core bottle resonator configuration used to detect chemical variation in the internal (hollow) and external ambient regions.

Chapter 8 summarizes all findings. The results have led to one conference proceeding and an international conference poster presentation. The thesis supervisor developed the FFB mode solver. All numerical results are the work of the author.

Appendix A includes details on the MBR micromachining's fabrication process using a direct-writing machine, SF-100, and a UV-Aligner at Carleton University's micro fabrication lab facility. Due to COVID-19, the work was halted. 


\section{Chapter 2: Resonators}

\subsection{Photonic Resonators}

Photonic resonant structures are formed in device configurations where light harnessing is induced by confining photons. In general, resonant structures can be sorted into two categories: refractive and diffractive resonators [11]. Refractive resonators rely on Total Internal Reflection (TIR) of light, such as the Fabry-Pérot resonator (FP) and Whispering Gallery Mode (WGM) resonator. Diffractive resonators depend on light diffraction, such as the Photonic Crystals (PCs) resonator and Laser resonator. See Figure 2.1 for a few resonating structure examples. The principles of each category are examined below.

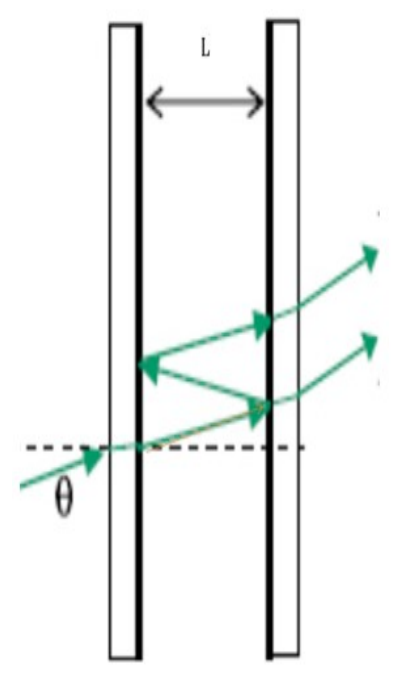

$Q \sim 10^{3}$

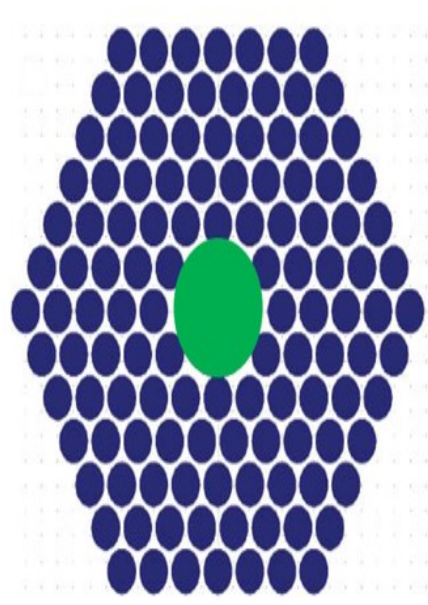

Q $10^{6}$

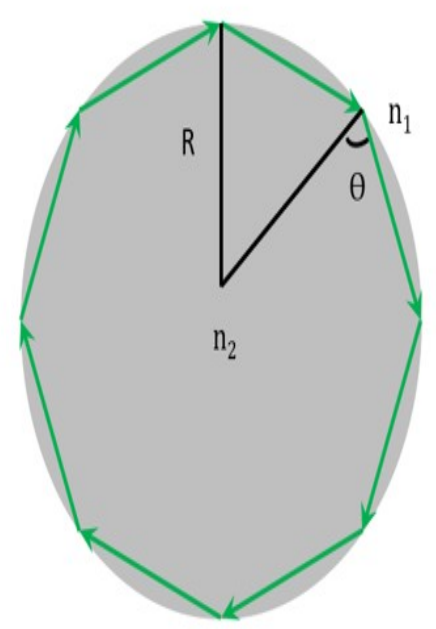

$Q^{\sim 10^{11}}$

Figure 2.1 Three photonic resonator structures are displayed to compare how photons are confined inside. On the left is a FP resonator, the center one is PCs, and the right one is an example of $W G M$. 


\subsubsection{Fabry-Pérot Resonator}

FP resonator can consist of a transparent medium bound by two parallel reflecting surfaces facing each other, as shown in Figure 2.1-left. FP resonator type can also be configured from curved mirrors. Each time light encounters one of the surfaces, a portion of it is transmitted, and the remaining is reflected. The net effect is to divide the beam into multiple beams, which then interfere together.

An incident beam travelling along the axis inside the resonator should be halfwavelength (or an integer multiple) of the resonator's length. When the optical field is trapped inside the resonator it can lead to a strong field build-up, and this phenomenon is often called resonance (Figure 2.2). The resonance is expressed as:

$$
\lambda_{r}=\frac{2 n L}{m}
$$

where $n$ is the index of refraction, $L$ is the spacing between the reflecting mirrors, and $m$ is an integer $(m=1,2,3 \ldots)$. Typical resonator parameters of interest, the Quality factor $(Q)$ and Free Spectral Range $(F S R)$, are defined in Sections 2.2.1 and 2.2.3, respectively. In general, the FP resonator displays a small $Q$ factor [1]. 


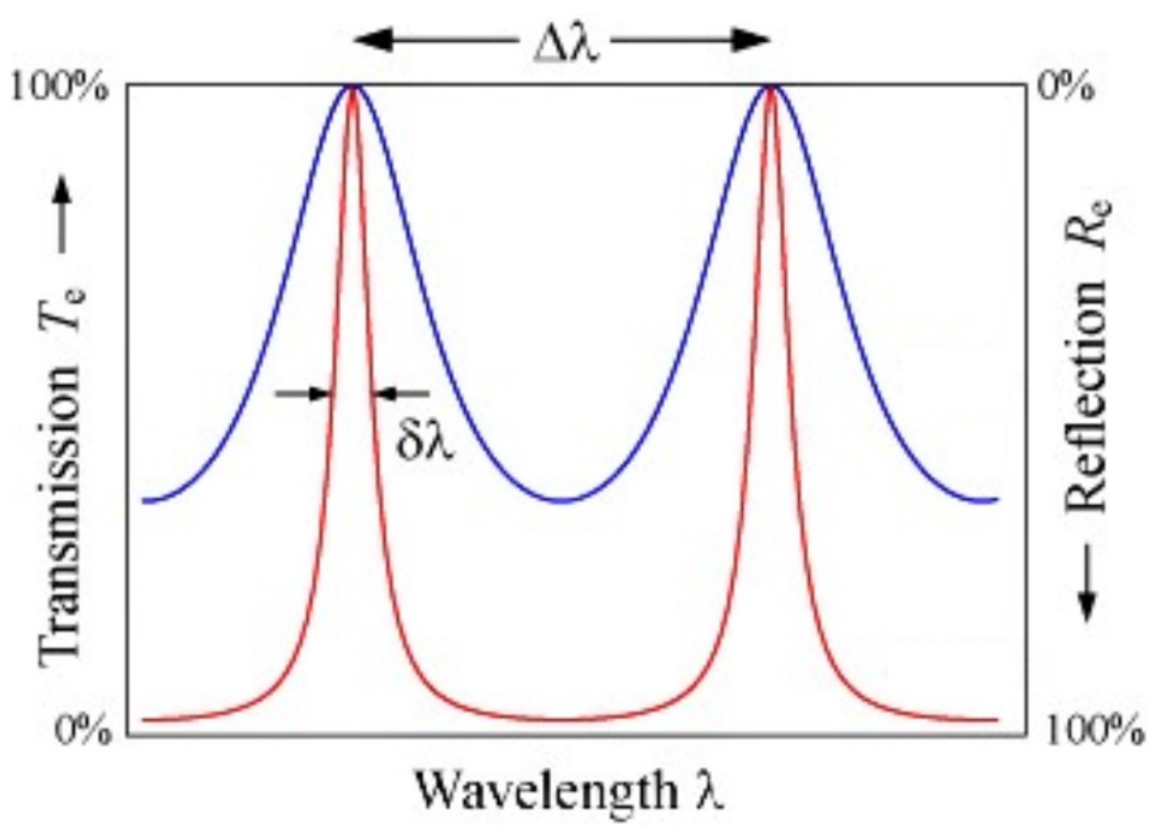

Figure 2.2 The resonance spectrum of a Fabry-Pérot Resonator.

\subsubsection{Photonic Crystal Resonator}

PCs are periodic structures designed to affect photon flow in the same way that energy levels in semiconductor crystals affect electron flow. PCs are typically composed of high dielectric constant materials in one, two, and/or three-dimensions. If a defect perturbs the supporting bandgap structure's periodicity, states within the bandgap may be present and lead to defects for light localization. The defect region's mode volume $(V)$ is the smallest of all resonating structures (Figure 2.1-center). Also, PCs resonator exhibit a high $Q / V$ ratio [12].

\subsubsection{Whispering Gallery Mode}

Lord Rayleigh was the first to discover the whispering gallery resonance phenomenon in the dome of St. Paul's cathedral in London [13]. He observed that sound 
could be heard at specific points along the dome's circumference, indicating that the sound waves form so-called WGMs within the dome structure. The same concept of sound reflection along the dome applies in the optical domain for micro-optic resonators of similar geometries [14].

In 1969, the acoustic concept was theoretically extended by Marcatili at Bell Labs into the optics regime [14]. He presented WGM in a micro-optic ring resonator, which has a higher refractive index than the surrounding. The optical field inside the resonator exhibited strong resonance behavior when the round-trip meets at the same point and has a phase shift equal to an integer multiple of $2 \pi$. Hence, the fields constructively interfere. These resonances significantly depend on the cavity's geometry, and the resonant modes are confined at the air-dielectric interface (Figure 2.1-right). The mathematical relationship for resonance is:

$$
\lambda_{r}=\frac{2 \pi n R}{m}
$$

where $n$ is the internal refractive index, $m$ is the integer multiple related to the mode order, $R$ is the radius of the structure, and $\lambda_{r}$ is the free space wavelength of the propagating light.

\subsection{Properties of an Optical Resonator}

An overview of the main properties of optical resonator cavities are presented, including Quality factor $(Q), F S R$, Finesse $(F)$, and Mode Volume $(V)$. 


\subsubsection{Quality Factor}

Resonance is characterized by a parameter called the Quality factor $(Q)$. The $Q$ factor indicates how lossy a resonator state can be. It is a characteristic of a resonator, defined as the ratio of stored energy to the power loss:

$$
Q=\frac{\left.\omega_{o} \text { (energy stored in the resonator }\right)}{\text { time-averaged power loss }}
$$

The $Q$ factor may also be expressed using the photon lifetime, $\tau$, and the oscillating optical field's frequency, $\omega_{o}=\frac{2 \pi c}{\lambda}$, as:

$$
Q=\tau \omega_{o}
$$

The $Q$ factor may also be determined from the frequency spectrum obtained by the Fourier transform of the field by finding the resonance frequencies of the signal and measuring the full-width-half-maxima (FWHM) of the resonant peaks (Figure 2.3). It can be expressed as:

$$
Q=\frac{\omega_{o}}{\Delta \omega}=\frac{\lambda_{o}}{\Delta \lambda}
$$

where $\omega, \lambda, \Delta \lambda$, and $\Delta \omega$ are the resonant frequency, wavelength, and FWHM of resonance Lorentzian shape in the wavelength and frequency domain, respectively. 


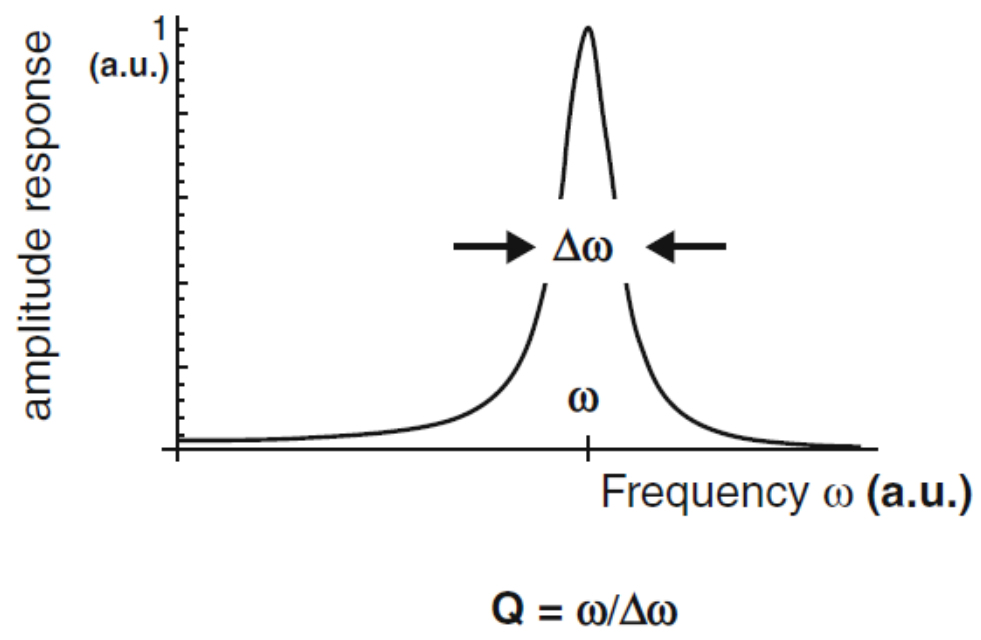

Figure 2.3 The resonator's Quality factor in the frequency domain.

The intrinsic $Q$ factor, $Q_{\text {int }}$, is always the sum of all losses in the resonator expressible as:

$$
\frac{1}{Q_{\text {int }}}=\frac{1}{Q_{\text {mat }}}+\frac{1}{Q_{s c t}}+\frac{1}{Q_{\text {surf }}}+\frac{1}{Q_{\text {rad }}}
$$

where $Q_{\text {mat }}$ is intrinsic material absorption, $Q_{s c t}$ is scattering losses from the surface roughness, $Q_{\text {surf }}$ is surface absorption loss caused due to surface coatings or contaminations, and $Q_{\text {rad }}$ is the bending loss or radiation loss arising from the total internal reflections at a curved interface. When the resonator is coupled to an external mode, the overall $Q$ factor, $Q_{\text {total }}$, gains an additional contribution, $Q_{\text {ext }}$ due to losses from that external mode.

$$
\frac{1}{Q_{\text {total }}}=\frac{1}{Q_{\text {int }}}+\frac{1}{Q_{\text {ext }}}
$$




\subsubsection{Mode Volume}

Another important property of the resonator is the mode volume, $V$. The mode volume is the effective volume the mode occupies if the energy density is homogeneous and equal to the original mode's maximum energy density. It is the confinement of light in the cavity expressible as:

$$
V=\int \frac{\varepsilon(r)|E(r)|^{2}}{\max \left(\varepsilon(r)|E(r)|^{2}\right)} d r^{3}
$$

The smaller the mode volume, the more tightly the mode is confined.

\subsubsection{Free Spectral Range}

The FSR is a normalized resonator property related to the resonant peaks' spacing to cavity length. It can be expressed as:

$$
F S R=\frac{\lambda^{2}}{2 l}
$$

where $l$ is the resonator's length. The FSR and length are inversely proportional to one another.

\subsubsection{Finesse}

Finesse is defined as the ratio of FSR and the FWHM linewidth of a resonance [15]. The resonator's finesse tells us the distance of the resonant peaks in terms of the number of FWHM linewidths between two consecutive resonant peaks. Simply, it can be explained as the number of bounces a light makes before leaking out or being absorbed. It is also 
equal to the $Q$ factor times the fraction of the spectrum covered by one FSR of the resonator [17].

$$
F=\frac{\Delta_{\omega_{F S R}}}{\Delta_{\omega_{F W H M}}}=Q \frac{\Delta_{\omega_{F S R}}}{\omega_{0}}
$$

\subsection{Cavity Structures}

Resonant structures are made from dielectric materials and metals [16, 17]. There are several various micro-optic resonator cavities whose operating principles closely resemble WGM. They are classified by their geometrical shape, see Figure 2.4.

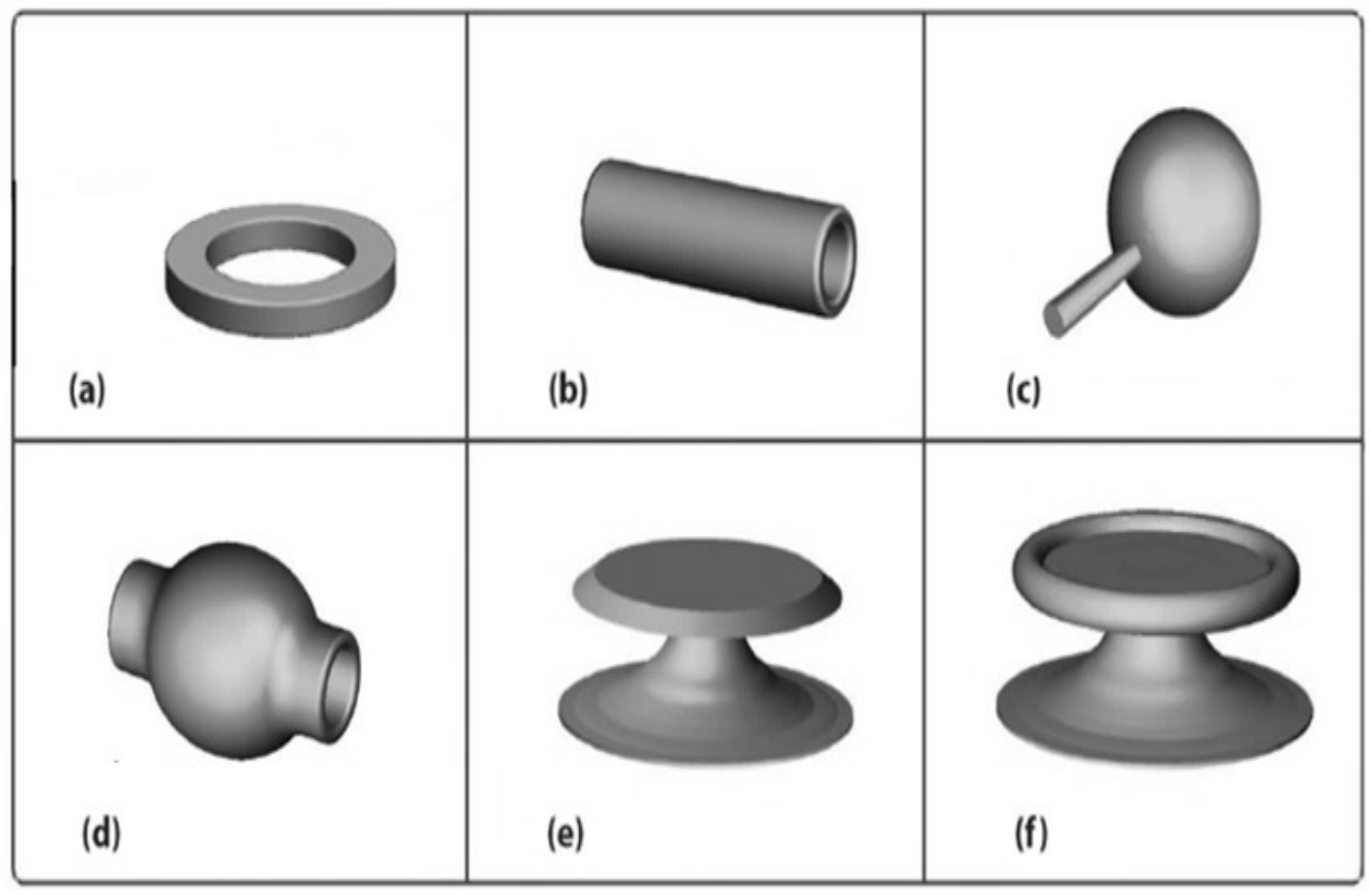

Figure 2.4 Geometrical representation of commonly used micro-cavity/resonator structures based on the WGM principle (a) micro-ring, (b) micro-capillary, (c) micro-sphere, (d) micro-bottle, (e) micro-disk, and (f) micro-toroid. 


\subsubsection{Micro Ring}

The micro ring resonator is a circular closed-loop waveguide, Figure 2.4(a). It can be fabricated using different materials such as silicon, silicon dioxide, and polymers. SOI waveguides channel light into the silicon core surrounded by a silicon oxide bottom cladding and a low index top cladding (oxide or air). A bus or other ring waveguide can be coupled to this resonator to transmit input and output signals. An array of these resonators can easily be fabricated, permitting wavelength division multiplexing. The $Q$ factor can be in the range of $10^{3}-10^{6}$. A new structure, the PANDA ring resonator, has been specifically designed to exploit the material's nonlinear optical properties. It has a center ring and two nonlinear side rings made of InGaAs [18].

\subsubsection{Micro Capillary}

The micro capillary is a hollow-core cylinder, Figure $2.4(\mathrm{~b})$. It can be made by stretching glass tubes. They are easily fabricated resonators with the ability to couple light in and out. The light is confined along its perimeter. This kind of resonator provides optical access to the analytes that flow within the hollowed core. The sensitivity measured from the micro capillary resonator depends on the radius and wall thickness. A thin-walled resonator overlaps the WGM fields with the analyte to provide improved sensitivity [19].

\subsubsection{Micro Sphere}

A micro sphere resonator is a 3-D structure. It resembles the shape of a ball or continuous round surface, Figure 2.4(c). The sizes of sphere resonators vary from $40 \mu \mathrm{m}$ to $400 \mu \mathrm{m}$, are easily fabricated, and are typically made from silica optical fiber. The 
fiber's tip is melted using electric arc heating, where the surface tension causes it to evolve into a round surface. The sphere shape immediately solidifies as the heat source is removed. The light travels around the edges (polar axis) of the micro sphere resonator; if it returns to the same point with the same phase, it constructively interferes to form resonance. The optical mode is confined in the polar direction. This resonator can have a low optical loss and ultra-high sensitivity. Due to minimal reflection loss and deficient material absorption, this resonator can achieve $Q$ factors up to $10^{11}[20]$.

\subsubsection{Micro Bottle}

The MBR has a cylindrical symmetry whose radius smoothly increases along the axial direction to a few microns forming an enlarged central region, and then the radius smoothly decreases, Figure 2.4(d). The enlarged central region takes a parabolic shape. It can be fabricated from optical fibers and/or polymers. The elongated axial width provides broader space for the mode confinement in this direction than the micro sphere resonator. The decreasing radii prevent light from leaking out of the resonator. The $Q$ factor can reach up to $10^{9}$ [21]. The MBR will be further discussed in the upcoming chapters.

\subsubsection{Micro Disk}

The micro disk resonator is a flattened cylinder of a small height, suspended over a silicon pillar of a few microns in height above the substrate, see Figure 2.4(e). It is millimeter-sized and fabricated using high-purity crystals, $\mathrm{SoI}$ or $\mathrm{Si}_{3} \mathrm{~N}_{4}$. The micro disk geometry allows 3-D confinement of light in both the transverse and in-plane directions with respect to its orientation. In the transverse direction, it behaves as a planar waveguide. 
In the in-plane direction, light circulates the edges of the disk. The micro disk is similar to the micro sphere in terms of the modes supported. The $Q$ factor is in the range of $10^{4}-10^{5}$ [22].

\subsubsection{Micro Toroid}

The micro toroid is a flat 3-D structure, but its essential properties rely on two dimensions. Its geometry can be described as a disk resonator with toroidal (i.e., doughnut) edges suspended over a silicon pillar by a silica membrane, see Figure 2.4(f). It is fabricated on an oxide-coated Si wafer. The torus' small diameter allows the optical modes to be tightly confined in the micro toroid compared to the micro sphere. It acquires the advantages of both the micro ring and micro sphere resonators. The structure has a smaller mode volume than the micro sphere, whereas the $Q$ factor can be $10^{7}-10^{8}$ [23].

\subsection{Sensing Principle}

Optical sensors are tools for qualitative and quantitative information acquisition of the analyte present in the resonator's surrounding environment. Any perturbation can cause the resonance's frequency to shift and affect the resonator's physical parameters (such as the size and refractive index). These perturbations can be a bulk change in refractive index, a single nanoparticle, a small number of compounds, temperature, pressure, or stress-strain. Each of them corresponds to a different kind of change in the transmission spectrum [2425]. Sensing modalities are techniques used to extract these desired changes caused by perturbations. 
The advantages of optical sensing are that it does not alter the materialistic properties of the resonator. The resonator's interaction with the particles (bulk, single, or molecular compounds) present in the surrounding medium can be controlled for improved detection response using various sensing modalities.

For an MBR to work as an optical chemical sensor, the optical beam will excite the MBR's WGM, which then interacts with the analyte (single nanoparticles or molecular compounds whose presence is to be detected), causing the resonance frequency to shift. The change in the resonance spectrum can be used for further sensitivity calculations. For an MBR optical temperature sensor, such as an optical fiber resonator configuration, a physical temperature change may modify the resonator's size and refractive index, resulting in a measurable wavelength shift.

Some of the commonly used sensing modalities are discussed below:

\subsubsection{Mode Shift/Reactive Shift}

Mode shift, also known as reactive shift, is one of the most common sensing modalities. Any small perturbation in the refractive index, temperature, and/or resonator's shape will result in a slight change in the resonant wavelength (or frequency), see Figure 2.5. The temperature changes introduce two other effects onto the resonator: the thermooptic effect (changes the refractive index) and linear thermal expansion (changes the resonator's size); more details are in Section 4.2.1. It is used to detect various chemical analyte types, such as bulk refractive index changes, monolayer adsorption, and singlemolecule perturbations. Refractometers, for example, directly aim to monitor changes in 
the bulk refractive index [26]. The author's work for gas detection using MBR works on this mode shift modality [27].

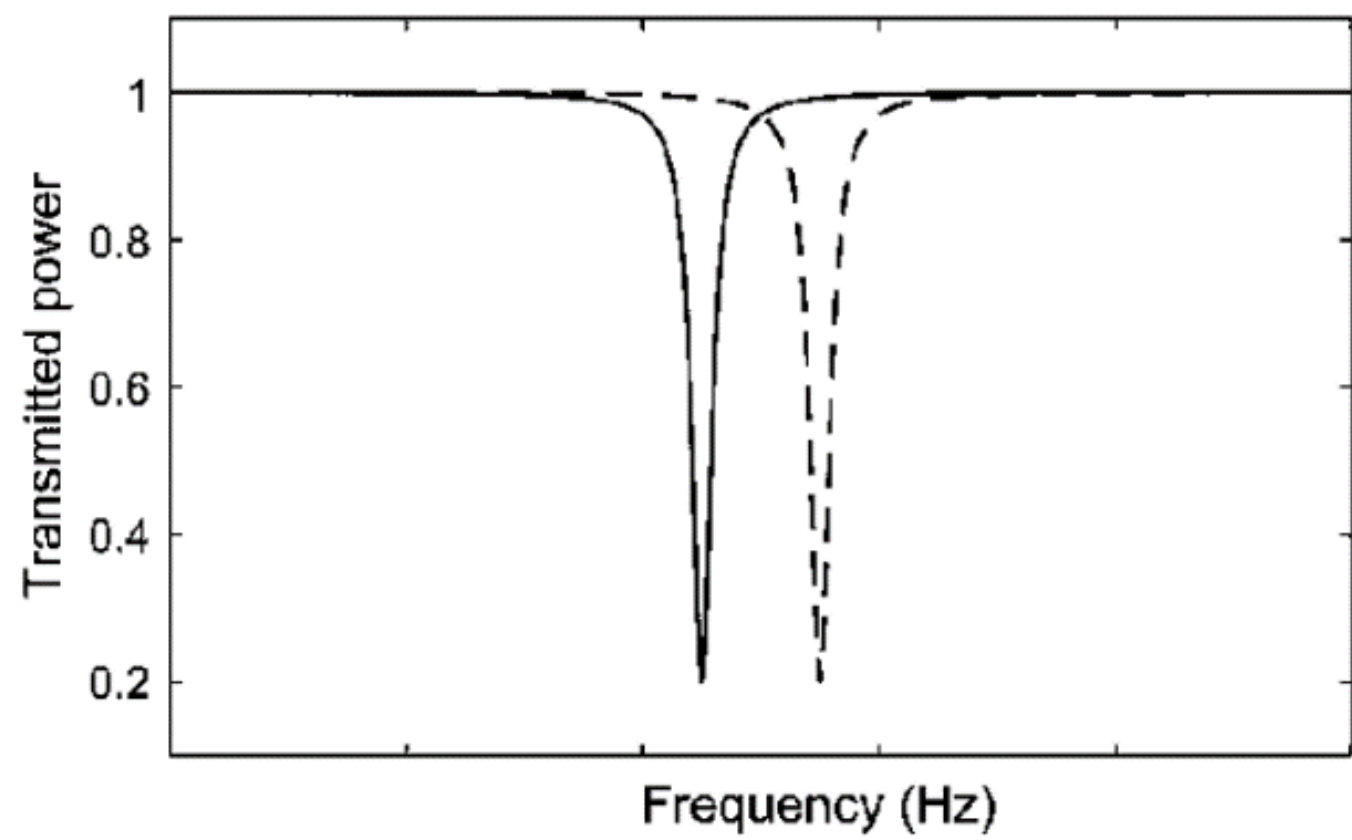

Figure 2.5 The dotted line is the original spectrum. The solid curve is the mode shift after the analyte is introduced in the resonator surrounding.

\subsubsection{Mode Splitting}

Typically, a resonator possesses two degenerate WGMs with the same resonant frequency/wavelength and field distributions but in clockwise $(\mathrm{CW})$ and counter clockwise (CCW) propagation directions. With the introduction of a scatterer (direction-specific perturbation), surface roughness, or material inhomogeneity, the resonator deviates from its perfect azimuthal symmetry in the mode volume. There will be backscattering inside the resonator, which couples to the $\mathrm{CW}$ and $\mathrm{CCW}$ modes. Because of this coupling, degeneracy between the two modes may be lifted [28-30]. The mode's resonance splits into a doublet (Figure 2.6). 
This mode splitting technique is suitable for detecting the number of nanoparticles using scatterer-induced coupling between the $\mathrm{CW}$ and $\mathrm{CCW}$ propagating modes. This technique's drawback is that it is insensitive to the constant change in the bulk refractive index in the resonator's ambient medium. If, for instance, a uniform layer is deposited on the resonator's surface, no mode splitting will occur-[31-32].

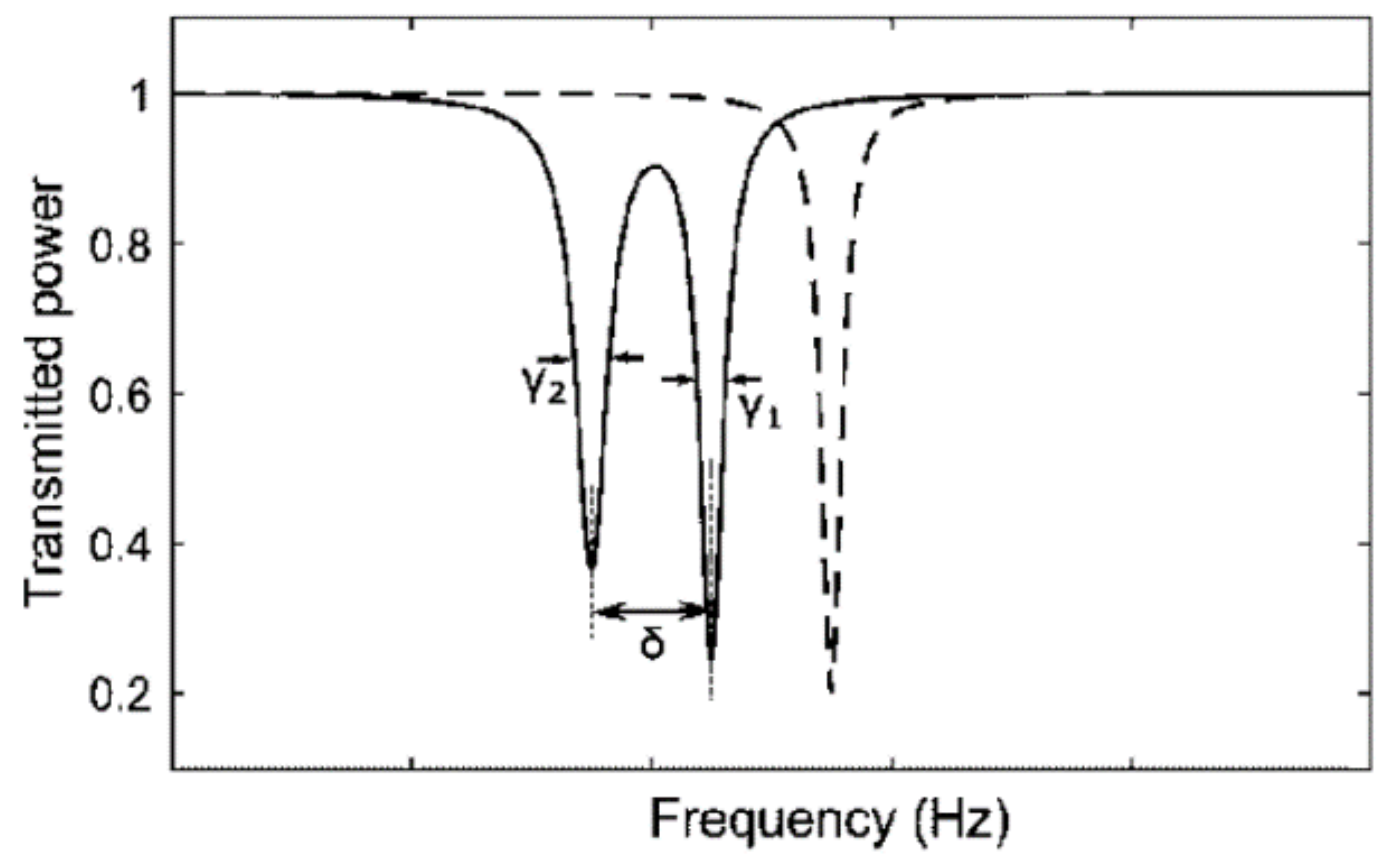

Figure 2.6 Mode splitting sensing modality: the dotted line is the original spectrum, and the solid curve represents the mode splitting after the analyte is introduced onto the resonator surface.

\subsubsection{Mode Broadening}

Mode broadening widens the linewidth with the shift in the resonator's frequency or wavelength, shown in Figure 2.7. A single nanoparticle is attached to the resonator; it breaks the rotational symmetry scattering light between $\mathrm{CW}$ and $\mathrm{CCW}$ modes and possibly other modes. Scattering into $\mathrm{CW}$ and $\mathrm{CCW}$ modes gives rise to non-degenerate coherent 
superposition, yielding two new states in the form of standing waves. One will have a maximum field, and the other will have a minimum field at the particle position. This scattering into modes will lower the $Q$ factor and introduce broadening of the standing wave WGMs [33].

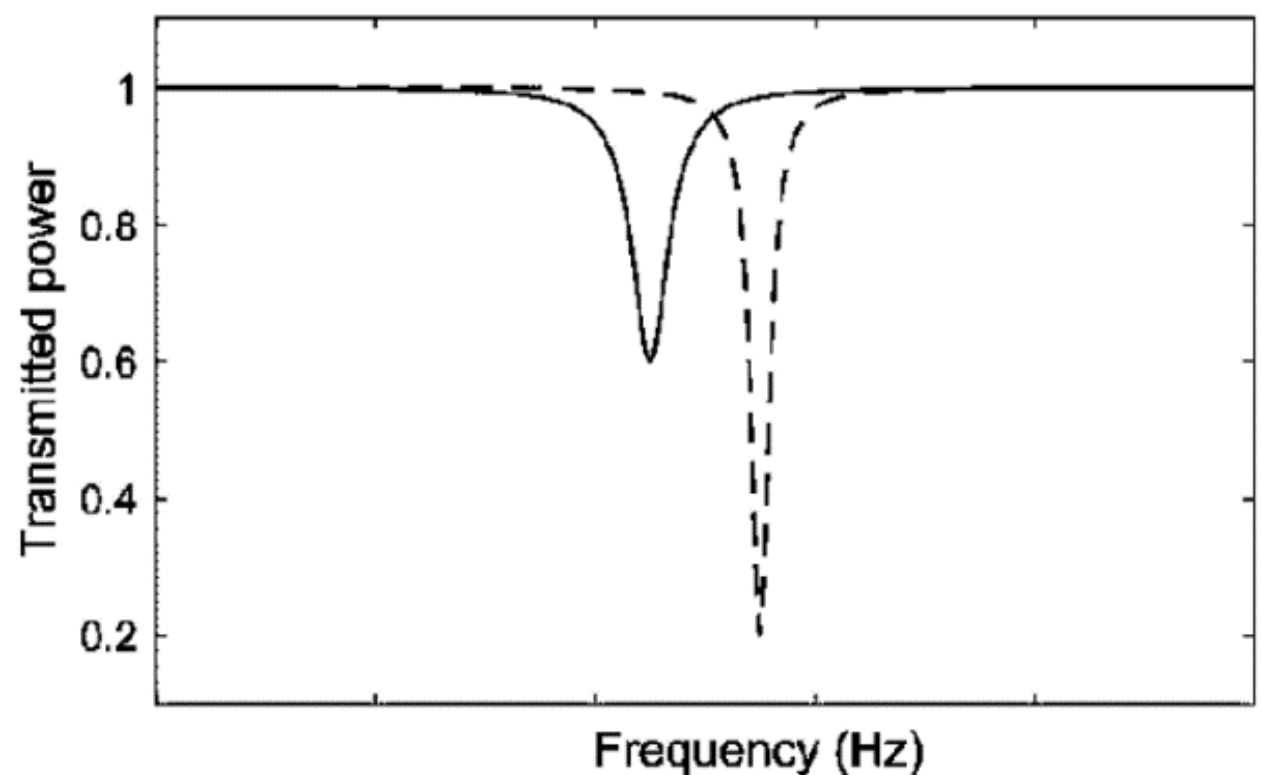

Figure 2.7 In the mode broadening sensing modality, the dotted line is the original spectrum. The solid curve shows the broadened mode when the analyte is placed on to the resonator's surface.

Broadening caused by every single nanoparticle is distinct, mainly due to its position on the resonator shown in Figure 2.8. As the number of particles increases, the linewidth increases. The detection from a reactive shift and mode splitting is susceptible to external noise. To overcome noise issues, one must create a microcavity with an ultra-high $Q$ factor. In contrast, mode broadening is immune to noise from the laser and environmental disturbances. A deformed, polydimethylsiloxane (PDMS)-coated cavity can significantly 
suppress the thermo-optic noise induced from the laser and environmental thermal drift [34]. Besides, the microcavity does not need to possess an ultra-high $Q$ factor [35]. It was also observed that coupling strength does not affect the linewidth resonance dips [36]. Linewidth measurements are self-referenced and external drifts do not reduce their accuracy [37]. Broadening provides a better detection limit [35].

To conclude, sensing mechanisms for particles smaller than the WGM wavelength give rise to reactive shift and mode splitting, whereas, for a single nano-sized particle, the mode broadening is the dominant effect.

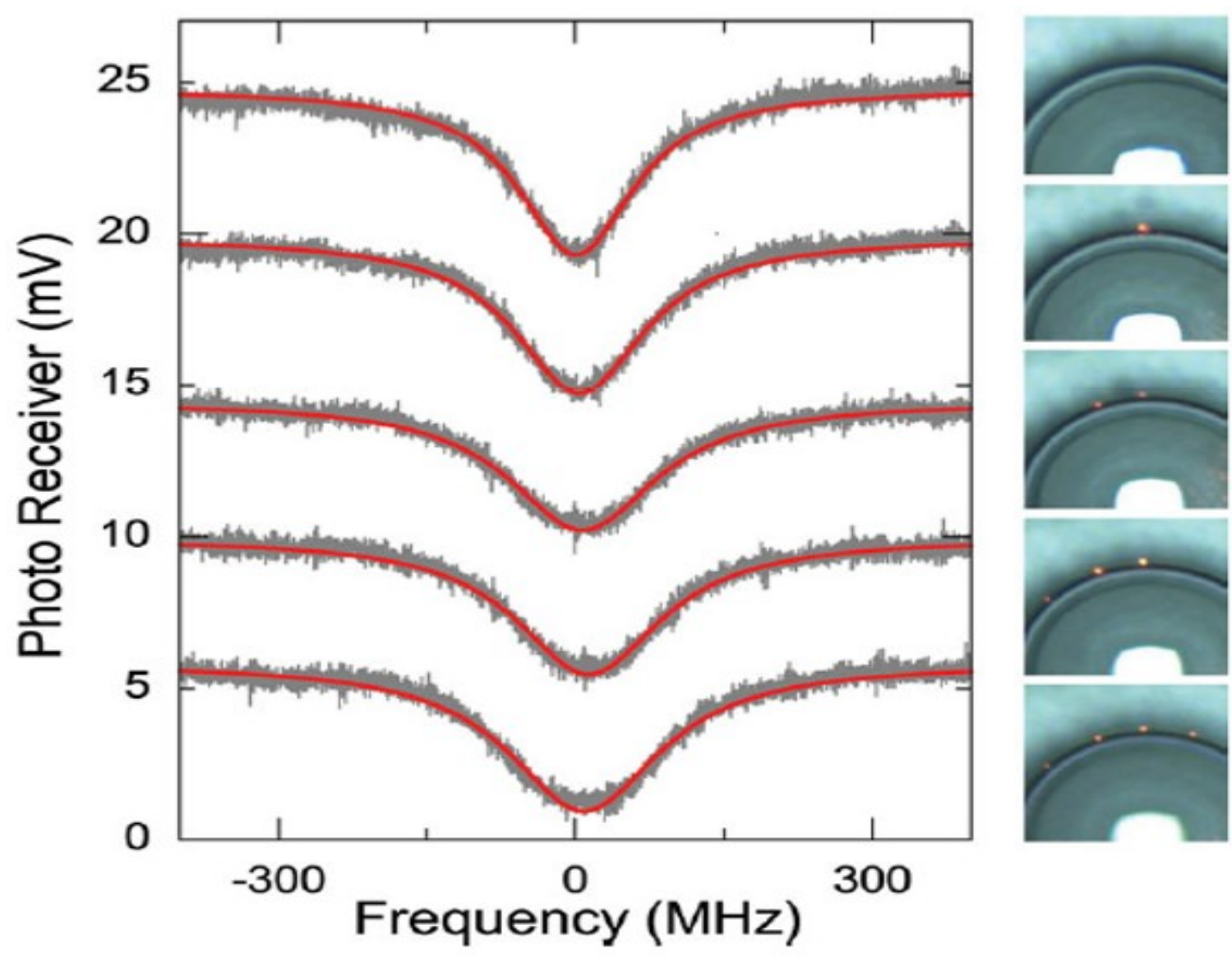

Figure 2.8 Transmission spectrum of 0 to 4 nanoparticles attached to the surface excited by a visible light laser. The figure is reproduced with permission from [38]. 
In this chapter, the WGM resonance condition is discussed in detail. The sensing modalities, characteristics, and various optical cavities based on the same concept are also presented. Among these structural profiles, the optical MBR was found to be the least explored. Its unique features motivated the author to further research in this particular field of micro-optic resonators. From this point onwards, the main focus of discussion will be on MBR and its various applications. 


\section{Chapter 3: Literature Review on the Micro-optic Bottle Resonator}

In this chapter, the discussion focuses on the MBR. As previously indicated, the MBR generally has a cylindrical symmetry with an enlarged central region, from $20 \mu m$ to $500 \mu \mathrm{m}$, bounded by smoothly decreasing radii in both axial directions. The MBR can also display a high $Q$ factor on the order of $\left(10^{9}\right)$. The numerical computation and potential application orientation of the MBR geometry are examined in this thesis for significantly reduced structure from $1 \mu m$ to $5 \mu m$ range. The intention was to perform proof of design using a scaled-up version of the MBR fabricated at Carleton and Royal Military College (RMC) in Kingston. Such effort was cut short due to limited lab access imposed by the global pandemic.

In 2004, the pioneer, M. Sumetsky, introduced a micro-optic resonator that looked like an elongated spheroid and named it a Whispering Gallery Bottle [21]. Its geometry shows 3-D WGM confinement, as shown in Figure 3.1. The most common MBR configuration consists of an axially enlarged region on a solid [39] or hollow glass fiber [40]. It is a cylindrical WGM resonator whose radius increases from both ends smoothly up along the axial direction, forming a ridge. The ends of the radii then smoothly taper down, making the overall structure resemble a double-neck bottle, preventing light from leaking out of the bottle. The enlarged region's radial and axial profiles determine the modes' optical properties that may circulate in the azimuthal direction. It also provides flexibility to excite bottle-modes and selectively exhibits an ultra-high $Q\left(10^{9}\right)$. If the 
coupling is moved, irrespective of its position between the two ring indentations, WGMs may be excited [41].

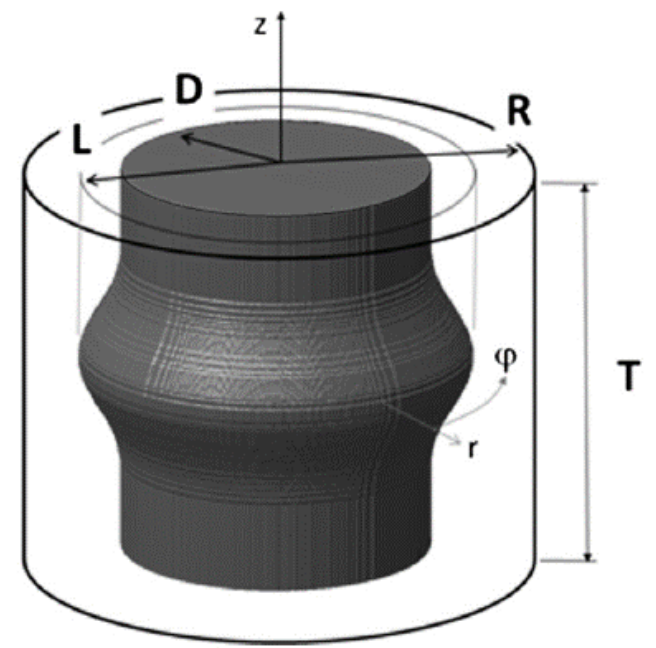

Figure 3.1 Schematic profile of the Micro-optic Bottle Resonator.

\subsection{Fabrication}

Most MBRs are fabricated starting with uniform optical glass fiber. This fabrication method, as well as other more elaborate approaches, are discussed below.

\subsubsection{Melting Optical Glass Fiber by a $\mathrm{CO}_{2}$ Laser}

Heating fiber through a laser source is different from heating with a flame or fusion splicer. Heat is internally-generated by optical absorption within the fiber as opposed to thermal conduction from an external source. In this fabrication method, the two tapered ends of the MBR are produced by melting the glass with the laser light and pulling on the softened glass, reducing the axial radii. A smooth ridge (bottle region) is formed between the two tapers, as shown in Figure 3.2 [42]. In collaboration with the RMC, an attempt was made to fabricate the MBR using the laser heating method. 


\subsubsection{Softening and Compression}

In this process, the ends of two glass optical fibers were cleaved flat. Both ends were axially aligned and pushed towards each other while continuous heat was applied using an arc discharge. At the melting point, the ends soften and fuse, and through compression, generate an enlarged region, as shown in Figure 3.2. Multiple arcs and control over the compression pressure are typically used to shape the MBR [43].

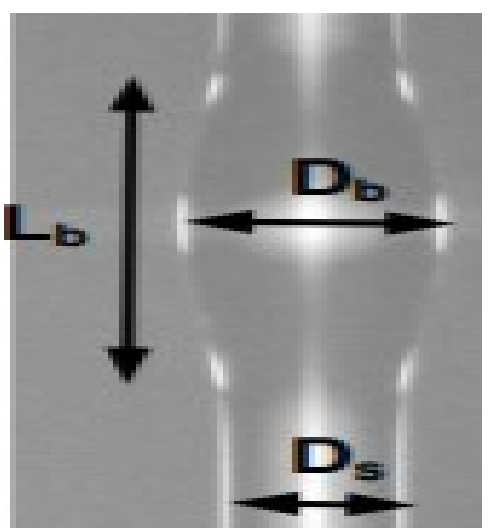

Figure 3.2 MBR fabrication either by $\mathrm{CO}_{2}$ laser or by a softening-compression method. Here, $L_{b}$ is the axial width, $D_{b}$ is the bottle diameter, and $D_{s}$ is the tapered end's diameter.

\subsubsection{Rolled Up Silicon Layer}

The self-rolling mechanism of the strained semiconductor bilayer fabricates 3-D objects from a 2-D epitaxial layer. The fabrication steps follow by growing a buffer layer on top of the GaAs substrate. The $40 \mathrm{~nm}$ AlAs serve as a sacrificial layer in the lift-off process. The $20 \mathrm{~nm}$ epitaxial growth of the $\operatorname{In}_{0.2} \mathrm{Ga}_{0.8} \mathrm{As}$ together with the $30 \mathrm{~nm}$ of GaAs forms a strained bilayer. The U-shape is grown by shallow wet etching using $\mathrm{H}_{2} \mathrm{O}_{2} / \mathrm{H}_{3} \mathrm{PO}_{4}$ solution into the $\operatorname{In}_{0.2} \mathrm{Ga}_{0.8} \mathrm{As}$. The edges are defined by deep etching through the sacrificial layer. The last step is highly selective HF etching to undercut the strained layer. Now the 
self-rolling mechanism starts, and multiwalled tubes are formed. As one side exhibits a Ushape, the other continues to roll up. The central tube is raised to a self-supporting bridge, and the middle part is separated from the substrate [44], Figure 3.3.
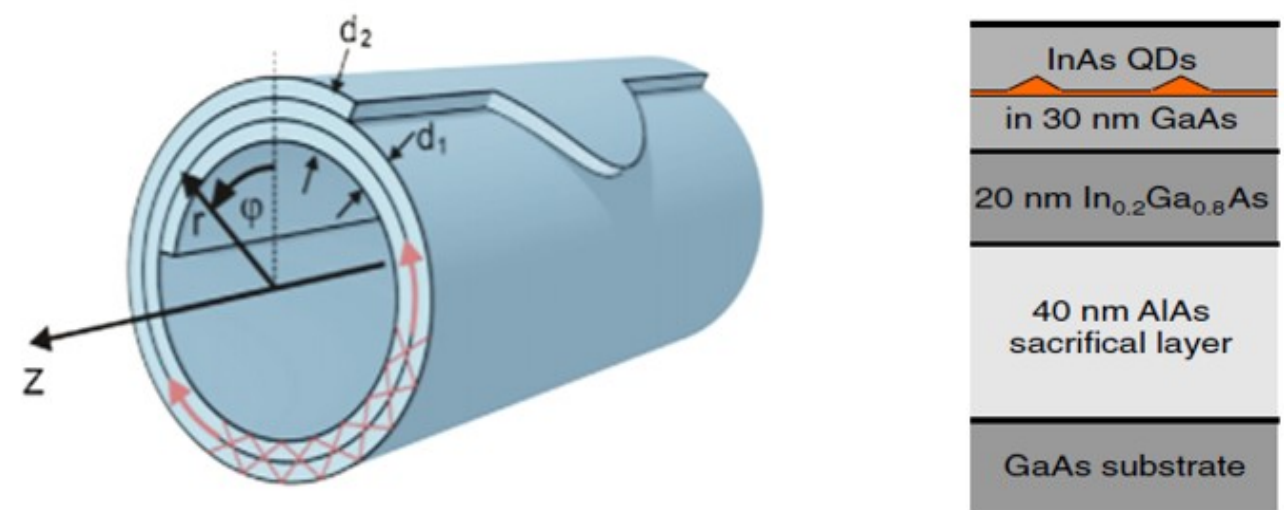

Figure 3.3 The bottle resonator's schematic exhibiting a parabolic lobe on the outer rolling edge is on the left. A schematic of the sample fabrication is on the right.

\subsubsection{Micro Machining}

The MBR is micromachined using a mask-less direct laser writing photolithographic system known as SF-100. A negative photoresist SU-8-2 is deposited on the glass fiber. The sample is prebaked and undergoes UV light exposure where the ring indentations are formed around the fiber, and the spacing between the rings is the desired bottle region. Once the pattern is transferred to the sample, it is post-baked to a specific temperature. It is then developed for a few seconds, and the last step is to hard-bake at 200 ${ }^{\circ} \mathrm{C}$. This thesis uses the micromachining process for MBR designing on the fiber, as shown in Figure 3.4. The details related to this process are discussed in Appendix A. 


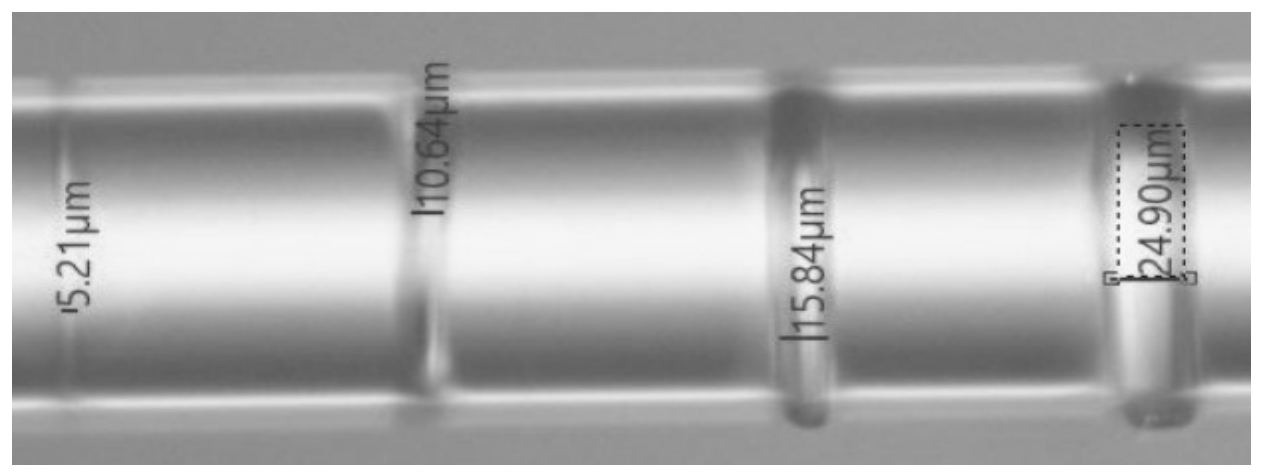

Figure 3.4 The MBR is micromachined by a mask-less laser writing machine on the fiber using a photolithographic process.

\subsection{MBR Excitation}

The excitation of the MBR's WGM is generally performed through evanescent wave coupling. Evanescent waves occur at the air-high dielectric medium boundary when the externally incident light's angle is greater than or equal to the critical angle. An evanescent wave exists parallel to the glass-air interface, with the field's amplitude exponentially decreasing with penetration depth into the air-high dielectric medium. The coupler is placed in close contact with the MBR. There, two evanescent fields exist, one decaying (from WGM) and the other growing (from the coupler). These two fields will superimpose, a phase-matching condition will be satisfied, and a WGM is excited. Once the fiber sample is ready to be tested, there are several ways to launch light into it [24] discussed below.

\subsubsection{Tapered-fiber Coupling}

Coupling bottle-shaped fiber to another tapered-fiber carrying a light is shown in Figure 3.5. It is graphically illustrated that the laser source can be used to pump light into 
the tapered-fiber (shown in green color). The tapered-fiber is coupled to the MBR (shown in red color) for WGM resonance excitation. The excited resonance will then travel through the tapered-fiber (shown in orange color). These mechanically tapered fibers are very fragile, and their surface is prone to contamination. However, this coupling can provide a $99 \%$ efficiency [45].

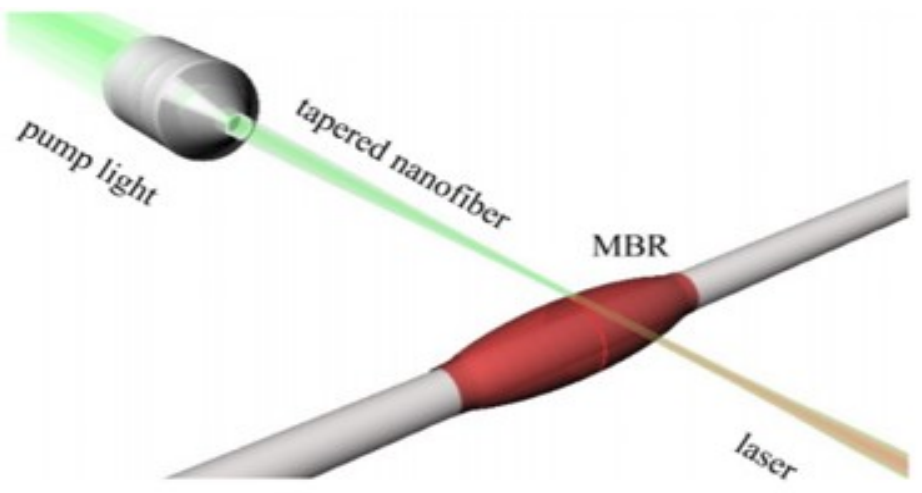

Figure 3.5 The MBR is coupled to tapered-fiber. Reproduced with permission from [45]

\subsubsection{Prism Coupling}

Prism coupling is one of the easiest methods to couple light within the MBR. The prism is placed on top of the bottled region sufficiently close enough, as shown in Figure 3.6. When the light is shined into the prism, they travel through evanescent fields and superimpose into the MBR's WGM resonance for mode excitation. When optimized, 80\% coupling efficiency can be achieved [46].

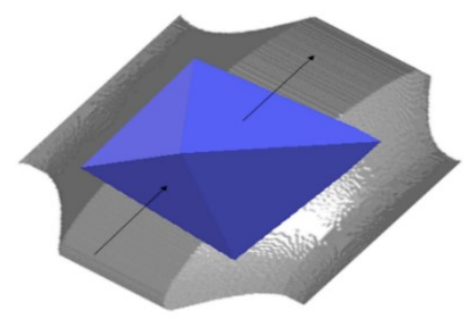

Figure 3.6 MBR coupled to the prism. 


\subsubsection{Angled Polished Fiber Coupling}

The end face of an optical fiber is angle polished and placed in close contact with the MBR, as shown in Figure 3.7. The light is launched into this fiber coupler, and an evanescent field superimposes the MBR's WGM for mode excitation. The efficiency is decreased to approximately $60 \%$ [47].

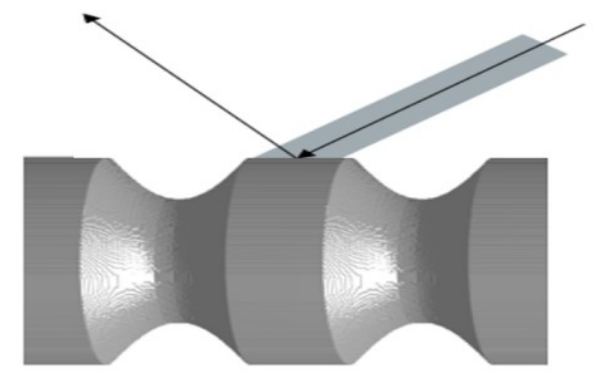

Figure 3.7 MBR coupled to an angled-end (polished) fiber.

\subsection{MBR Applications}

The MBR finds itself a candidate as the base structure of numerous practical applications. The most often encountered are the laser resonator, delay line, quantum electrodynamical devices, nonlinear, optomechanical structures, and sensors. Each of these are examined below.

\subsubsection{Laser Resonator}

A laser can be formed out of MBR, using its mode selectivity characteristic. The WGMs of MBR are spatially separated along the axial width direction. If one mode is excited at a time, it could be utilized in resonator lasing. One method of selective mode excitation is to spatially engineer the input pump of the laser [48]. When the laser's light is uniformly pumped, all modes are excited, and the MBR exhibits multimode behavior 
(Figure 3.8 (a)). If a small cavity size of less than $6 \mu \mathrm{m}$ is used, the larger FSR allows few axial modes within the gain curve. By engineering the laser intensity (dividing into multiple beams), only one beam will produce an interference distribution on the resonator's surface, as seen in Figure 3.8 (b). The beam's axial position can spatially overlap with the desired WGM, and only the excited WGM can lase; the rest of the modes are suppressed.

In this thesis, the FFB numerical technique can compute the single-mode lasing property, as mentioned in this application. The excited 3-D WGM can be displayed circulating the bottle resonator, as shown in Figure 3.8(b).

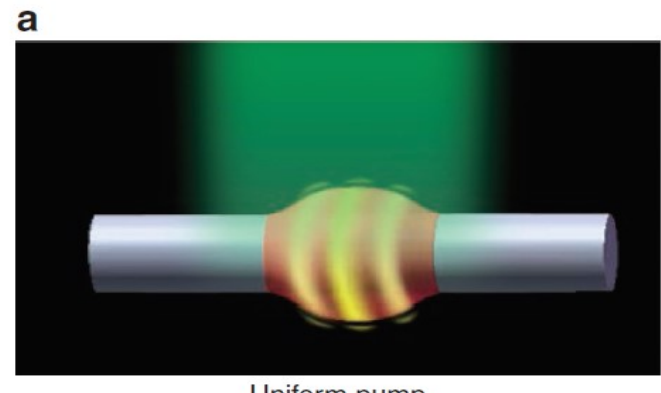

Uniform pump

b

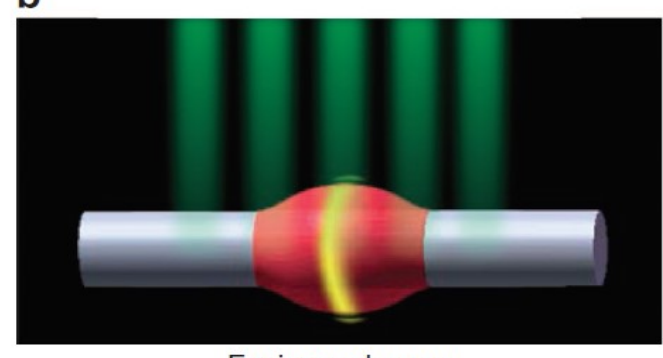

Engineered pump
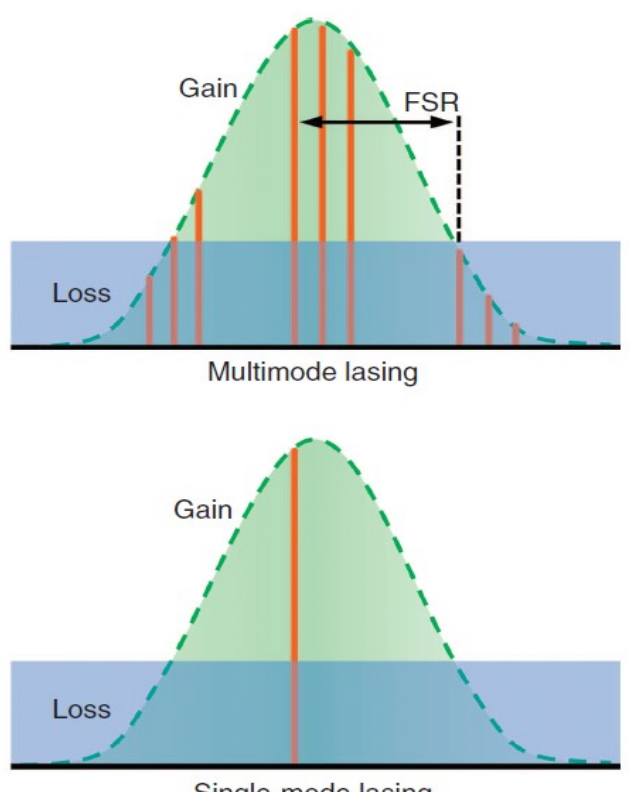

Single-mode lasing

Figure 3.8 The principle of single-mode WGM lasing in a polymer micro-resonator. (a) shows the multimode lasing behaviour and (b) shows the engineered pump intensity can modify the spatial gain profile of bottle WGM, enabling single-mode lasing. This figure has been reproduced with permission [48]. 


\subsubsection{Delay Lines}

The MBR delay line is an alternative to the miniaturized optical delay line in silicon photonics [49]. It is a different type of delay line, not based on the modulation of the refractive index. The slow light delay line is engineered from coupled nanoscaled micro resonators. Slow light propagation along the MBR axis is ensured by its rotation along an ultralow loss optical fiber's surface. For example, the MBR with semi parabolic nanoscale radius variation can be phase-matched to the input-output microfiber to perform a delay of 100 ps telecommunication pulses by several nanoseconds (Figure 3.9) [50].

This delay line MBR has a nanoscale parabolic radius variation coupled with an input-output micro tapered-fiber waveguide. These small-scale variations cause this resonator to perform a multi-nanosecond delay of light at telecommunication wavelengths when a light pulse launched from the input tapered waveguide into the MBR at contact point $\mathrm{Zc}_{\mathrm{c}}$ slowly propagates along the resonator axis in both directions and returns after reflecting from turning point $\mathrm{z}_{\mathrm{t} 1}$ and $\mathrm{z}_{\mathrm{t} 2}$ as shown in Figure 3.9 (a). After completing one round trip between one of the turning points and the contact point, few pulses are reflected and do not fully return into the tapered waveguide due to a phase mismatch between the tapered-fiber and MBR. These bouncing pulses between turning points have decreasing amplitude. The phase mismatch problem is solved by suppressing the transmission oscillations at the tapered-fiber end.

The FFB can be used to build the nanoscaled smooth variation along the bottle's radius, as shown in Figure 3.9 (b). Computations can be performed at telecom wavelengths. 
The excited WGM's variations while travelling along the bottle surface in the axial direction can be observed as the mode profile.

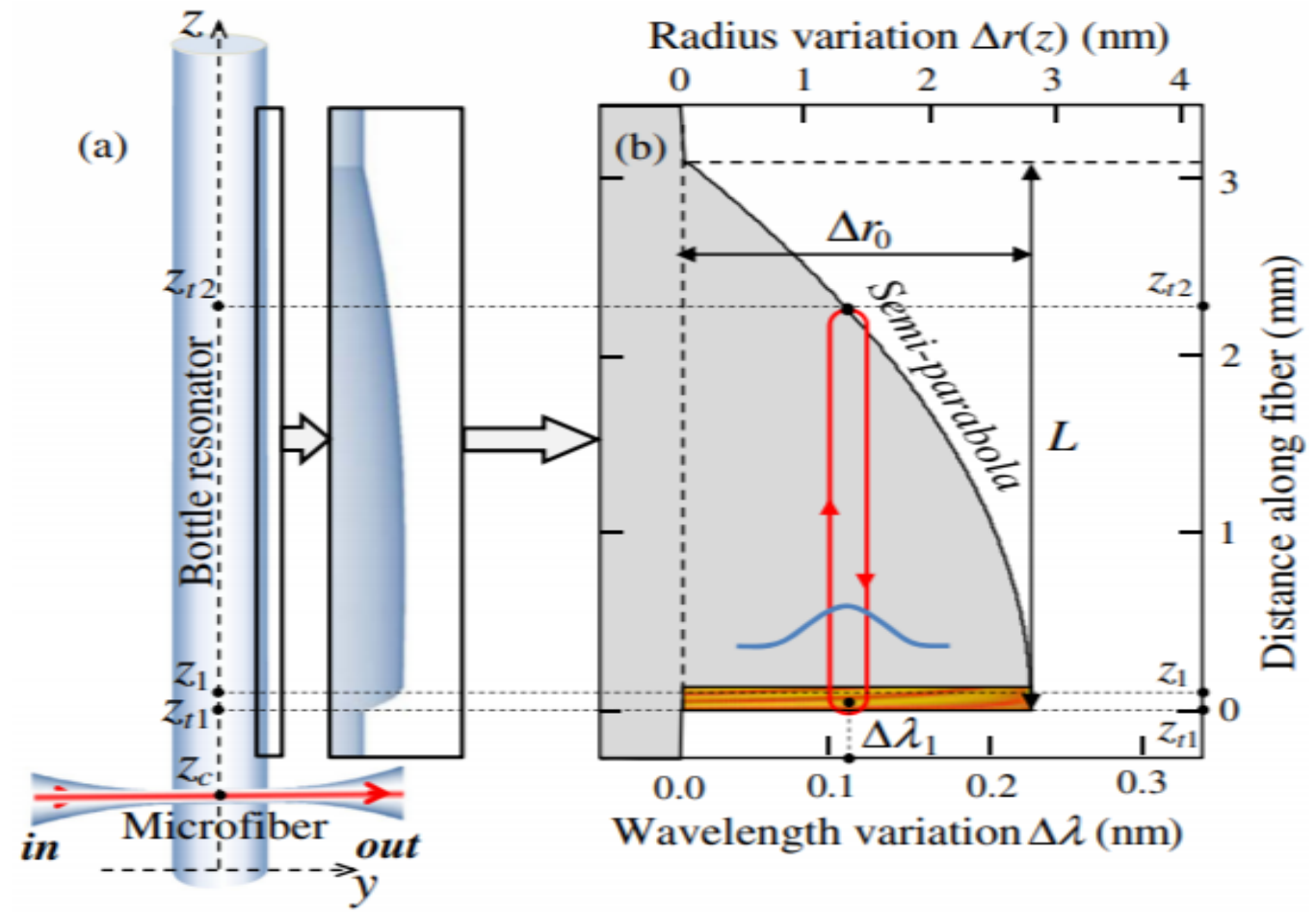

Figure 3.9 (a) Illustration of an optical bottle resonator delay line. Light is coupled into the resonator from a transverse waveguide (microfiber) and experiences WGM propagation along the resonator surface. The inset shows the magnified profile of the fiber radius variation. (b) Semi parabolic variation of a bottle resonator radius used in the numerical simulations. The figure has been reproduced with permission from [50].

\subsubsection{Quantum Electrodynamics}

Cavity quantum electrodynamics investigates quantum phenomena between particles, atoms, and photons, in an optical cavity [51]. MBR coupled to two input-output microfiber tapers demonstrates a fiber integrated quantum optical circulator, operated by a single $\mathrm{Rb}$ atom, as shown in Figure 3.10. 
The coupling coefficient $\kappa_{a}$ and $\kappa_{b}$ between the resonator field and field in the respective coupler "a" or "b" are adjusted so that both fibers are approximately and critically coupled to the empty resonator: $\kappa_{\mathrm{a}} \approx \kappa_{\mathrm{b}} \gg \kappa_{0}$, where $\kappa_{0}$ is the intrinsic resonator field decay rate, providing an efficient route for the atom. When no particle is coupled to the resonator mode, this realizes an add-drop filter in which light that is launched into one fiber will be transferred to the other fiber via the resonator. Because of its strong transverse confinement, the CW propagating resonator mode's evanescent field is almost entirely circularly polarized. Its electric field vector rotates $\mathrm{CCW}$ in the plane orthogonal to the resonator axis (z-axis), corresponding to $s$ - polarization. Time reversal symmetry then implies that the CCW propagating mode's evanescent field is almost entirely $s \pm$ polarized.

When the $\mathrm{Rb}$ atom is coupled to the resonator, light in $\mathrm{CCW}$ mode interacts strongly with the atom, whereas light in the CW mode exhibits much weaker coupling. The presence of a particle changes the resonator field decay rate. The add-drop functionality is maintained when light is launched into those fiber ports it couples to $\mathrm{CW}$ mode. For the other two input ports, the light couples to the $\mathrm{CCW}$ mode and the resonator atom system operates in the under-coupled regime. Thus, the incident light field remains in its initial fiber. The device is realized as an optical circulator that routes light from the input port to the adjacent output ports: the circular concept process and route classical signals at ultralow light levels in integrated optical circuits and networks.

The FFB can compute single-particle non-degenerate perturbations. The resonator structure's perturbation contributions can be isolated as a separate matrix that calculates the resonator state. 

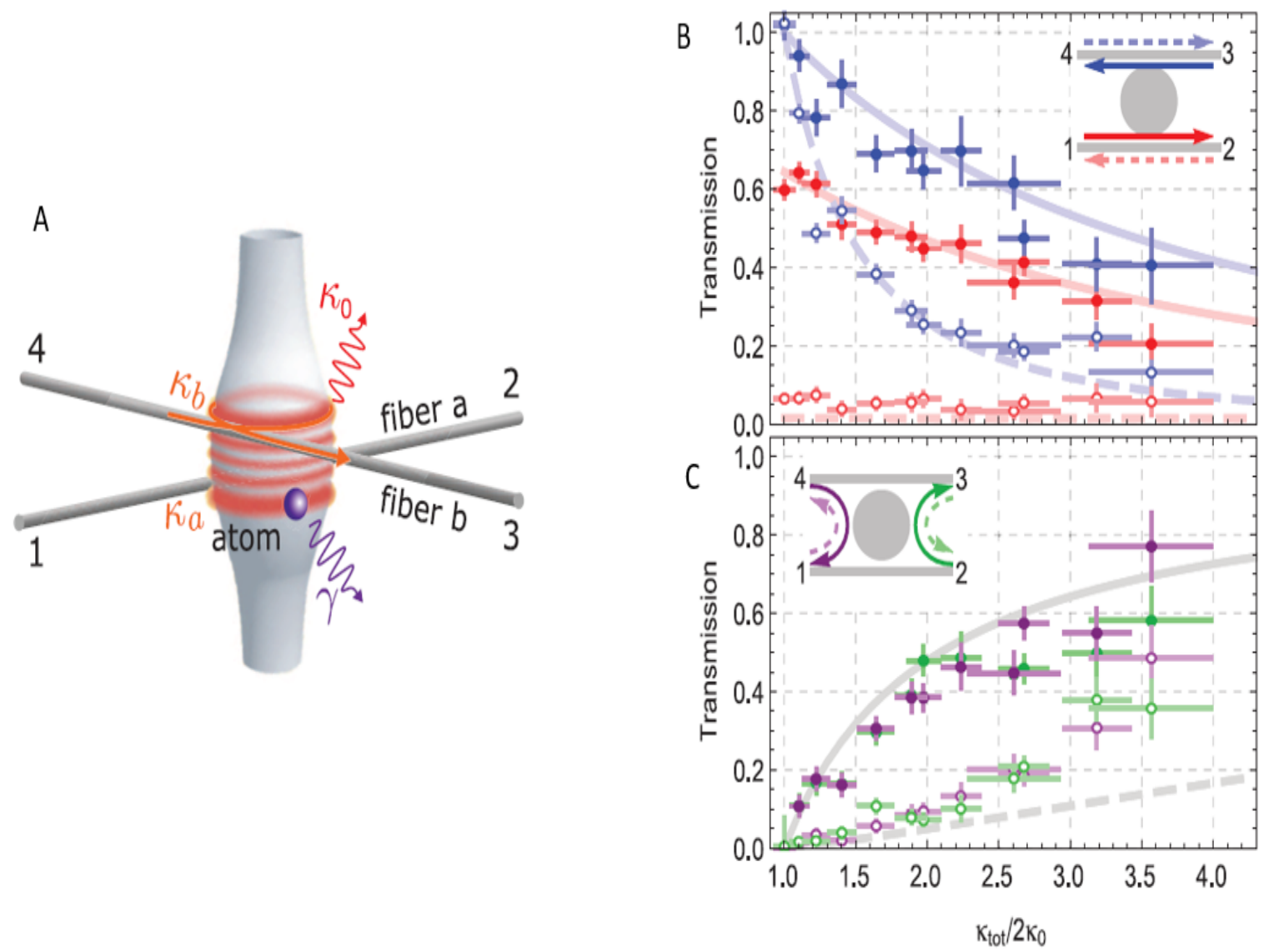

Figure 3.10(A) Schematic of MBR and single Rb atom coupled with two input-output microfibers. ( $B \& C)$ Port to port transmission vs normalized field decay of fiber coupled resonator. The solid circles indicate transmission in the forward direction and open circles indicate transmission in the backward direction. The figure is reproduced with permission from [51].

\subsubsection{Nonlinear MBR}

Nonlinear optics enhances the light-light interaction. When the material of the resonator exhibits third-order susceptibility, $\chi^{(3)}$ its refractive index $n$ depends on the intracavity intensity via the Kerr effect $n=n_{1}+n_{2} I$, where $n_{1}$ is the linear refractive index, $n_{2}=\operatorname{Re}\left(\chi^{(3)}\right)$ is the nonlinear refractive index, and $I$ is light intensity. Intracavity 
variation can modify the optical path length inside the resonator, and its transmission properties are changed [52]. This nonlinear effect is used in all-optical switching (controlling or redirecting light flow) via the Kerr effect when the resonator is operating as an add-drop configuration, see Figure 3.11.

The FFB can provide flexibility to define a structure's material properties such as the real part, extinction coefficient, and electric susceptibility. Changes in these properties will be observed as a wavelength shift.

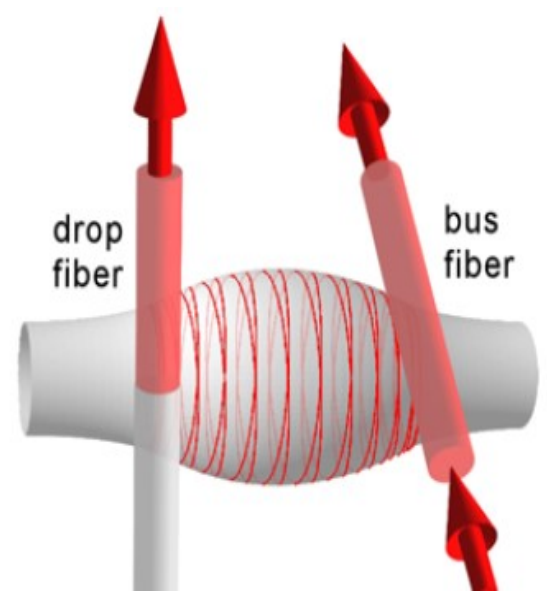

Figure 3.11 Schematic of an add-drop coupling configuration using two ultra-thin coupling

fibers. The spiraling red line in the resonator traces the ray path. This figure has been reproduced with permission from [52]

\subsubsection{Optomechanical MBR}

The photons propagate along the boundary of the resonator via TIR. The mechanism leading to the coupling of optical modes of frequency $\omega_{r}^{(o p t)}$ and mechanical modes of frequency $\omega^{(m e c h)}$ is momentum transfer from intracavity photons (via radiation pressure) to vibrational mechanical modes of the resonator. The coupling causes distortion and modification in the optical path length, which shifts the resonance frequency creating 
sidebands on the intracavity and are separated by multiple mechanical fields $\omega_{r}{ }^{(o p t)} \pm$ $b \omega^{(\text {mech })}$, where $b$ is a positive integer. The first pair of sidebands are located at $\omega_{r}{ }^{(o p t)}-$ $\omega^{(m e c h)}$ and $\omega_{r}^{(o p t)}+\omega^{(m e c h)}$ and are called Stokes and anti-Stokes, respectively [10]. Figure 3.12 depicts an illustration of MBR mechanical modes excited with a laser.

The mechanically induced stress-strain radiation pressure can increase the resonator's size, causing the refractive index to change can be easily computed using the FFB mode solver.

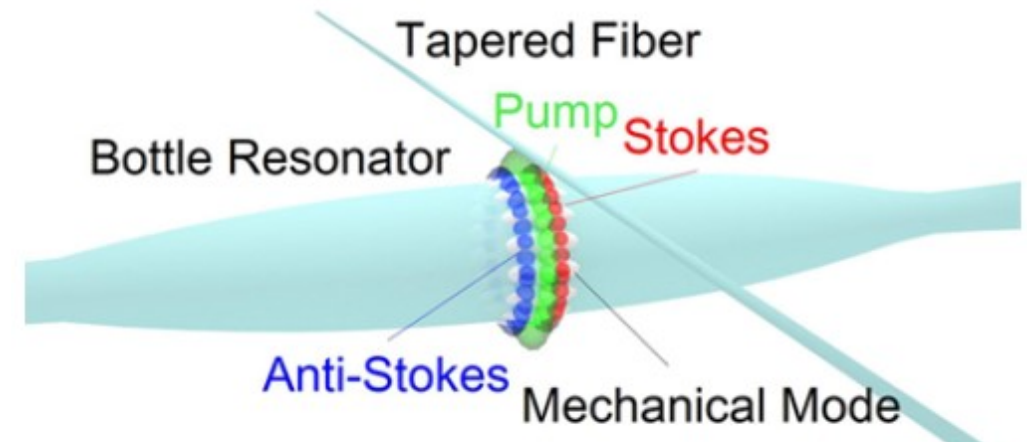

Figure 3.12 An interaction of optical and mechanical modes via radiative pressure of photons. The coupling between the optical pump mode and mechanical mode creates the Stokes and Anti-Stokes fields. The figure has been reproduced with permission of [10].

\subsubsection{Magnetic Field Sensors}

The MBR is a perfect candidate for precise optical, mechanical, physical, chemical, biological, and magnetic field sensors [53-55]. A magnetic field sensor can be created using WGM of the optofluidic MBR (filled with magnetic fluid); see Figure 3.13(a) [56]. COMSOL software is used for simulations, and the field distribution in the $x y$ and $y z$ planes are shown in Figures 3.13(b-c). Once the magnetic fluid is loaded into the 
optofluidic MBR, the magnetic nanoparticles randomly and uniformly disperse inside when no magnetic field is applied, which can be considered a liquid-phase state, as shown in Figure 3.13(d). When a magnetic field is implemented, the nanoparticles form uniform magnetic columns according to magnetic field lines' direction. The magnetic fluid state is changed to the solid-phase, as shown in Figure 3.13(e). Two-phase separation changes the dielectric constant, which changes the refractive index of the magnetic fluid —an increase in the refractive index due to the magnetic fluid results in resonance wavelength to shift.

FFB numerical techniques will be used to compute the response of the MBR as a sensor. The details, particularly as a refractive index, temperature, gas, or chemical sensor, are presented in Chapter 7.

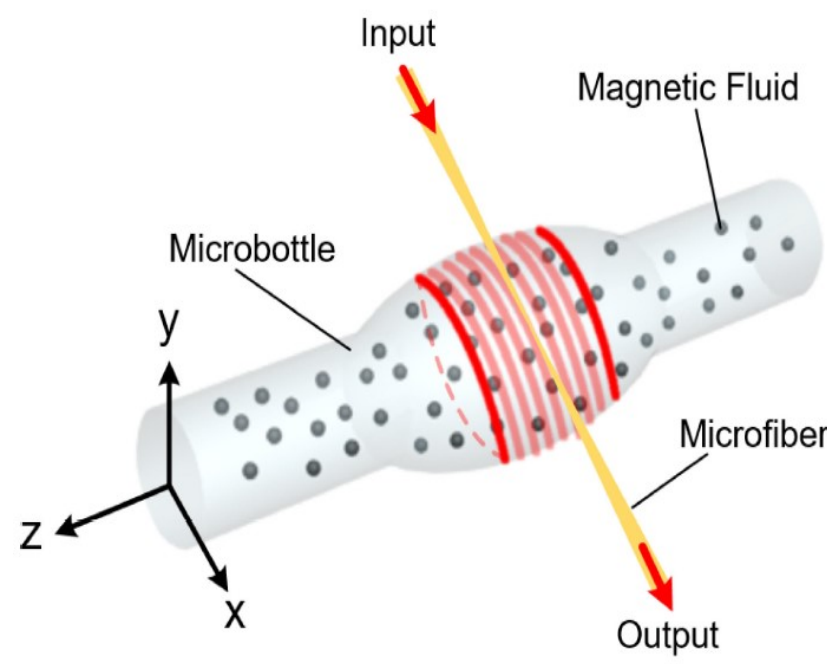

(a)

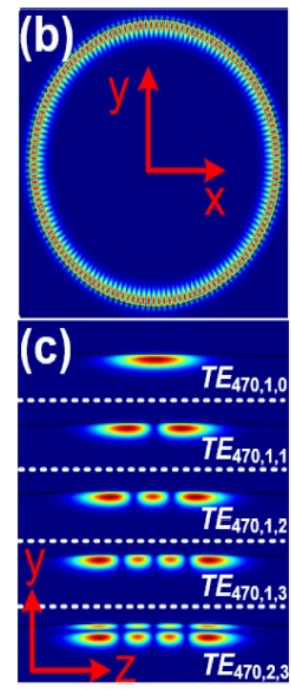

$T E_{470,2}$

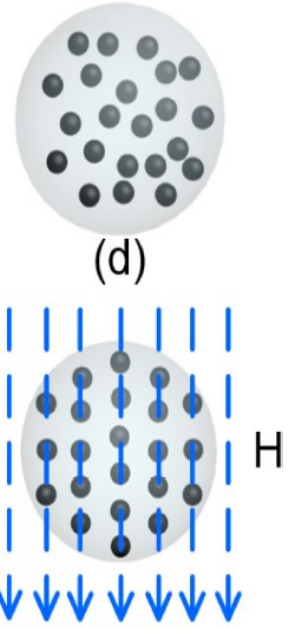

(e)

Figure 3.13 (a) Opto-fluidic MBR filled with magnetic fluid coupled to a tapered fiber.

COMSOL simulations of field distribution of modes in the $x y(b)$ and $y z$ (c) planes. Schematic

diagram of magnetic fluid particles without (d) and with applied magnetic field (e). The figure has been reproduced with permission of [56]. 


\subsection{Hollow Micro-optic Bottle Resonator (HMBR)}

The HMBR is a hollowed-out version of the MBR and is briefly discussed here before closing this chapter. The HMBR is fabricated on a silica capillary. The main steps of the fabrication process are HF etching for wall thinning. Heat and pressure are applied simultaneously during the etching process to form the desired parabolic shape in the axial direction. This fabricated HMBR can be a hybrid of the bottle resonator (considering its profile) and ring resonator (because it is hollow). A schematic is shown in Figure 3.14. The HMBR will have intracavity and WGM features. The intracavity allows internal sensing and can be incorporated as a microfluidic sensor [57], as shown in Figure 3.15. The hollowcore bottle structure can provide an ultra-high $Q$ factor, axial mode alignment, and mode selectivity [54].

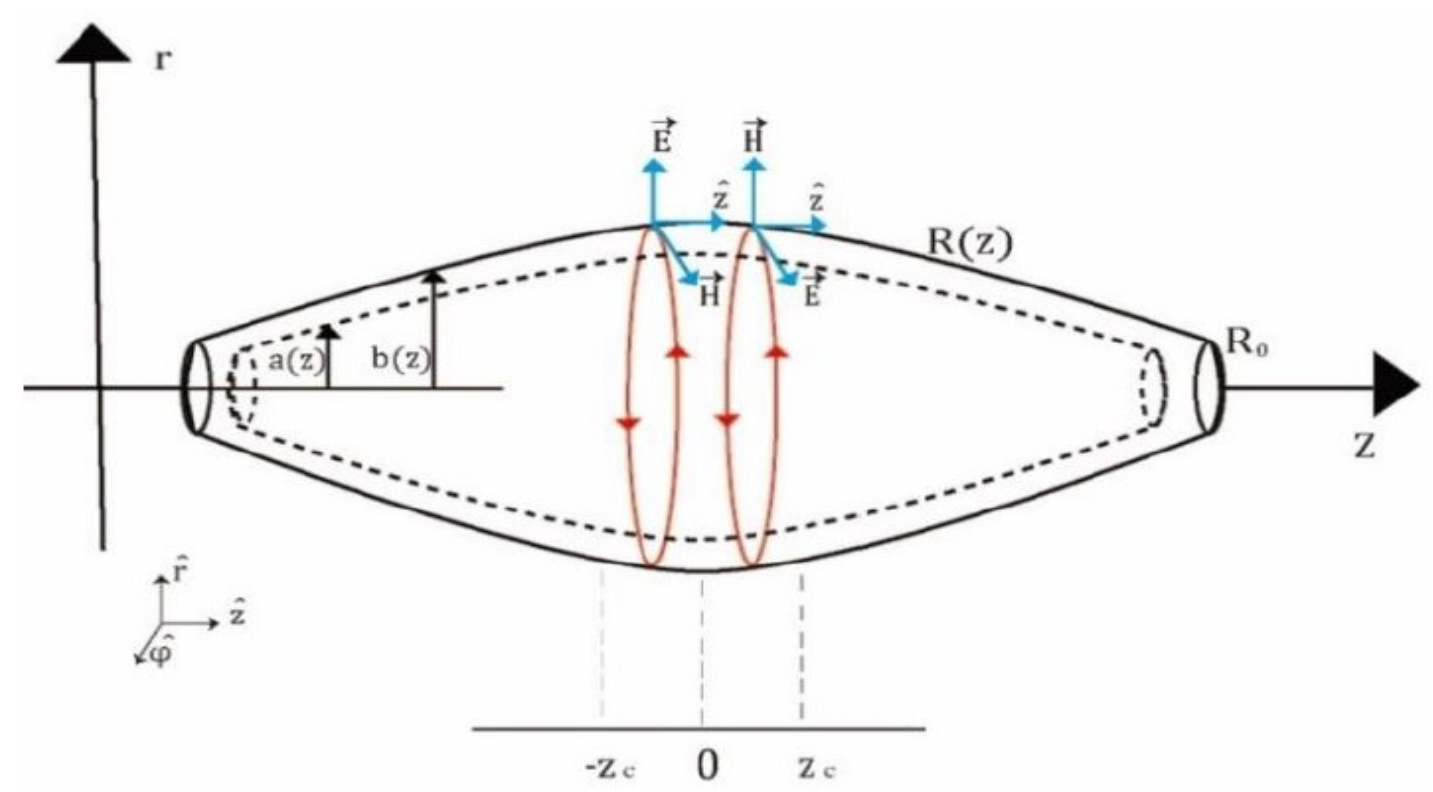

Figure 3.14 A general schematic of a hollow micro-optic bottle resonator. The dotted line represents the inner radius, creating a boundary between the thin walls and hollowed region [61]. 


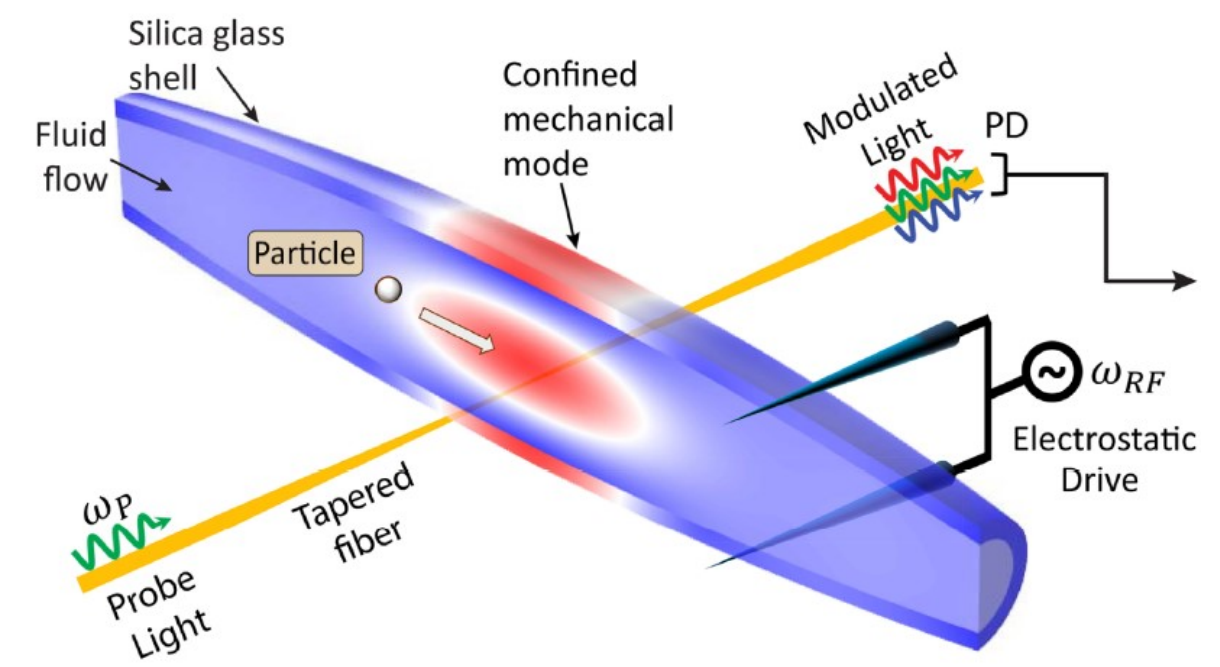

Figure 3.15 The principle of optomechanical fluid detection of particle flowing

through hollow micro capillary resonator. This image has been reproduced with permission from [57]

The interaction of the analyte with the evanescent fields of the WGM results in a change in the resonance mode wavelength (or frequency). The evanescent field occurs when light undergoes TIR at the boundary of two media. The light extends into a lower refractive index medium with an exponential decay in its field at the interface of two media. The WGM of the resonator is excited through a tapered-fiber coupler. When the coupler is closer to the resonator, the incident light will interact with the decaying fields, and the phase-matched condition is satisfied; therefore, the light can now travel from the coupler to the resonator for WGM excitation. The resonator is hollowed on the inside with thin walls permitting the mode's evanescent fields to interact with the inner region's and outer region's analyte at the same time, see Figure 3.16. 

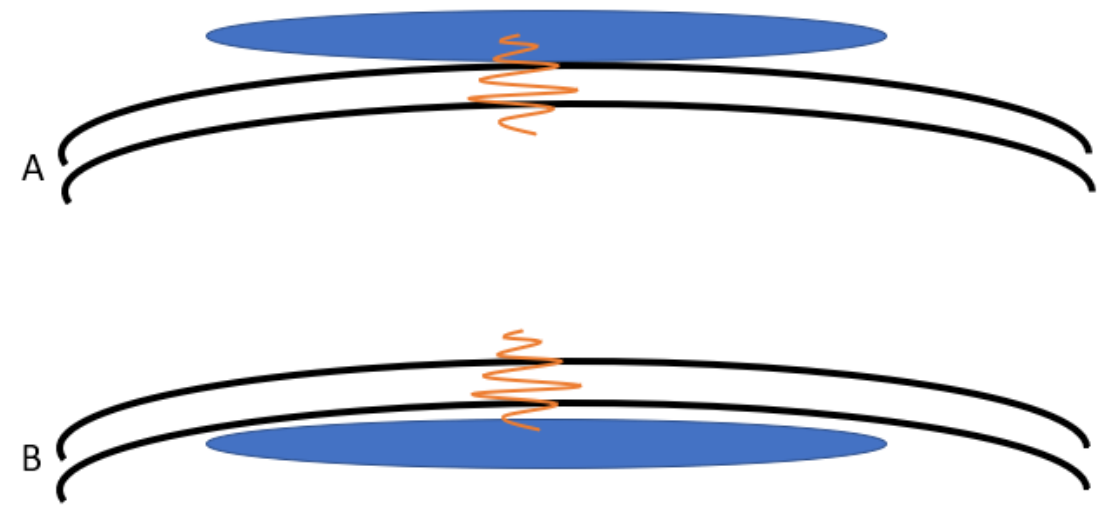

Figure 3.16 The mode is confined in the thin-walls, its evanescent fields interact with the external analyte (A). The HMBR evanescent fields also leak into the inner-core where it interacts with the analyte (B).

This chapter focuses on the MBR structure. The commonly used fabrication techniques of the MBR are presented. A fabrication attempt by the author is also briefly discussed. This effort will be continued by the supervisor (Dr. Gauthier) after the pandemic is over. Professor Levesque at RMC has assembled the experimental system to build MBRs by laser heating. All that is needed is access to the lab. The WGM excitation would require the resonator to couple with the external waveguide, so various coupling techniques are also discussed. At the end of the chapter, the HMBR is briefly discussed. A detailed review is presented on the applications of MBR. Among these applications, the sensor has shown great potential. The author has narrowed down the research field, focusing only on the temperature sensor, gas/refractive index sensor, and microfluidic/chemical sensor. A detailed literature review on these sensors is presented in the upcoming chapter. 


\section{Chapter 4: Literature Review on Optical Sensors}

\subsection{MBR as Sensors}

In recent decades, MBR has been used for precise optical, mechanical, physical, chemical, gas, and biological sensing [58-62]. In contrast to other WGM resonators, the solid MBR can be used as a sensing device where the medium under investigation is situated on the outer surface. In the hollow MBR, the medium under investigation may be either located outside or inside. The optical sensing principle is usually based on the resonance wavelength shift caused by the investigated medium's presence.

Sensitivity and detection limit are typically used to characterize the performance of the sensor. Higher sensitivity with a lower detection limit makes a better sensor. Typically, a slight change in the sensing parameter can cause a shift in the resonance (wavelength), and the sensor sensitivity $(S)$ can be estimated as [63]:

$$
S=\frac{\Delta[\text { Output }]}{\Delta[\text { Input }]}
$$

For example, if $\Delta[$ Input $]$ is the analyte's refractive index change, and $\Delta[$ Output $]$ is the

corresponding wavelength shift $(\Delta \lambda)$, the sensitivity is determined by $S=\frac{\Delta \lambda}{\Delta n}$ in the unit of $\mathrm{nm} / \mathrm{RIU}$ (Refractive Index Unit). The detection limit is the lowest quantity of the analyte that a sensor can detect. It is limited by the spectrum analyzer's sensitivity measurements when monitoring wavelength changes of the resonance and the estimated noise when measuring the least amount of analyte's concentration. The detection limit $(D L)$ can be expressed as: 


$$
D L=k \frac{\sigma}{S}
$$

where $S$ is the sensitivity, $\sigma$ is the concentrated analyte's standard deviation, and $k$ is the uncertainty constant or coverage factor. See Figure 4.1 for the relationship between standard deviation and uncertainty constant. The value for $k$ is associated with the uncertainty constant multiplied by the standard deviation. If $k$ is selected to be 3 , the $D L$ can be considered the lowest analyte amount detected under the experimental conditions as approximately $99.7 \%$ of the analyte lies within three standard deviations of the mean. If $k=2$, there is a $95 \%$ chance of the analyte to be found. If $k=1$ is selected, the detection limit is the same as noise. There is noise because approximately $68 \%$ of the analyte will lie within one standard deviation of the mean. The sensitivity must be increased for a low detection limit, and the noise must be simultaneously decreased [64-65].

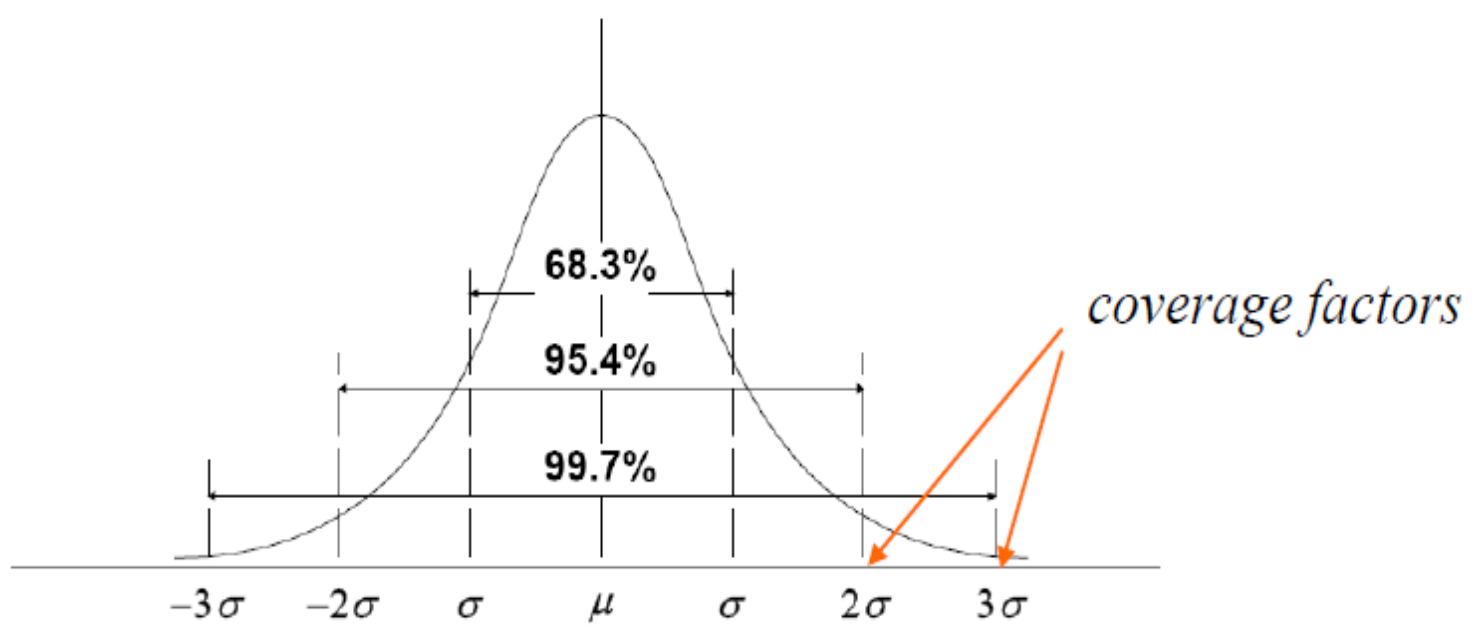

Figure 4.1 The coverage factor, $k=3$, gives $99.7 \%$ certainty that the minimum analyte is present. 


\subsection{Sensor Applications}

A literature review of MBR as a sensor to measure temperature, gas, and chemical constituents is presented in this section. The sensors designed in this thesis are compared to specific types reviewed here.

\subsubsection{Temperature Sensor}

Thermal expansion (or contraction) and thermo-optic effects are two material properties that may change the resonator's size and refractive index when the heat is applied [66].

The coefficient of linear thermal expansion is a material property that is indicative of the extent to which a material expands or contracts as the temperature changes. Usually, an increase in temperature gives rise to the thermal expansion property of the material. However, not all materials expand with increasing temperature, and this property is known as negative thermal expansion. Note, the coefficient of thermal expansion can be positive, negative, or zero. The property of linear thermal expansion, $\alpha$, can be expressed as:

$$
\alpha=\frac{\Delta L}{\mathrm{~L}_{0} \Delta T}
$$

where $\Delta L$ is the change in MBR's length, $L_{0}$ is the initial length, and $\Delta T$ is the temperature change.

In the thermo-optic effect, when the heat is applied to the resonator, the material's refractive index is varied. It is expressed as: 


$$
\beta=\frac{\Delta n}{\mathrm{n}_{0} \Delta T}
$$

where $\Delta n$ is the change in refractive index and $n_{0}$ is the initial refractive index.

In the temperature sensor, the MBR's WGM resonance wavelength can be a function of the refractive index and fiber radius. As the wavelength shifts with the temperature change, the refractive index and the radius are affected. This shift in resonant wavelength can be calculated as:

$$
\Delta \lambda=\alpha+\beta
$$

Below are a few examples of MBR temperature sensors utilizing equation (4.5) to calculate the sensitivity as $S=\frac{\Delta \lambda}{\Delta T}\left(\operatorname{in} \frac{n m}{K}\right)$.

In 2016, the silica MBR was designed with a length of $500 \mu \mathrm{m}$. Simulations were tested for three different temperatures, i.e., $200 \mathrm{~K}, 300 \mathrm{~K}$, and $400 \mathrm{~K}$ as a function of fiber radius. As the radius decreased from $100 \mu \mathrm{m}$ to $10 \mu \mathrm{m}$, the temperature sensitivity decreased $\sim 10 \%$. The MBR temperature sensor has a sensitivity of $10 \mathrm{pm} / \mathrm{K}$ with a temperature detection limit of $1 K[60]$.

In 2016, the MBR was used as a temperature sensor in the laser cooling of solids. Laser cooling of solids investigates the refrigeration of rare earth-doped dielectrics or semiconductors with laser light. Rare earth-doped low phonon energy fibers can be lasercooled. $\mathrm{Yb}^{3+}$-doped ZBLAN fiber and $\mathrm{Yb}^{3+}$-doped YAG fiber were used for the fabrication of MBR. It was placed on the laser-cooled fiber surface to measure the temperature 
distribution along with the laser-cooled sample. The WGM of MBR can be excited with evanescent fields of tapered-fiber. The excited WGM appears in the transmission spectrum due to temperature variations, using $\mathrm{Yb}^{3+}: \mathrm{ZBLAN}$ and $\mathrm{Yb}^{3+}$ : YAG is $\sim 12 \mathrm{pm} / \mathrm{K}$ and $\sim 16 \mathrm{pm} / \mathrm{K}$, respectively [53].

In 2018, MBR placed in plastic packaging was designed with a length of $300 \mu \mathrm{m}$ and axial width of $207 \mu \mathrm{m}$. This packed resonator was tested as a temperature sensor in the range of $290.3 \mathrm{~K}$ to $296.7 \mathrm{~K}$ at $1550 \mathrm{~nm}$. The sensitivity was recorded to be $10.5 \mathrm{pm} / \mathrm{K}$ with a detection limit of $0.06 \mathrm{~K}$ [67].

In 2019, MBR made of silica fiber (hundreds of microns in size) was tested as a temperature sensor. The temperature ranged from $40{ }^{\circ} \mathrm{C}$ to $100{ }^{\circ} \mathrm{C}$. The sensitivity value from the wavelength shift was recorded to be $1.3 \mathrm{pm} /{ }^{\circ} \mathrm{C}$. The experiment was repeated three in the same day to investigate repeatability. The transmission at different temperature levels was recorded for 60 seconds to examine the sensor's stability and accuracy. This experiment's repeatability, stability, and sensitivity show that the MBR may be the right candidate for temperature sensors [68].

In 2020, theoretical work was presented based on CdSe quantum dots-doped PMMA MBR. During the simulations, the MBR's length was kept at $30 \mu m$ and the axial width at $5 \mu \mathrm{m}$. The temperature effects were studied from $-50{ }^{\circ} \mathrm{C}$ to $75^{\circ} \mathrm{C}$. The sensitivity was found to be $9.4 \mathrm{pm} /{ }^{\circ} \mathrm{C}[69]$. 


\subsubsection{Gas/Refractive Index/Pressure Sensor}

Any slight change due to gas compound(s) or bulk change in refractive index in the MBR outer boundary's proximity can shift the WGM's resonance frequency. The shift in frequency (or wavelength) with refractive index change can be used in sensitivity

calculations, i.e., $S=\frac{\Delta \lambda}{\Delta n}$ (in $\frac{n m}{R I U}$ ). Likewise, at constant volume and temperature, the pressure-dependent refractive index changes can be utilized to measure pressure-related sensitivity $S=\frac{\Delta \lambda}{\Delta P}$ (in $\frac{n m}{b a r}$ ). A few of the highest performing gas, refractive index, and pressure sensors are discussed below.

In 2015, the HMBR was fabricated with a wall thickness of $5 \mu \mathrm{m}$, and its internal sensing environment was utilized for detection. In this experiment, the presence of $\mathrm{CH}_{4}$ in the air at atmospheric pressure was detected. It was found that the threshold was less than $0.1 \%[54]$.

In 2016, the MBR (axial width ranges from 10 to $100 \mu \mathrm{m}$ ) was used as a refractive index sensor. During the experiment, it was found that as the radius of the fiber decreased, the sensitivity increased. The sensor showed a refractive index sensitivity of approximately $150 \frac{n m}{R I U}$ for the TM mode and $130 \frac{n m}{R I U}$ for the TE mode. The detection limit for the refractive index was $\sim 6.67 \times 10^{-5}[60]$.

In 2016, the HMBR was designed such that its one end was sealed, and the wall thickness was reduced to around $500 \mathrm{~nm}$. By applying aerostatic pressure at $780 \mathrm{~nm}$ wavelength, the WGM's frequency shifted due to stress-induced refractive index changes 
and strain-induced size expansion. A pressure-frequency sensitivity of $38 \frac{\mathrm{GHz}}{\mathrm{bar}}$ was achieved [59].

In 2019, an orthogonal demodulation sensing system was created using MBR. In this technique, a highly sensitive wavelength was used to lock the laser's wavelength to the MBR's resonant mode. The pressure-frequency sensitivity was found to be $11.6 \frac{\mathrm{GHz}}{\mathrm{bar}}$ with a detection limit of $0.0515 \mathrm{mbar}$, operating at $850 \mathrm{~nm}$ wavelength [70].

\subsubsection{Chemical Sensor}

Usually, an HMBR with a thin wall is preferred when designing a chemical sensor. The walls are thinned down, allowing the evanescent fields to extend significantly into the hollowed core, impacting the potential sensitivity limits. As the chemical flow through the refractive index was varied, so was the resonance wavelength. The chemical sensor's sensitivity was estimated to be $S=\frac{\Delta \lambda}{\Delta n}$ (in $\left.\frac{n m}{R I U}\right)[71]$.

In 2011, the MBR's WGM was used for microfluidic detection, such as water and aqueous ethanol. The change in refractive index records the shift in resonance and gives a sensitivity of $0.5 \frac{\mathrm{nm}}{R I U}[72]$.

In 2016, the core of the HMBR was filled with a mixture of dimethylsulfoxide and water. This mixture's refractive index changed from 1.319 to 1.320 with a step of $2 \times 10^{-4}$. The bulk refractive index sensor showed $18.8 \frac{\mathrm{nm}}{R I U}$ sensitivity and a DL of $5.4 \times 10^{-5}[73]$. 
In 2019, an MBR was designed to have an axial width of $190 \mu m$, where mode excitation of tapered fibers with two different diameters (8 $\mu m$ and $10 \mu m)$ were used. The analyte to be detected in this experiment is an aqueous solution of formaldehyde $\left(\mathrm{CH}_{2} \mathrm{O}\right)$. It was found that using eight-micron bare tapered-fiber could achieve a sensitivity of $3.625 \mathrm{dBm} /$ concentration, and a ten-micron tapered-fiber only achieved $0.278 \mathrm{dBm} /$ concentration [74].

In 2019, a silica HMBR with a wall thickness of 5 to $10 \mu \mathrm{m}$ was fabricated. The internal region was coated with a polymer, and buffer solutions of known $\mathrm{pH}$ were run in and out of the HMBR. Due to changes in varying buffer solutions, the refractive index of the polymer was varied. The shift in polymer wavelength gave a sensitivity of $33 \frac{n m}{R I U}[75]$.

This chapter discussed two essential characteristics of a sensor, i.e., sensitivity and detection limit, following by a literature review on MBRs as temperature, gas, refractive index, pressure, and chemical constituent sensors. This encourages further research for this thesis's findings. The author will feature the solid MBR and hollow MBR in upcoming chapters, which show improved sensitivity due to their unique parameters compared with those presented here.

The next chapter discusses numerical techniques such as FEM, FDTD, PWEM, and FFB. Later in the next chapter, theoretical development based on FFB is explained. This leads to the eigenvalue equation that provides the complex modal frequencies and field profiles for the proposed MBR. 


\section{Chapter 5: Numerical Techniques}

There are several techniques available for numerical computations. The most commonly used are the Finite-Element-Method (FEM) [76], Finite-Difference TimeDomain FDTD [77], and spectral analysis such as the Plane Wave Expansion Method PWEM [78]. Each of them has distinct advantages and disadvantages. FDTD is known for flexibility in the time domain, PWEM for efficiency with a periodic structure in the frequency domain, and FEM for accuracy. The theory and application of the techniques mentioned above are reviewed below. The computational technique utilized in this thesis is known as the Fourier-Fourier-Bessel (FFB) mode solver. It closely resembles PWEM's formulation approach on a cylindrical coordinate system [79].

\subsection{Finite-Element-Method}

FEM is a powerful and versatile technique for handling complex materials and inhomogeneous problems implemented in the frequency domain. It requires volume discretization, which is the most critical step, because it will affect the computer storage requirements, computational time, and numerical results accuracy.

The entire computational space is divided into a fine mesh of non-overlapping small subdomains, usually referred to as elements. In the one-dimensional problem, the elements are short line segments interconnected to form the original line, as shown in Figure 5.1(a). The elements are usually small triangles or rectangles for two-dimensional simulations, as shown in Figure 5.1(b). In a three-dimensional problem, space may be subdivided into tetrahedra, triangular prisms, or rectangular bricks, as shown in Figure 5.1(c). 


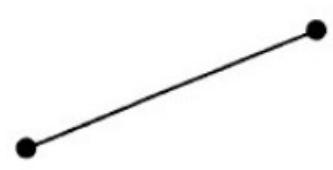

(a)

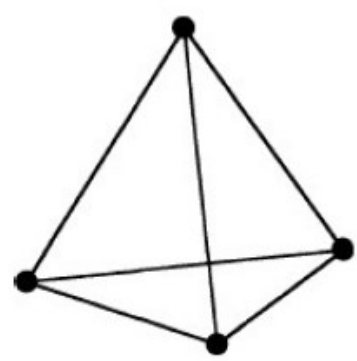

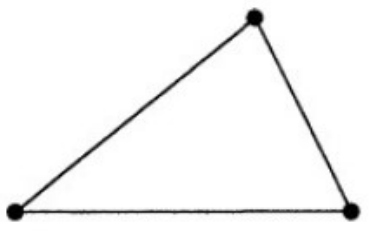

(b)

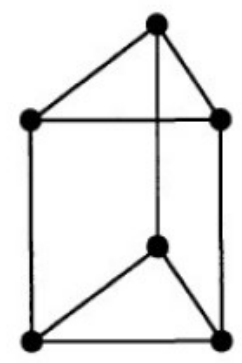

(c)
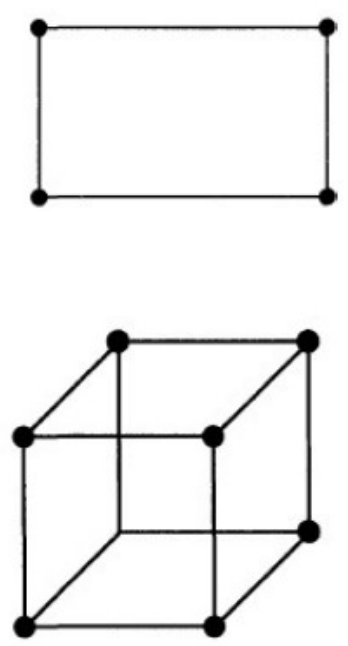

Figure 5.1 Basic finite elements are shown in (a) one-dimension, (b) two-dimensions, and (c) three-dimensions.

In finite element solutions, the problem is formulated in terms of an unknown function $\Phi$ at nodes (number of vertices) associated with the elements. For example, a line element has two nodes, one at each endpoint. A triangle has three nodes, whereas a tetrahedron has four nodes located at their corners. The second step is to select the interpolation function, which approximates the unknown solution within an element. The interpolation is usually chosen to be a polynomial of a first, second, or higher order. The third step is to formulate the system of equations and apply the required boundary conditions. Obtaining the final solution is the most time-consuming step in finite element analysis. The equations form a system as:

$$
[K][\Phi]=[b]
$$


where $[K]$ is a global matrix composed of equations with several equal or multiple order nodes from mesh to finite elements, $[\Phi]$ is a vector of unknown values (of electric and magnetic fields for EM analysis), and $[b]$ is the source vector which depends on field sources' intensities and imposed boundary conditions.

This thesis presents 3-D resonating structures, and if the simulations are based on the implementation of FEM, it would require the solution of a global sparse linear system of equations over the entire computational space. The approximate solution is applied for each element to build a matrix and then solve the appropriate boundary conditions. It would require very large computer memory resources when solving the equation set over the resonator structures. This makes FEM ill-suited for obtaining accurate and rapid results for the structural examination of the MBR.

\subsection{Finite-Difference Time-Domain}

Dr. Yee originally proposed the FDTD method in 1966 as a discrete solution to Maxwell's equations based on central difference approximations of the curl-equations' spatial and temporal derivatives [80]. His proposed approach's novelty was the staggering of the electric and magnetic fields in both space and time to obtain second-order accuracy. In 1975, Taflove and others extended Dr. Yee's method to simulate dielectric cylinders' scattering [81-82].

The FDTD technique is a robust method commonly used when flexibility is required in numerical simulations. The finite difference method of solving differential 
equations discretizes the function and derivatives such that a finite difference approximates them. The derivative of $f(x, y)$ for $x$ is written here as a central difference expression:

$$
\frac{\partial f(x, y)}{\partial x}=\frac{f\left(x+\frac{\Delta x}{2}, y\right)-f\left(x-\frac{\Delta x}{2}, y\right)}{\Delta x}
$$

where the $\Delta x$ is the size of the spatial discretization.

When working with Maxwell's equations, the discretized quantities are the fields $(\vec{E}, \vec{H})$, material properties, and time. Maxwell's equations with no external sources for dielectric media (non-magnetic) are given by:

$$
\begin{aligned}
& \vec{\nabla} \times \vec{H}(r, t)=\epsilon_{r}(r) \epsilon_{o} \frac{\partial \vec{E}(r, t)}{\partial t} \\
& \vec{\nabla} \times \vec{E}(\mathrm{r}, \mathrm{t})=-\mu_{\mathrm{o}} \frac{\partial \overrightarrow{\mathrm{H}}(\mathrm{r}, \mathrm{t})}{\partial \mathrm{t}}
\end{aligned}
$$

where $\vec{H}$ and $\vec{E}$ are the magnetic and electric fields, $\epsilon_{r}(r)$ is the relative permittivity, and $\epsilon_{o}$ and $\mu_{o}$ are the permittivity and permeability of free space, respectively. Referring to Dr. Yee's method, the fields are discretized on overlapping grids known as the Yee cell. An example is shown in Figure 5.2 for TM polarization with components $\left(H_{x}, H_{y}, E_{z}\right)$. A similar profile could be produced for TE polarization with components $\left(E_{x}, E_{y}, H_{z}\right)$. The $\vec{E}$ and $\vec{H}$ fields are interleaved around a cell whose origin is at locations $i, j$ and $k$. Every $\vec{E}$ field is located half a cell width from the origin in the direction of its orientation, denoted by a circle; every $\vec{H}$ field is offset half a cell in each direction except that of its orientation, denoted by an arrow. 


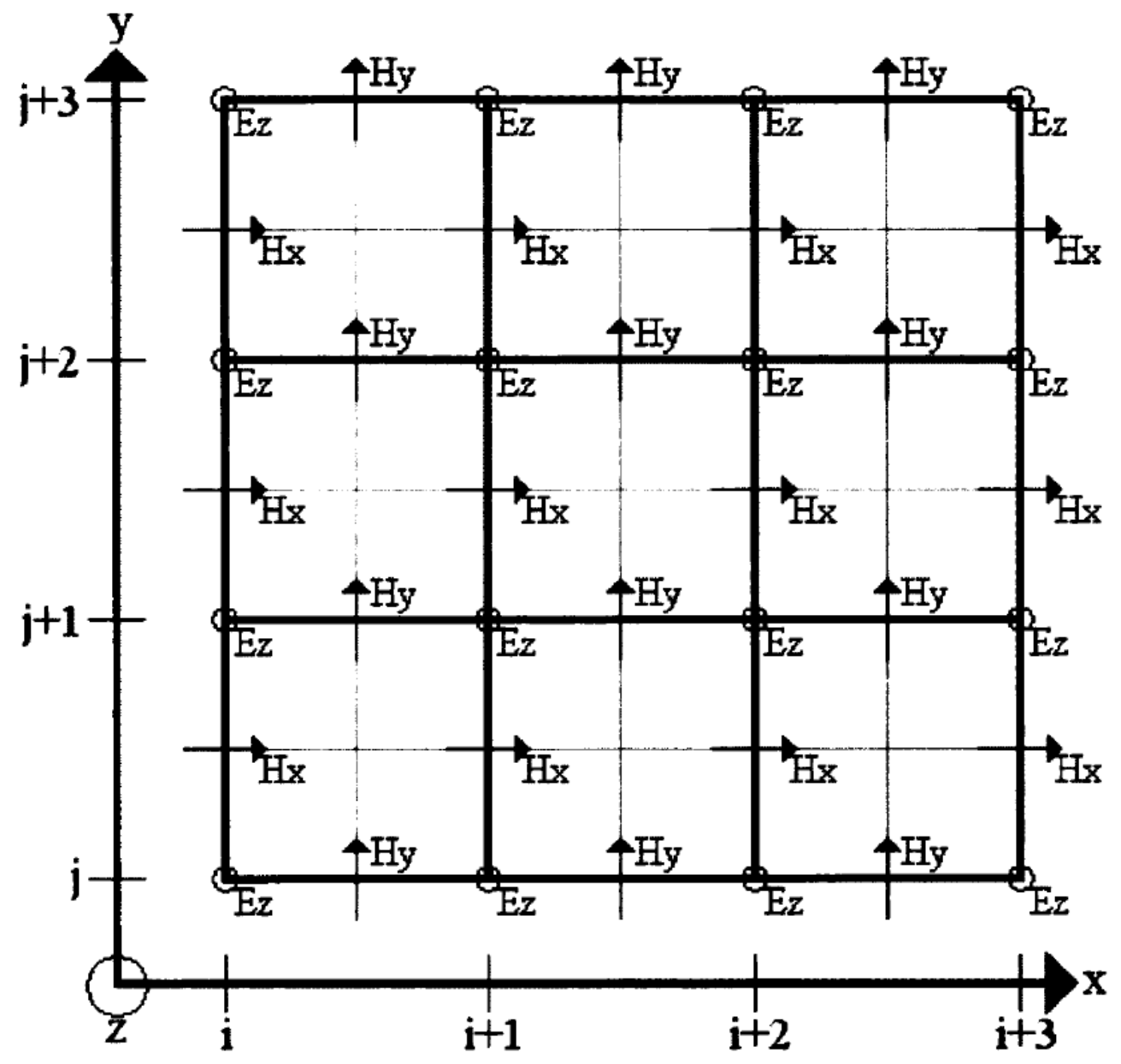

Figure 5.2 The Yee cell for TM polarization. The $\vec{E}$ and $\vec{H}$ fields are discretized on overlapping grids and used in finite difference expressions of Maxwell's curl equations [78].

\subsubsection{Accuracy and Stability}

Stability results from energy propagation within a computational cell causing fields to diverge. The accumulation occurs as energy flows across the spatial cell, defined by spatial discretization, and into adjoining cells with each $\Delta t$ time step. If the time step is too large, the energy cannot propagate across the cell and will accumulate. The stability 
condition places an upper limit on the spatial grid spacing and is determined by the speed of light, time, and spatial discretization. It is known as the Courant Factor, and in 3-D, it is given as:

$$
c \Delta t \leq \frac{1}{\sqrt{\frac{1}{(\Delta x)^{2}}+\frac{1}{(\Delta y)^{2}}+\frac{1}{(\Delta z)^{2}}}}
$$

where $\Delta x, \Delta y$, and $\Delta z$ are the largest spatial discretization in the $x, y$ and $z$ directions permitted in a stable simulation. Mode and time evolution of the $\vec{E}$ and $\vec{H}$ fields through the grid are determined by leapfrogging (skip one point and move to the other) their calculations with each time step. The time information at the grid points is computed and can be logged as a function of the time step integer and time increment $\Delta t$. This spectral information can be obtained through a temporal Fourier transform.

The accuracy is related to the resolution of the spatial grid. If the discretization grid is not sufficiently fine for the feature sizes being modelled, the simulated structure will not match the actual design under test.

\subsubsection{Absorbing Boundary Condition}

A difficulty arises when the FDTD method is applied to open or unbounded computational domains. The simulation region is truncated such that it encloses the scatterer and is extended on its border by an absorbing layer (ABC). A Perfectly Matched Layer (PML) is a type of $\mathrm{ABC}$ truncation technique. The objective is to attenuate the propagated fields within the PML without a reflected component at the free space PML interface. Thus, FDTD and PML are said to be perfectly matched [83-84]. 
FDTD has advantages of robustness, adaptability to complex geometries, ease of understanding and implementation, and flexibility when solving Maxwell's equation in the time domain. One of the disadvantages of the FDTD method is that it requires a full discretization of the electric and magnetic fields throughout the entire computational domain volume. If the volume space has a significant amount of free space, FDTD is forced to compute over it. Another challenge FDTD faces is when a system has a narrowband slow decaying resonance with a very high $Q$. Simulations in such conditions result in a very long computational time. For the reasons mentioned above, FDTD may not be the best technique for this thesis as we are searching for long-lived resonant states.

\subsection{Plane-Wave Expansion Method}

The PWEM is a basis expansion technique through which both the fields $(\vec{E}, \vec{H})$ and material properties are expressed as a series of plane waves. This method relies on dielectric periodicity, which imposes a periodicity on the supported modes. The theory addressing the behavior of waves in periodic media is known as Bloch theory [85]. When PWEM is incorporated with Maxwell's equations, the series representation allows the equation to be rearranged as an eigenvalue expression where the Expansion coefficient of fields are the eigenvectors, and the frequencies are obtained from the eigenvalues.

A 3-D lattice has lattice vectors $\left(\overrightarrow{a_{1}}, \overrightarrow{a_{2}}, \overrightarrow{a_{3}}\right)$ and a reciprocal lattice in k-space such that $\overrightarrow{a_{\imath}} \cdot \overrightarrow{b_{J}}=2 \pi \delta_{i j}$, where $\delta_{\mathrm{ij}}$ is the Kronecker delta. An expansion of the reciprocal lattice vector is $\vec{G}_{\mathrm{m}}=\mathrm{m}_{1} \vec{b}_{1}+\mathrm{m}_{2} \vec{b}_{2}+\mathrm{m}_{3} \vec{b}_{3}$, where $\left(\mathrm{m}_{1}, \mathrm{~m}_{2}, \mathrm{~m}_{3}\right)$ are integers. The expansions for any component of $\vec{E}$ or $\vec{H}$ fields and the inverse of relative permittivity are expressed as: 


$$
\begin{aligned}
& (\vec{E}, \vec{H})=\sum_{G_{m}}\left(\kappa_{G_{m}}^{E}, \kappa_{G_{m}}^{H}\right) e^{j \vec{G}_{m} \cdot \vec{r}} e^{j \vec{k} \cdot \vec{r}} \\
& \frac{1}{\varepsilon_{r}(\vec{r})}=\sum_{G_{n}} \kappa_{G_{n}}^{\varepsilon} e^{j \vec{G}_{n} \cdot \vec{r}}
\end{aligned}
$$

whereon reciprocal lattice vectors $\mathrm{G}_{\mathrm{m}}$ and $\mathrm{G}_{\mathrm{n}}$ sums are calculated, $\kappa_{m}^{\text {Field }}$ and $\kappa_{n}^{\varepsilon}$ are the expansion coefficients of the field $(\vec{E}, \vec{H})$ components and material properties, respectively. The expansions are performed over the smallest unit of periodicity in the reciprocal lattice, known as the Brillouin zone [86]. Using Bloch's theory and the Fourier expansion, it can be mathematically assumed that the Brillouin zones are infinitely repeated. When resonator modes are modelled, it is ensured that the supercell around the cavity is such that the mode's fields are zero at the boundary, provided no coupling between the repeated cavities. When reconstructing a modal field profile, the eigenvector that corresponds to the eigenvalue includes the expansion coefficients for the fields, $e_{G_{m}}^{\text {Field }}$, is to be used in (5.6).

The PWEM method is suitable for the analysis of various resonator configurations. However, a structure that does not have translational symmetry requires a supercell approach. The issue with the implementation of supercells is that the number of plane waves required to maintain converged results can consume significant computational resources [78]. PWEM could be utilized for the resonator structures examined in this thesis but need a very large 3-D supercell and long computational time.

To retain the ease of use of the PWEM, an alternate formulation was utilized where the coordinate system and basis functions better match the structure's geometry. This 
relaxes the supercell requirement and reduces the computation times and memory requirements.

\subsection{Fourier-Fourier-Bessel}

The FFB expansion method for modelling resonators used in this thesis is a spectral expansion approach similar to PWEM. The FFB is commonly used in polar and cylindrical coordinates. It was previously used as pattern analysis [87] and pattern recognition [88]. The basis has been useful in describing beam and field profiles in the description of Bessel beams [89]. The most common application is its use in modelling fields within close proximity of circular scatterers [90].

This thesis's theoretical development is based on the FFB numerical mode solver for structures that display a high degree of cylindrical symmetry [91]. This approach is well suited for PCs, optical fibers, ring resonators, and whispering gallery mode-based resonator configurations. When a non-propagating state is desired, a resonator-based device configuration is selected to calculate the field profiles, polarization, frequencies, wavelength, attenuation coefficient, quality factor, and FSR. The usual numerical solver (FDTD and PWEM) can provide such information, but they rely on propagating fields to determine non-propagating standing wave information [92]. In FDTD, the supercell implementation on the computational domain to satisfy resonator state boundary conditions needs high discretization resolution. In PWEM, a vast number of plane waves are required for the computational process. Both techniques could exceed computer resources or require an extremely long simulation time before results are available for examination. Hence, a 
steady state numerical solver using non-propagating fields has been developed from Maxwell's equations cast in the frequency domain and using a set of Fourier-Bessel functions as the basis space for structures that conform to cylindrical symmetry.

The exploitation of the symmetries and the ability to solve for specific mode families make this technique extremely efficient (memory and time) in determining the resonator structure's steady states. The details on the FFB can also be found in several application-oriented publications [93-95]. In effect, FFB and PWEM generate a system of equations that can be cast into an eigenvalue formulation. This numerical solver in cylindrical coordinates utilizes a Fourier-Bessel basis function series expansion for the material and optical fields. It has been dramatically simplified using Faraday's and Ampere's laws rather than usual wave equations. The FFB solver requires the resolution of the electric and magnetic medium properties, and only the first derivatives of the field components give this approach faster convergence properties than using the wave equations directly [96].

\subsubsection{Theoretical Development}

The steps for developing the system matrix and expressions in cylindrical coordinates for the FFB are presented in this section. Bessel functions are used in the radial direction $(r)$ and Fourier basis functions in the angular orientation $(\varphi)$ and out-of-plane

direction $(z)$. A dielectric structure within a cylindrical boundary is shown in Figure 5.3. $R$ is the radial limit in the $(r, \varphi)$ plane. The domain has a height $T$ centered on the cylindrical coordinate system. 


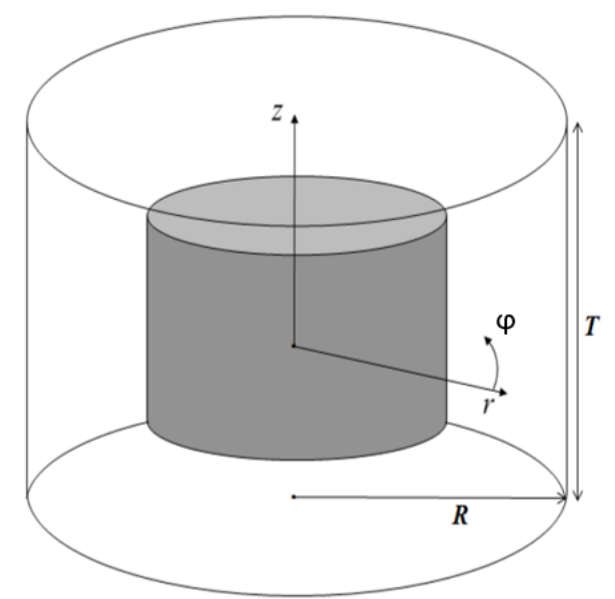

Figure 5.3 Cylindrical computational domain.

The development of the matrix populating expressions when the media demonstrate electric and magnetic anisotropy is presented below for structures that are charge- and current-free and for fields that display a $e^{-j \omega t}$ time dependence where $\omega$ is the frequency and $c$ is the speed of light in a vacuum. The notation is simplified and symmetrized by setting $\overrightarrow{\mathfrak{I}}=j \sqrt{\frac{\mu_{o}}{\varepsilon_{o}}} \vec{H}$

$$
\overleftrightarrow{\mu_{r}^{-1}} \nabla \times \vec{E}=\frac{\omega}{c} \overrightarrow{\mathfrak{I}} \quad \overleftrightarrow{\varepsilon_{r}^{-1}} \nabla \times \overrightarrow{\mathfrak{I}}=\frac{\omega}{c} \vec{E}
$$

These expressions utilize the inverse of the relative permittivity and permeability tensors when anisotropy is included and, in general, contain nine elements that must be specified at every point in the computational domain [97].

$$
\overleftrightarrow{\mu_{r}-1}=\left[\begin{array}{lll}
\mu_{11}^{-1} & \mu_{12}^{-1} & \mu_{13}^{-1} \\
\mu_{21}^{-1} & \mu_{22}^{-1} & \mu_{23}^{-1} \\
\mu_{31}^{-1} & \mu_{32}^{-1} & \mu_{33}^{-1}
\end{array}\right] \quad \overleftrightarrow{\varepsilon_{r}^{-1}}=\left[\begin{array}{lll}
\varepsilon_{11}^{-1} & \varepsilon_{12}^{-1} & \varepsilon_{13}^{-1} \\
\varepsilon_{21}^{-1} & \varepsilon_{22}^{-1} & \varepsilon_{23}^{-1} \\
\varepsilon_{31}^{-1} & \varepsilon_{32}^{-1} & \varepsilon_{33}^{-1}
\end{array}\right]
$$


The inverse of the material properties profiles over the cylindrical space computation domain is series expanded using the FFB expansion series with normalized basis functions $C_{q p n} J_{o}\left(\rho_{p} \frac{r}{R}\right) e^{j q \varphi} e^{j G_{n} z}$, where $C_{q p n}=1 / J_{1}\left(\rho_{p}\right)$ is the normalization constant.

$$
\left[\begin{array}{c}
\varepsilon_{a b}^{-1} \\
\mu_{a b}^{-1}
\end{array}\right]=\sum_{q p n}\left[\begin{array}{l}
\kappa_{\varepsilon_{a b}} \\
\kappa_{\mu_{a b}}
\end{array}\right] C_{q p n} J_{o}\left(\rho_{p} \frac{r}{R}\right) e^{j q \varphi} e^{j G_{n} z}
$$

The integer indices, $q p n$, identify various basis functions with $p$ linked to the radial expansion and the Bessel functions, $q$ to the angular Fourier expansion, and $n$ to the axial direction. The $\kappa^{\prime} s$ are the components expansion coefficients. The occurrence of $\rho_{p}$ corresponds to the $p^{\text {th }}$ zero of the lowest-order Bessel function $\left(J_{0}\right)$, and $G_{n}=n \frac{2 \pi}{T}$ is an integer indexed $z$-axis only reciprocal lattice "vector". Note that the series representation of the material tensors utilizes cylindrical coordinate-based basis functions. Thus, the elements of the inverse permittivity tensor profiles and inverse permeability profiles must be converted to cylindrical coordinate representation through suitable coordinate rotation operators. In general, the material tensors are not diagonal in cylindrical space, even though they may be diagonal in an $(X Y Z)$ principle axis coordinate system.

The electric and magnetic field vectors can also be expressed over the same basis function space using the series:

$$
\begin{aligned}
& \vec{E}=\sum_{q p n} \overrightarrow{\kappa_{E}} C_{q p n} J_{o}\left(\rho_{p} \frac{r}{R}\right) e^{j q \varphi} e^{j G_{n} z} e^{j k_{z} z} \\
& \overrightarrow{\mathfrak{I}}=\sum_{q p n} \overrightarrow{\kappa_{\mathfrak{I}}} C_{q p n} J_{o}\left(\rho_{p} \frac{r}{R}\right) e^{j q \varphi} e^{j G_{n} z} e^{j k_{z} z}
\end{aligned}
$$


with the $\vec{\kappa}$ representing the expansion coefficients of the field components collected into three component vectors. Also included in the field series is the possibility of axially directed field propagation with propagation constant $k_{z}$.

The curl of the electric field, $\vec{\nabla} \times \vec{E}$, of (5.8-left) in cylindrical coordinates, produces the following three terms:

$$
\begin{aligned}
& \left(\frac{1}{r} \frac{\partial E_{z}}{\partial \varphi}-\frac{\partial E_{\varphi}}{\partial z}\right)=E_{\nabla \times}(r)=E_{\nabla \times}(r \varphi)+E_{\nabla \times}(r z) \\
& \left(\frac{\partial E_{r}}{\partial z}-\frac{\partial E_{z}}{\partial r}\right)=E_{\nabla \times}(\varphi)=E_{\nabla \times}(\varphi r)+E_{\nabla \times}(\varphi z) \\
& \frac{1}{r}\left(\frac{\partial\left(r E_{\varphi}\right)}{\partial r}-\frac{\partial E_{r}}{\partial \varphi}\right)=E_{\nabla \times}(z)=E_{\nabla \times}(z \varphi)+E_{\nabla \times}(z r)
\end{aligned}
$$

Equations (5.13), (5.14), and (5.15) are combined and collected in matrix form and give:

$$
[\vec{\nabla} \times \vec{E}]=\left[\begin{array}{ccc}
0 & E_{\Downarrow_{\nabla \times}}(r \varphi) & E_{\Downarrow_{\nabla \times}}(r z) \\
E_{\Downarrow_{\nabla \times}}(\varphi r) & 0 & E_{\Downarrow_{\nabla \times}}(\varphi z) \\
E_{\Downarrow_{\nabla \times}}(z r) & E_{\Downarrow_{\nabla \times}}(z \varphi) & 0
\end{array}\right]\left[\begin{array}{c}
\kappa_{E r} \\
\kappa_{E \varphi} \\
\kappa_{E z}
\end{array}\right]
$$

The symbol $\Downarrow$ is used to indicate that the curl operation is cast in operator form with the expansion coefficients of the field extracted and used to build the column vector.

Faraday's law in matrix operator form is: 


$$
\begin{aligned}
& \overleftrightarrow{\mu_{r}-1}[\nabla \times \vec{E}]=\left[\begin{array}{ccc}
\mu_{11}^{-1} & \mu_{12}^{-1} & \mu_{13}^{-1} \\
\mu_{21}^{-1} & \mu_{22}^{-1} & \mu_{23}^{-1} \\
\mu_{31}^{-1} & \mu_{32}^{-1} & \mu_{33}^{-1}
\end{array}\right]\left[\begin{array}{ccc}
0 & E_{\Downarrow \times}(r \varphi) & E_{\Downarrow_{\nabla \times}}(r z) \\
E_{\Downarrow_{\nabla \times}}(\varphi r) & 0 & E_{\Downarrow_{\nabla \times}}(\varphi z) \\
E_{\Downarrow_{\nabla \times}}(z r) & E_{\Downarrow_{\nabla \times}}(z \varphi) & 0
\end{array}\right]\left[\begin{array}{c}
\kappa_{E r} \\
\kappa_{E \varphi} \\
\kappa_{E z}
\end{array}\right]= \\
& \frac{\omega}{c}\left[\begin{array}{l}
\kappa_{\mathfrak{I} r} \\
\kappa_{\mathfrak{\Im} \varphi} \\
\kappa_{\mathfrak{I} z}
\end{array}\right]=\frac{\omega}{c}[\overrightarrow{\mathfrak{I}}]
\end{aligned}
$$

The product of the square matrices on the left-hand side that defines the matrix populating expressions is obtained from:

$$
\begin{aligned}
& {\left[E_{\text {block }}\right]} \\
& =\left[\begin{array}{lll}
{\left[\mu_{12}^{-1} E_{\Downarrow_{\nabla \times}}(\varphi r)+\mu_{13}^{-1} E_{\Downarrow_{V \times}}(z r)\right]} & {\left[\mu_{11}^{-1} E_{\Downarrow_{\nabla \times}}(r \varphi)+\mu_{13}^{-1} E_{\Downarrow \times}(z \varphi)\right]} & {\left[\mu_{11}^{-1} E_{\Downarrow_{\nabla \times}}(r z)+\mu_{12}^{-1} E_{\Downarrow_{\nabla \times}}(\varphi z)\right]} \\
{\left[\mu_{22}^{-1} E_{\Downarrow_{\nabla \times}}(\varphi r)+\mu_{23}^{-1} E_{\Downarrow_{\nabla \times}}(z r)\right]} & {\left[\mu_{21}^{-1} E_{\Downarrow_{\nabla \times}}(r \varphi)+\mu_{23}^{-1} E_{\Downarrow_{\nabla \times}}(z \varphi)\right]} & {\left[\mu_{21}^{-1} E_{\Downarrow_{\nabla \times}}(r z)+\mu_{22}^{-1} E_{\Downarrow_{\nabla \times}}(\varphi z)\right]} \\
{\left[\mu_{32}^{-1} E_{\Downarrow_{\nabla \times}}(\varphi r)+\mu_{33}^{-1} E_{\Downarrow_{\nabla \times}}(z r)\right]} & {\left[\mu_{31}^{-1} E_{\Downarrow_{\nabla \times}}(r \varphi)+\mu_{33}^{-1} E_{\Downarrow_{\nabla \times}}(z \varphi)\right]} & {\left[\mu_{31}^{-1} E_{\Downarrow_{\nabla \times}}(r z)+\mu_{32}^{-1} E_{\Downarrow_{\nabla \times}}(\varphi z)\right]}
\end{array}\right]
\end{aligned}
$$

The matrix element generating expressions are derived from (5.17) using the matrix product of (5.18), multiplication by the complex conjugate of a basis function and the complex conjugate of the axial propagation factor exponential, and integration over the entire computation domain. The matrix generating expressions are collected as:

$$
\begin{aligned}
& {\left[\mu_{i 2}^{-1} E_{\Downarrow_{\nabla \times}}(\varphi r)+\mu_{i 3}^{-1} E_{\Downarrow_{\nabla \times}}(z r)\right]=E_{i 1}(1)+E_{i 1}(2)} \\
& {\left[\mu_{i 1}^{-1} E_{\Downarrow_{\nabla \times}}(r \varphi)+\mu_{i 3}^{-1} E_{\Downarrow_{\nabla \times}}(z \varphi)\right]=E_{i 2}(1)+E_{i 2}(2)} \\
& {\left[\mu_{i 1}^{-1} E_{\Downarrow_{\nabla \times}}(r z)+\mu_{i 2}^{-1} E_{\Downarrow \nabla \times}(\varphi z)\right]=E_{i 3}(1)+E_{i 3}(2)}
\end{aligned}
$$

with the following six expressions: 


$$
\begin{aligned}
& {\left[\mu_{i 2}^{-1} E_{\Downarrow \nabla \times}(\varphi r)\right]\left[\kappa_{E r}\right] \Leftrightarrow\left[\left\{j \sum_{E, \mu} G_{n}^{\prime} \kappa_{\mu_{i 2}}\left\langle\overline{T_{1}}\left(p_{E}, p_{\mu}, p^{*}\right)\right\rangle \delta\left[\begin{array}{c}
q_{E}+q_{\mu}, q^{*} \\
n_{E}+n_{\mu}, n^{*}
\end{array}\right]\right\}\right]\left[\kappa_{E r}\right]} \\
& {\left[\mu_{i 1}^{-1} E_{\Downarrow_{\nabla \times}}(r \varphi)\right] \Leftrightarrow\left\{-j \sum_{E, \mu} G^{\prime}{ }_{n} \kappa_{\mu_{i 1}}\left\langle\overline{T_{1}}\left(p_{E}, p_{\mu}, p^{*}\right)\right\rangle \delta\left[\begin{array}{l}
q_{E}+q_{\mu}, q^{*} \\
n_{E}+n_{\mu}, n^{*}
\end{array}\right]\right\}} \\
& {\left[\mu_{i 1}^{-1} E_{\Downarrow \nabla \times}(r z)\right] \Leftrightarrow\left[\frac{j}{R} \sum_{E, \mu}(q) \kappa_{\mu_{i 1}} T_{0}\left(p_{E}, p_{\mu}, p^{*}\right) \delta\left[\begin{array}{l}
q_{E}+q_{\mu}, q^{*} \\
n_{E}+n_{\mu}, n^{*}
\end{array}\right]\right]} \\
& {\left[\mu_{i 3}^{-1} E_{\Downarrow \nabla \times}(z r)\right] \Leftrightarrow\left[\frac{-j}{R} \sum_{E, \mu}(q) \kappa_{\mu_{i 3}} T_{0}\left(p_{E}, p_{\mu}, p^{*}\right) \delta\left[\begin{array}{c}
q_{E}+q_{\mu}, q^{*} \\
n_{E}+n_{\mu}, n^{*}
\end{array}\right]\right.} \\
& {\left[\mu_{i 3}^{-1} E_{\Downarrow \nabla \times}(z \varphi)\right] \Leftrightarrow\left[\begin{array}{c}
\left\{\frac{1}{R} \sum_{E, \mu} \kappa_{\mu_{i 3}} T_{o}\left(p_{E}, p_{\mu}, p^{*}\right) \delta\left[\begin{array}{c}
q_{E}+q_{\mu}, q^{*} \\
n_{E}+n_{\mu}, n^{*}
\end{array}\right]\right\}+ \\
\left\{\frac{-1}{R} \sum_{E, \mu}\left(\rho_{p}\right) \kappa_{\mu_{i 3}} U_{1}\left(p_{E}, p_{\mu}, p^{*}\right) \delta\left[\begin{array}{l}
q_{E}+q_{\mu}, q^{*} \\
n_{E}+n_{\mu}, n^{*}
\end{array}\right]\right\}
\end{array}\right]} \\
& {\left[\mu_{i 2}^{-1} E_{\Downarrow \nabla \times}(\varphi z)\right] \Leftrightarrow \sum_{E, \mu}\left(\frac{\rho_{p}}{R}\right) \kappa_{\mu_{i 2}} U_{1}\left(p_{E}, p_{\mu}, p^{*}\right) \delta\left[\begin{array}{c}
q_{E}+q_{\mu}, q^{*} \\
n_{E}+n_{\mu}, n^{*}
\end{array}\right]}
\end{aligned}
$$

where $G_{n}^{\prime}=G_{n}+k_{z}$ and the formulation requires two sets of field related indices (qpn) and $(q p n)^{*}$. The block elements are collected for the field indices constant along a row and the orthogonal indices constant down a column. The indices $(q p n)^{*}$ relate to applying the complex conjugate before the integration of the cylindrical domain. The orthogonal properties of the azimuthal and axial coordinates are preserved and lead to the requirement: $q_{E}+q_{\mu}=q^{*}$ for the azimuthal indices and $n_{E}+n_{\mu}=n^{*}$ for the axial indices. These conditions arise from the orthogonality conditions of the exponential functions. The delta 
function determines the value of the azimuthal index, $q$, which provides a non-zero field expansion coefficient, $\delta_{q_{E}+q_{\mu}, q^{*}}$.

Within these expressions, $\left(S_{1}, T_{o}, T_{1}, U_{1}, V\right)$ are integrals over the radial extent of the computation domain. The integrals are evaluated over the normalized range $x=\frac{r}{R}(0$ to 1) and involve various combinations of zero- and first-order Bessel functions. The orthogonality conditions do not apply to the Bessel functions because multiplication of the integrating term results in a product of three Bessel functions. These integrals are provided in Appendix B. An individual element of the block can be generated using these integral expressions. Since the integrals are independent of the dielectric profile and particular field components required, they are only calculated once. They can be used in the steady state determination using the expressions from Appendix B for any dielectric profile where the radial direction is expanded in the lowest-order Bessel series. The matrix has three rows of blocks and three columns of blocks. The block's order is equal to the number of basis functions used to decompose the field components.

A similar theoretical development can be applied to Ampere's law (5.8-right), which will generate a matrix system similar to that of (5.17) using the symbol change ( $\mu \Rightarrow$ $\varepsilon, E \Rightarrow \mathfrak{J})$, see (5.20) below. This greatly simplifies the computation and programming procedure as Ampere's and Faraday's laws can be treated using the same numerical algorithms. 


$$
\begin{aligned}
& {\left[\mathfrak{I}_{\text {block }}\right]}
\end{aligned}
$$

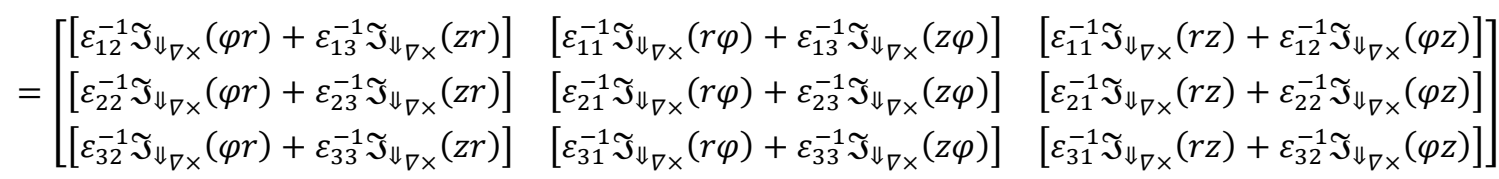

For Ampere's law, the matrix element notation is:

$$
\begin{aligned}
& {\left[\varepsilon_{i 2}^{-1} \mathfrak{J}_{\Downarrow_{\nabla \times}}(\varphi r)+\varepsilon_{i 3}^{-1} \mathfrak{I}_{\Downarrow_{\nabla \times}}(z r)\right]=\mathfrak{I}_{\mathrm{i} 1}(1)+\mathfrak{I}_{\mathrm{i} 1}(2)} \\
& {\left[\varepsilon_{i 1}^{-1} \mathfrak{J}_{\Downarrow_{\nabla \times}}(r \varphi)+\varepsilon_{i 3}^{-1} \mathfrak{I}_{\Downarrow_{\nabla \times}}(z \varphi)\right]=\mathfrak{J}_{\mathrm{i} 2}(1)+\mathfrak{J}_{\mathrm{i} 2}(2)} \\
& {\left[\varepsilon_{i 1}^{-1} \mathfrak{J}_{\Downarrow_{\nabla \times}}(r z)+\varepsilon_{i 2}^{-1} \mathfrak{I}_{\Downarrow_{\nabla \times}}(\varphi z)\right]=\mathfrak{I}_{\mathrm{i} 3}(1)+\mathfrak{I}_{\mathrm{i} 3}(2)}
\end{aligned}
$$

Equation (5.18) can be collected to the matrix system suitable for determining the electric field components and angular frequencies. The system matrix results from the three field components written as three separate equations collected in the following overall form:

$$
\left[E_{\text {block }}\right]\left[\begin{array}{c}
\kappa_{E r} \\
\kappa_{E \varphi} \\
\kappa_{E z}
\end{array}\right]=\left(\frac{\omega}{c}\right)\left[\begin{array}{c}
\kappa_{\Im} \\
\kappa_{\Im \varphi} \\
\kappa_{\Im z}
\end{array}\right]
$$

This left-side square matrix has a radial equation along the first row, angular field along the second row, and azimuthal field along the third row. The subscript indicates the row designation of the orthogonal field component. Similarly, magnetic field components (5.20) can form a matrix system: 


$$
\left[\widetilde{I}_{\text {block }}\right]\left[\begin{array}{l}
\kappa_{\Im r} \\
\kappa_{\Im \varphi} \\
\kappa_{\Im z}
\end{array}\right]=\left(\frac{\omega}{c}\right)\left[\begin{array}{c}
\kappa_{E r} \\
\kappa_{E \varphi} \\
\kappa_{E z}
\end{array}\right]
$$

The matrices in (5.22) and (5.23) can be used to uncouple the fields, and for the electric field equation gives:

$$
\left[\widetilde{J}_{\text {block }}\right]\left[E_{\text {block }}\right]\left[\begin{array}{l}
\kappa_{E r} \\
\kappa_{E \varphi} \\
\kappa_{E z}
\end{array}\right]=\left(\frac{\omega}{c}\right)^{2}\left[\begin{array}{c}
\kappa_{E r} \\
\kappa_{E \varphi} \\
\kappa_{E z}
\end{array}\right]
$$

Likewise, the magnetic field components and angular frequencies can be determined by interchanging the order of the block term multiplication:

$$
\left[E_{\text {block }}\right]\left[\widetilde{\Im}_{\text {block }}\right]\left[\begin{array}{l}
\kappa_{\mathfrak{I} r} \\
\kappa_{\Im \varphi} \\
\kappa_{\mathfrak{\Im} z}
\end{array}\right]=\left(\frac{\omega}{c}\right)^{2}\left[\begin{array}{c}
\kappa_{\Im r} \\
\kappa_{\Im \varphi} \\
\kappa_{\mathfrak{I} z}
\end{array}\right]
$$

Either of the last two equations can be solved for field components. The missing other field components can be obtained through the matrix form of Faraday's or Ampere's law (5.8). The choice depends on which field component is to be obtained (5.24) for $\vec{E}$ or (5.25) for $\overrightarrow{\mathfrak{I}}$.

\subsubsection{Full Order Field Formulation}

Another form of the matrix system is available, which solves a 6-field component (5.26) eigenvector with Faraday's and Ampere's matrix operator block being the antidiagonal matrix blocks [91]. The curl expressions in (5.24) and (5.25) can be combined into a single matrix system by forming an extended field vector composed of six 
components. Three from the electric field and three from the scaled complex magnetic field. The resulting system matrix is expressed as:

$$
\left[\begin{array}{cc}
0 & {\left[E_{\text {block }}\right.}
\end{array}\right]\left[\begin{array}{l}
\overrightarrow{\kappa_{E}} \\
{\left[\widetilde{\Im}_{\text {block }}\right.}
\end{array}\right]=I\left(\frac{\omega}{c}\right)\left[\begin{array}{l}
\overrightarrow{\kappa_{E}} \\
\overrightarrow{\kappa_{\mathfrak{J}}}
\end{array}\right]
$$

The solvable system's order is now equal to six times the number of basis functions required to specify the field components' series expansions. The order of (5.26) is twice what is obtained from (5.24) or (5.25). The matrix populating process is straightforward due to the large number of zero blocks in the matrix, 22 out of 36 blocks. The solution provides eigenvalues, which are the states' complex angular frequency divided by $c$. The doubling the size of the matrix order makes this reformation of the FFB technique unattractive when a large number of basis functions are utilized in the series expansions.

From (5.24) and (5.25), it is seen that eigenvalues are in a set of $\left(\frac{\omega}{c}\right)^{2}$ and determined by diagonalizing the larger matrix. It contains information on both the real and imaginary components of the angular frequency. The eigenvalues can be written in complex form as:

$$
\left(\frac{\omega}{c}\right)^{2}=W_{\text {real }}+j W_{i m g}
$$

The angular frequencies can be obtained from the eigenvalues through the following:

$$
\begin{aligned}
& \omega_{\text {real }}^{2}-\omega_{i m g}^{2}=c^{2} W_{\text {real }} \\
& 2 \omega_{\text {real }} \omega_{i m g}=c^{2} W_{i m g}
\end{aligned}
$$

When (5.28) and (5.29) are inverted, it gives the field's complex angular frequency: 


$$
\begin{aligned}
& \omega_{\text {img }}=\frac{c}{\sqrt{2}} \sqrt{-W_{\text {real }} \pm \sqrt{W_{\text {real }}^{2}+W_{i m g}^{2}}} \\
& \omega_{\text {real }}=\frac{c}{\sqrt{2}} \sqrt{W_{\text {real }} \pm \sqrt{W_{\text {real }}^{2}+W_{\text {img }}^{2}}}
\end{aligned}
$$

The eigenvectors represent the expansion coefficients of the field profile and may also contain real and complex contributions. These are obtained at the same time as the eigenvalues using the eig () function in MATLAB $C^{\circ}$. Examining the eigenvector coefficients and determining the dominant field contribution provides insight into the state's properties.

\subsubsection{Matrix Order Reduction}

The orthogonal integration substantially reduces the Eigen matrix. This integration restricts the azimuthal indices combinations acquired for non-zero values of the matrix elements. When: 1$)$ the material profile shows no $\varphi$ variations, then at $q_{\varepsilon}=0$, the pairs $\left(q, q^{*}\right)$ must be equal to the non-zero matrix elements; 2$)$ The mixing of angular indices for the material and field satisfies the relation $q_{f}+q_{m}=q_{f}^{*}$ where $m$ indicates permittivity or permeability. Combining these two conditions implies that the field indices difference corresponds to an integer multiple of the rotational symmetry of the material present. For instance, in a dielectric structure that possesses $\mathrm{N}$-fold rotational symmetry in $\varphi$, the non-zero expansion coefficients for the permittivity are dictated by the angular indices $\left(q_{\varepsilon}=\cdots,-2 N,-N, 0,+N,+2 N, \ldots\right)$. For a state which contains $q_{f}=0$ expansion 
coefficient in its representation corresponds to the monopole family and may contain the non-zero expansion for $\left(q_{f}=\cdots,-2 N,-N, 0,+N,+2 N, \ldots\right)$. For all other $q_{f}$ values the monopole family has a zero-expansion coefficient due to the mixing condition $\left(q_{f}+q_{m}=\right.$ $q_{f}^{*}$. The state which contains $q_{f}= \pm 1$ expansion coefficient in its representation corresponds to dipole family and may contain non-zero expansion coefficients $\left(q_{f}= \pm 1\right.$, $\pm N \pm 1, \pm 2 N \pm 1, \ldots)$. The mixing condition results in two separate sets of coefficients, one set by selecting the ",$+ \mathrm{CW}$ " $\operatorname{sign}\left(q_{f}=+1, \pm N+1, \pm 2 N+1, \ldots\right)$ and one by selecting the ",$- \mathrm{CCW}$ " sign $\left(q_{f}=-1, \pm N-1, \pm 2 N-1, \ldots\right)$. The standing wave providing steady state conditions is obtained by the superposition of the two counterpropagating fields. This is obtained when the "+" and the "-" components are combined. The eigenvalues obtained using the set "+" are the complex conjugate of the eigenvalue obtained using the "-". Thus, only one of them must be solved. Within the matrix structure, each mode family has its column and rows padded with zeroes, and as a result, each mode family matrix can be extracted and solved individually. Thus, mode family tuning can reduce the system matrix's order to solve the material's rotational symmetry. This frees up computer resources and provides computed results sooner.

\subsubsection{Perfectly Matched Layer}

An absorbing layer may accommodate the introduction of a PML at the computational domain external perimeter. The discussion regarding PML and $\mathrm{ABC}$ from the FDTD Section, 5.2.2, can be imported to FFB. In the cylindrical computational 
domain, absorbing regions are required for the radial $(r)$ and axial $(z)$ directions. The absorbing material matrix is expressed as:

$$
\begin{aligned}
& \overleftrightarrow{s}=\left[\begin{array}{ccc}
s_{r}^{-1} & 0 & 0 \\
0 & s_{r} & 0 \\
0 & 0 & s_{r}
\end{array}\right]\left[\begin{array}{ccc}
s_{z} & 0 & 0 \\
0 & s_{z} & 0 \\
0 & 0 & s_{z}^{-1}
\end{array}\right] \\
& \overleftrightarrow{s}=\left[\begin{array}{ccc}
s_{r}^{-1} s_{z} & 0 & 0 \\
0 & s_{r} S_{z} & 0 \\
0 & 0 & s_{r} s_{z}^{-1}
\end{array}\right]
\end{aligned}
$$

where $s_{r}=\kappa_{r}-j \frac{\sigma_{x}}{\omega \varepsilon_{o}}$ and $s_{z}=\kappa_{z}-j \frac{\sigma_{z}}{\omega \varepsilon_{o}}$. With $s_{r, z}=s_{r_{r, z}}-j s_{i_{r, z}}$ conforming to the theoretical development proposed here. For the radial PML direction $s_{z}=1$, the PML is placed at the upper and lower extremes of the axial computation direction, increasing from zero to a maximum at the upper and lower edges following a power profile and in the axial direction $s_{r}=1$. In the corner regions, both PML directions are required in (5.33). The PML layer could also be placed in the free space region making $s_{r_{r, z}}=1$.

The PML region bordering the computational domain will introduce loss into the structure in much the same way that the PML introduces loss in the FDTD algorithm. The PML placed sufficiently far away from the resonator structure ensures that resonator state field components (those we seek using the FFB technique) are reduced to zero at the computational domain edge. The field component series in (5.11) and (5.12) are zero at the computational edge due to the argument of the Bessel functions used in the basis functions. The introduction of the PML ensures that the series (5.11) and (5.12) represent realistic 
fields with open-ended boundary conditions as the PML forces the field component to be zero at the radial extreme. The FFB version of the PML will be included when numerically examining the states of the proposed MBRs.

A parameter desired when designing a resonator is the Quality factor, $Q$. The dielectric MBR structure under theoretical analysis in this thesis has no loss mechanisms presented. Thus, the eigenfrequencies of the bottle states are real and indicate an infinite $Q$. However, in an actual application of the bottle resonator, input and output power coupling mechanisms must be included, and the $Q$ of the resonator would be finite. An estimation of the $Q$ factor is possible by taking the field profile of the bottle confined mode of interest and calculating its overlap.

The FSR can be found in two ways: wavelength spacing of similar states with adjacent azimuthal order or wavelength spacing of contiguous mode within an azimuthal order.

The numerical solver used for computations in this thesis works on cylindrical coordinates, provides mode wavelength, field profile and $Q$. It utilizes FFB basis function series expansion of the material and optical fields, dramatically simplified using Faraday's and Ampere's law rather than the usual wave equation. It can include the permittivity (permeability) with real and imaginary parts and may have a PML. This method uses FFB expansions of the field and inverse dielectric to express Maxwell's equations as an eigenvalue problem. The eigenvalues obtained by solving these expressions are the frequencies, which can be converted to free space wavelengths, and the eigenvectors are the FFB expansion coefficients for the field profiles. The FFB solver results agree with 
other numerical methods, such as FDTD and PWEM, when these techniques can be applied to the same structure. In the next chapter, the FFB numerical method examines MBR (solid and hollow) structures as these resonators conform to a cylindrical coordinate system. 


\section{Chapter 6: MBR Computational Profile}

\subsection{Solid Bottle Profile}

The solid glass (BK-7) bottle resonator's geometry under consideration is shown in Figure 6.1-left. The structure is non-magnetic with a relative permeability of 1 . It consists of a high relative permittivity $\left(\varepsilon_{r}=2.3000\right)$ isotropic cylinder of $r=2 \mu \mathrm{m}$ radius and $T=$ $10 \mu \mathrm{m}$ height centered, and aligned with the cylindrical coordinate axis. The ambient medium is air with a relative permittivity of 1.000 . The bottle confinement is accomplished by sculpting two rings about the azimuthal direction into the cylinder. Each ring has an axial extent of $D=2 \mu \mathrm{m}$, follows a cosine profile with an amplitude of $1 \mu \mathrm{m}$, and is located a distance $\mathrm{W}$ evenly above and below the coordinate system's central plane. The radial domain of the computation extends out to $5 \mu m$ with the last $2 \mu m$ configured as a PML to simulate an open-ended resonator. The structure is periodic along the $z$-axis, and Figure 6.1-center shows the structure plotted over three periods. Two bottle regions are obtained. One is centered on the coordinate plane and referred to as the central bottle resonator, $\mathrm{C}$, while the adjacent is referred to as the upper, $\mathrm{U}$, bottle resonator. In principle, the structure's numerical simulation can proceed as a function of all parameters used to define the structure and is the subject of extended work on sensors discussed in Chapter 7. Here the ring placement is changed as well as the ambient medium's refractive index. The former defines the bottle region width, while the latter is used to simulate a sensing environment. 

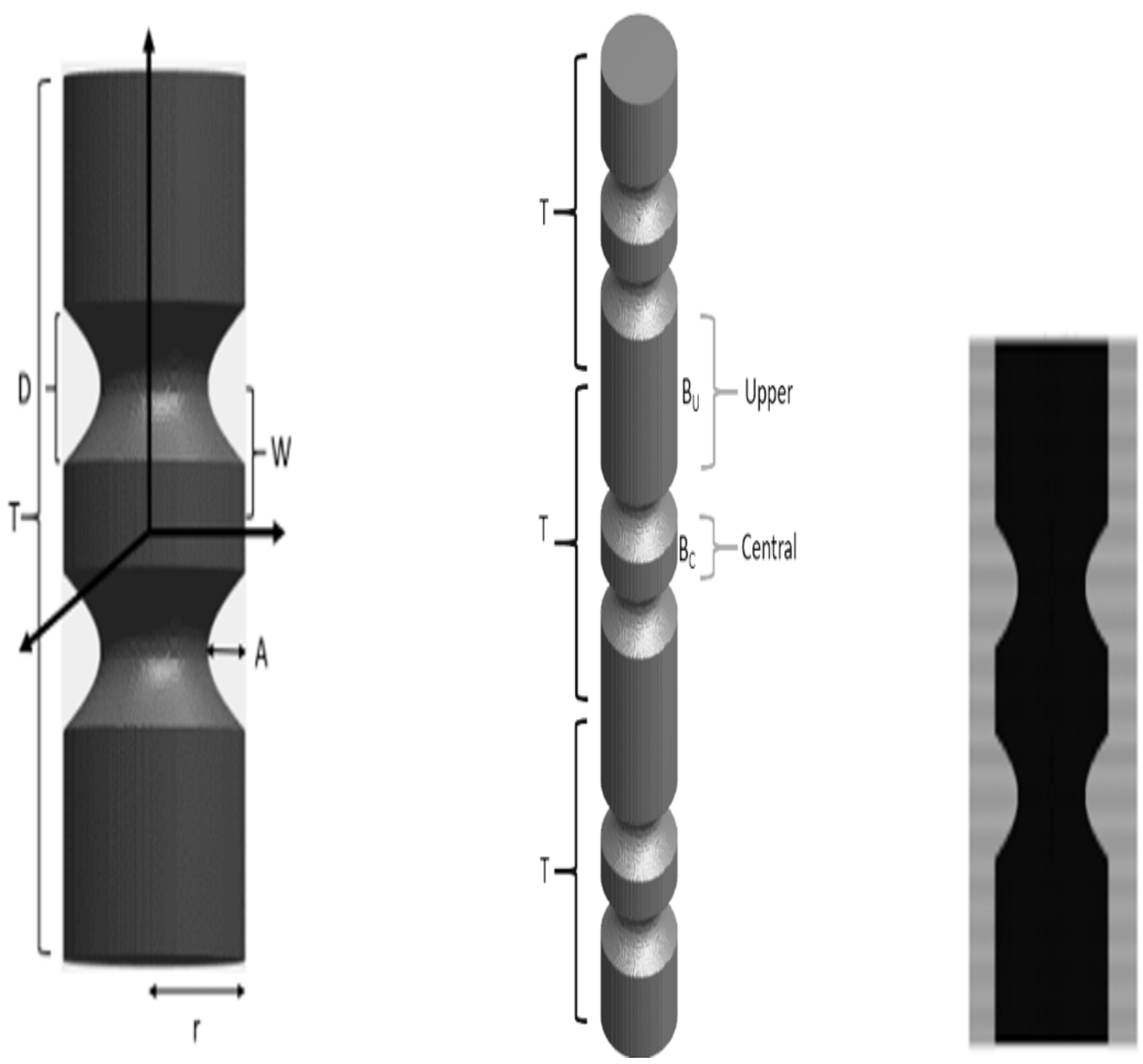

Figure 6.1 Left is the geometry and parameter identification of the MBR used in the numerical computations and sensor configurations. The structure is periodic in the axial direction with three periods shown (center), and uniform under azimuthal rotation. The right figure shows the reconstruction of the $(1,1)$ relative permittivity tensor element showing that the original bottle resonator is obtained and ensures convergence in the spectral decomposition. 
The computation of the bottle resonator states requires that all parameters defined in Figure 6.1 be given numerical values. The relative permittivity and permeability of the structure, ambient medium and PML border are then discretized on a cylindrical grid using 50 points per micron in the radial and axial directions. Since the structure has rotational symmetry about the $z$-axis, only the medium properties at $\varphi=0$ are required. This symmetry converts the 3 -D structure $(r, \varphi, z)$ to one that requires only a 2-D $(r, z)$ analysis when the FFB numerical technique is utilized. The expansion coefficients for the material tensor elements (5.10) are determined through orthogonal integration from the discretized grid. We found that 100 Bessel terms $\left(p_{\varepsilon}=1 \rightarrow 100\right)$ and 199 axial terms $\left(-99 \leq n_{\varepsilon} \leq+99\right)$ with $q_{\varepsilon}=0$ provides an accurate representation, as can be confirmed by the reconstruction of the $\left(\varepsilon_{r r}^{-1}\right)$ elements of the relative permittivity tensor shown in Figure 6.1-right. The dielectric structure is uniform to the azimuthal coordinate $\varphi$ and can thus be regarded as having infinite rotational symmetry. This property implies that the decomposition of the dielectric should be independent of the azimuthal coordinate and imposes the condition that the only non-zero inverse dielectric expansion coefficients are obtained when $q_{\varepsilon}=0$. All non-zero expansion coefficients with axial order other than zero define the bottle dielectric regions. The inverse relative permeability expansion space coefficients must still be computed as the series in (5.10) are required.

When a structure presents a complete rotational symmetry about the $z$-axis, the matrix generated in (5.24) can be separated into much lower order matrices, each specific to a particular field profile azimuthal rotational order. Computations were performed for numerous rotational orders, and the results presented here will focus on the bottle states 
that demonstrate a rotational symmetry of $20, q_{E, \Im}=20$ used in the expansion field series in (5.11) and (5.12). The matrices for azimuthal mode orders 15 to 25 were produced, and the state space was determined and examined. As the azimuthal order is reduced, the wavelength associated with a mode increases and the fields extends into the cylindrical dielectric region. It was observed that the modes with rotational symmetry of 20 were best confined to the bottle region. Numerical tests have shown that the field profiles and corresponding wavelengths for the bottle confined states are sufficiently converged when 50 Bessel terms $\left(p_{E, \Im}=1 \rightarrow 50\right)$ and 89 axial terms $\left(-44 \leq n_{E, \Im} \leq+44\right)$ are utilized. (Note: The convergence is based on examining the variation in the desired bottle states' eigenvalues and eigenvectors.) Some states are confined to the high dielectric cylinder and constitute the desired MBR states. Bottle states are determined by plotting the field profile and examining the field localization properties.

All computations were performed on MATLAB-based algorithms. First, the material profile is proposed in (5.10), and then the expansion space is determined using (5.11) and (5.12). The resonator state properties are obtained by solving the eigenvalue matrix system using the eig () function in MATLAB. The field profiles are obtained from the computational domain using expressions (5.11) to (5.24) as the eig () function returns the eigenvectors for each eigenvalue.

Figure 6.2 shows a space of the modal wavelengths (real part) obtained versus matrix indexing integer, which contains the various radial and axial mode orders for the rotational mode order 20 bottle confined states. This wavelength space is restricted between 
$0.77 \mu \mathrm{m}$ to $0.8 \mu \mathrm{m}$ range to display starting from the upper bottle's mode zero to the central bottle's first-order mode. If the range is extended, it could include many other modes. The

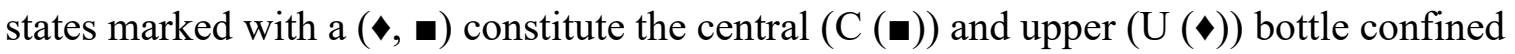
states.

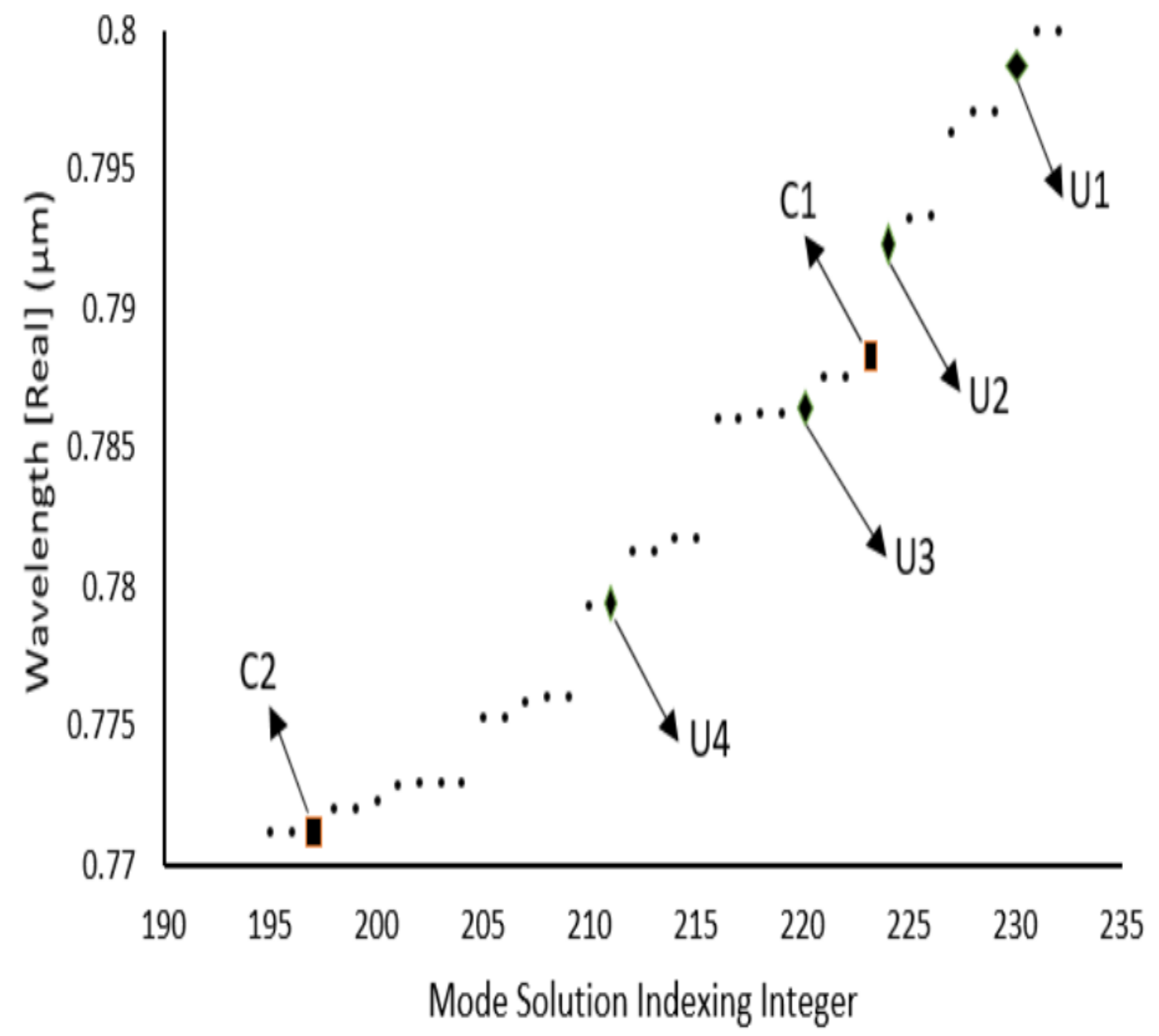

Figure 6.2 The wavelength's real part of computed states versus matrix solution indexing is indicated here. States identified with a (\) correspond to modes highly localized to the high permittivity glass bottle region. 
Figure 6.3 presents the imaginary part of the wavelength for states in the indicated range. Bottle confined states have the imaginary part close to zero-axis as the imaginary contribution in the resonator structure originates from the PML region, where these states have very little field presence. Also, it restricts the expansion space to $q_{\varepsilon}=\{0\}$. The states with azimuthal order differ only in sign, $q=\{+,-\}$ and corresponds to CW and CCW, respectively. The rotational order 20 has $\mathrm{CW}=+20$ and $\mathrm{CCW}=-20$ modes. The eigenvalue space obtained for the pair will be the same, and the eigenvector space will be the complex conjugate of each other. Thus, only one of " + " or " - " can be solved. The matrix +20 is separated from the matrix with -20 . If only the -20 mode order is solved separately and then combined with +20 mode, i.e., superposition of $\mathrm{CW}$ and $\mathrm{CCW}$ states for the same wavelength, it will produce a standing wave viewed as a steady state. Thus, the two modes grouped in the imaginary graph are the degenerate states. This property can be used as an additional filter for determining the bottle confined states. 


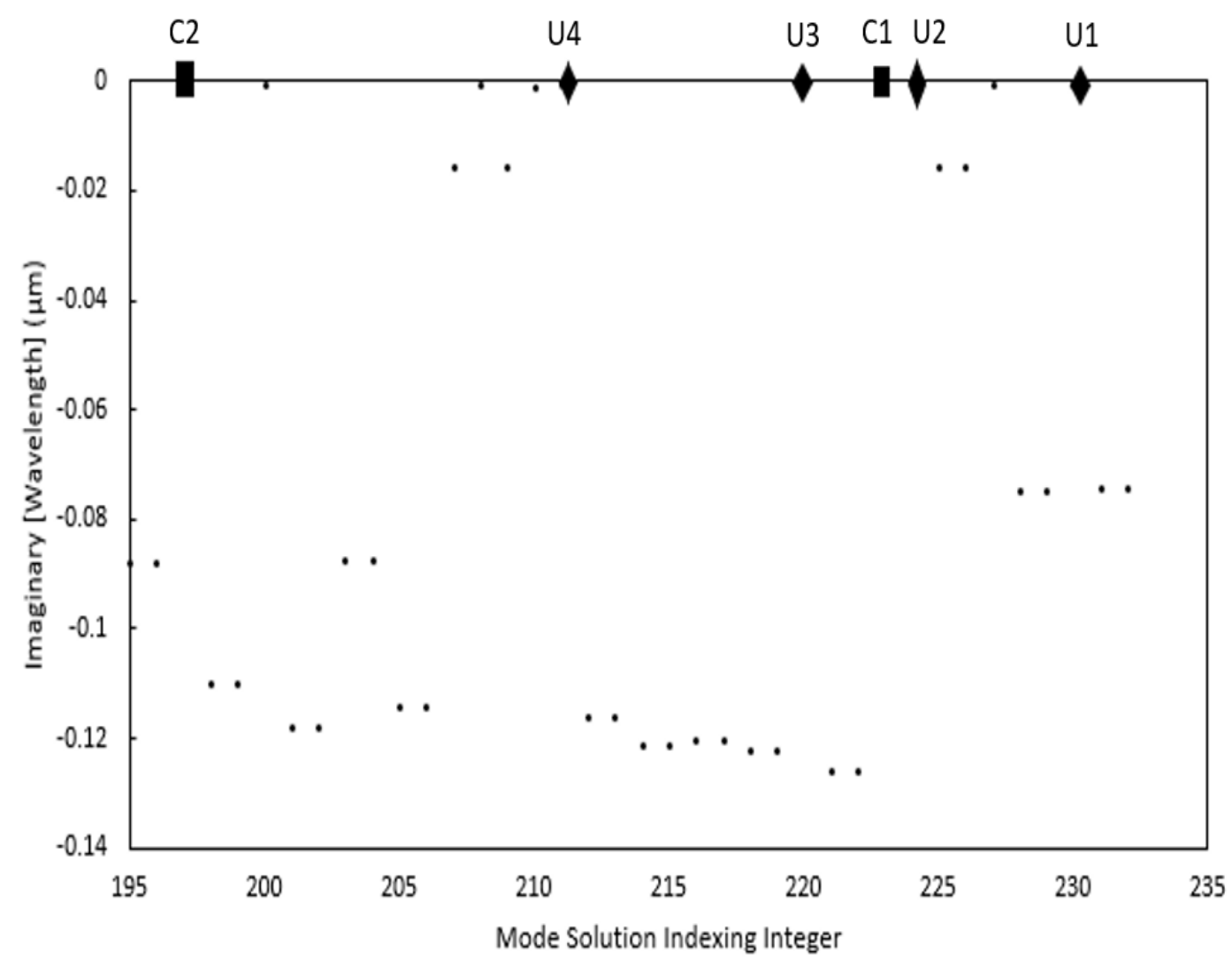

Figure 6.3 The wavelength's imaginary part of the computed states versus matrix solution indexing is indicated here. Bottle confined states have negligible field components in the PML region, making the imaginary part very close to zero.

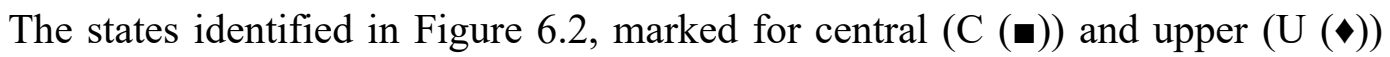
bottle confined states, have field intensity profiles plotted in Figure 6.4. The modes are labelled by the number of 0 crossings in the radial (first integer) and axial (third integer) direction with azimuthal order 20 (middle integer). In addition to the 2-D profiles, the 3-D profiles for the lowest-order mode are shown in Figure 6.5. The intensity profiles display 40 bright maximums per 360-degree rotation as the intensity pulsates twice the mode order of 20 for these states. 


\section{$C 1(0,20,0) \quad C 2(0,20,1) \quad C 3(0,20,2) \quad C 4(0,20,3) \quad C 5(0,20,4)$}
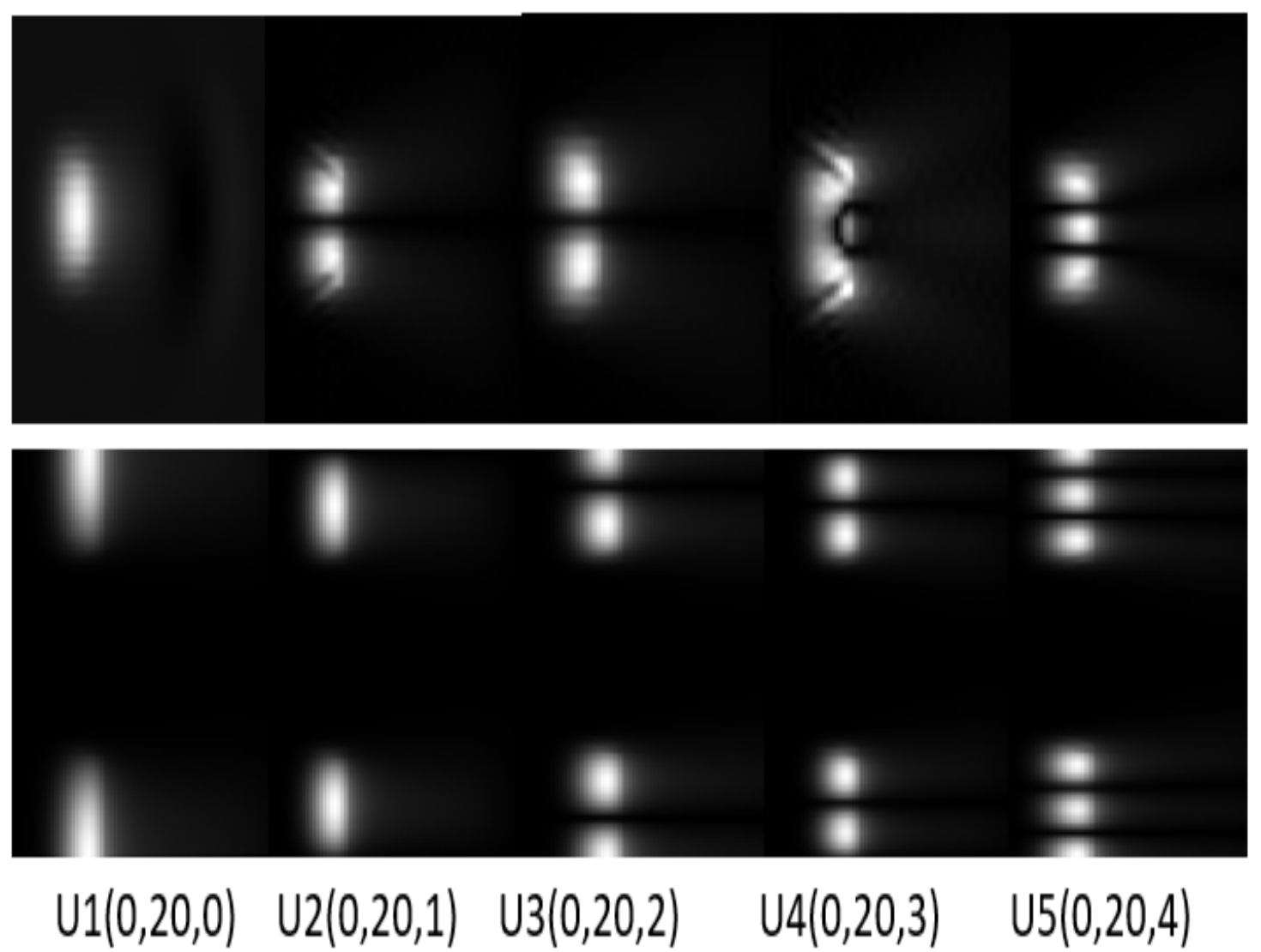

Figure 6.4 The resonator states obtained for the structure in Figure 6.1 at the wavelengths identified in Figure 6.2 when the bottle parameter, $W$, was $1.5 \mu \mathrm{m}$. The top set shows the intensity profile in the $(r, z)$ plane for states localized in the glass cylinder's central region. The bottom set shows the intensity profile for states localized in the upper and lower regions resulting from the additional bottle produced through axial periodicity. 

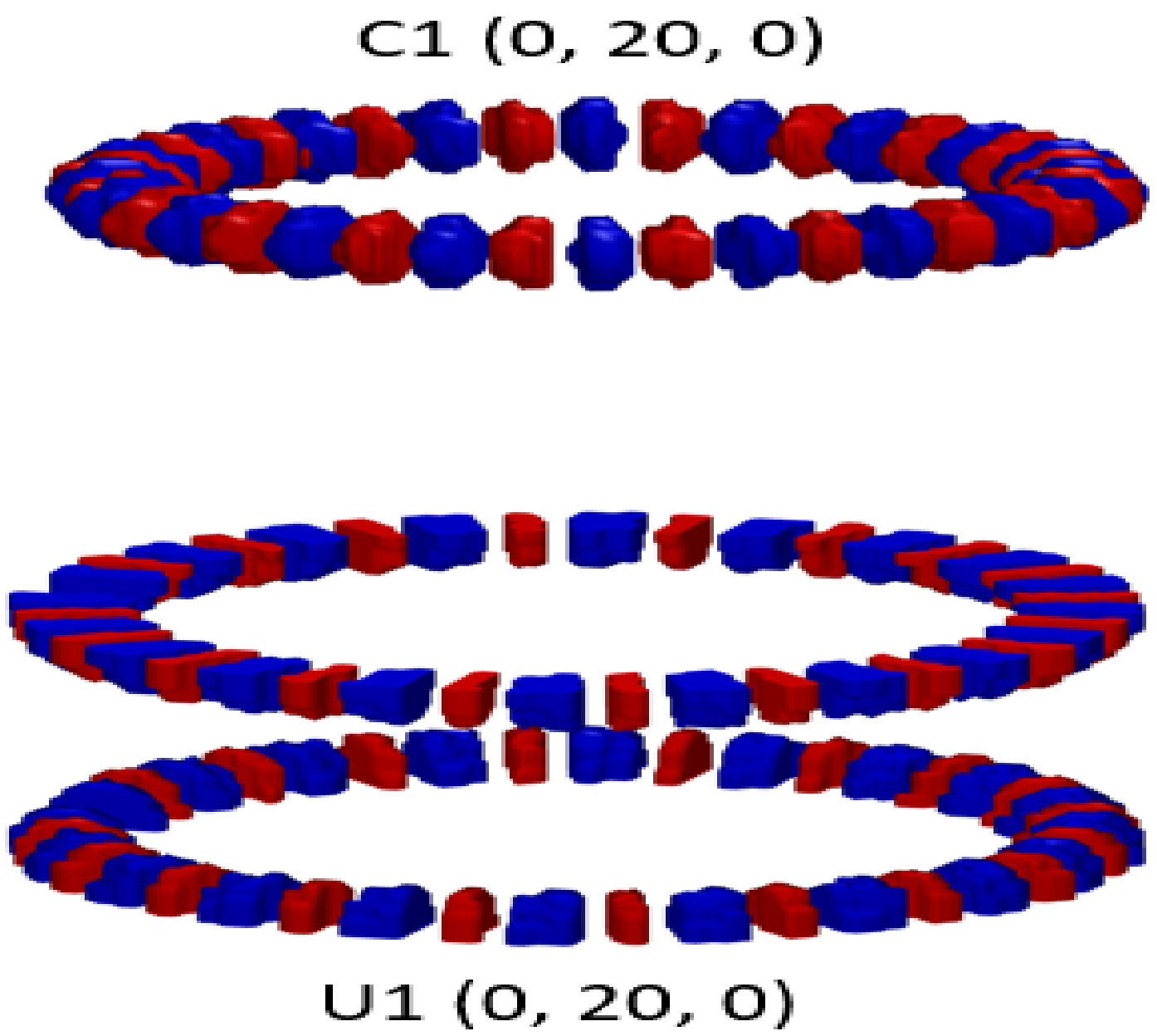

Figure 6.5 The lowest order mode in 3-D representation demonstrating the azimuthal mode order of 20 (40 intensity maximum over $2 \pi$ rotation).

Figure 6.6 shows the lowest-order central and upper states' wavelength variation as a function of ring axial offset, $W$. The central region bottle width can be determined from $\mathrm{B}_{\mathrm{C}}=2 W-D$, and the upper bottle width is determined from $\mathrm{B}_{\mathrm{U}}=T-(2 W+D)$. As expected, the narrower the bottle, the smaller the state's wavelength as the state must fit into the smaller high relative permittivity region. At $W=2.5 \mu \mathrm{m}$, the central and upper bottles are identical and support identical states. The bottle structure with $W=1.5 \mu \mathrm{m}$ is 
selected as the structure to explore as an index of refraction sensor since the central and upper lowest-order states have a sizeable wavelength difference for the chosen structural parameter. $W=1.25 \mu \mathrm{m}$ is not used as it makes the bottle region very narrow. If the confined mode's region is narrow, it may cause the mode to leak out of the bottle into the ring indentations, which is not desired for sensor-designing applied to this thesis.

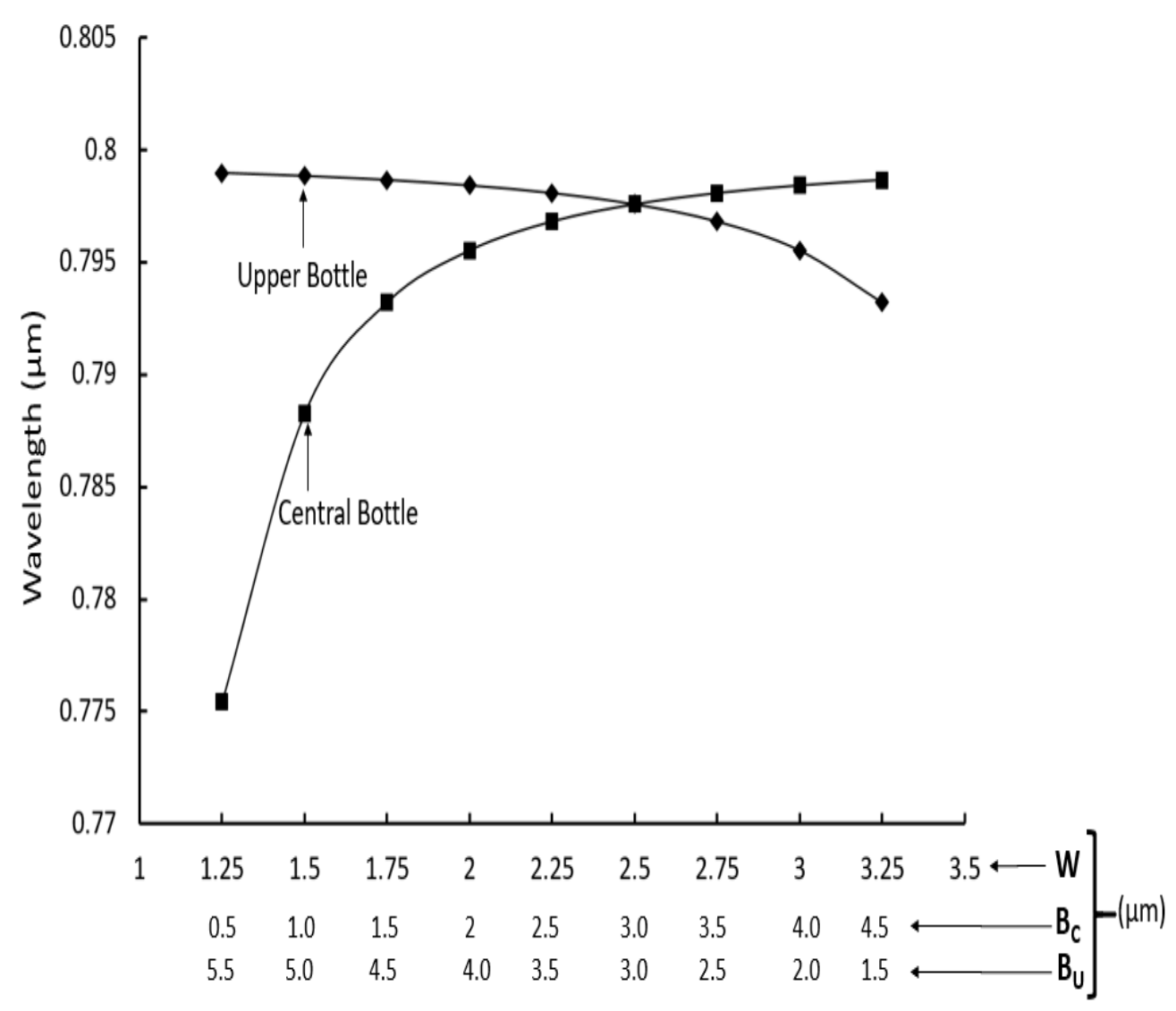

Figure 6.6 Plot of the bottle confined resonator states as a function of the resonator width, $W$ is displayed. At $W=2.5 \mu \mathrm{m}$, the central and upper bottles are identical. For $W<2.5 \mu \mathrm{m}$, the central bottle is narrower than the upper bottle making the lower confined states exhibit a smaller wavelength. The structure with $W=1.5 \mu \mathrm{m}$ was selected for sensor consideration. 


\subsection{Hollow Bottle Profile}

The air-filled hollow glass MBR is presented in Figure 6.7. The material parameters of the solid bottle profile are utilized for this structure. The hollow region is an air $\left(\varepsilon_{r}=\right.$ 1.000) cylinder with a radius of one micron and a height of ten microns. A hollow notch is then created to have a radius of $1.75 \mu \mathrm{m}$ and a height of $1 \mu \mathrm{m}$. This makes the overall hollow structure exhibit thin walls of $0.25 \mu \mathrm{m}$, as discussed in Section 3.3. Each ring has an axial extent of $D=2 \mu \mathrm{m}$, follows a cosine profile of an amplitude $1 \mu \mathrm{m}$, and is located at a distance, $W=1.5 \mu \mathrm{m}$, above and below the central plane of the coordinate system. The radial domain of the computation extends out to $5 \mu \mathrm{m}$ with the last $2 \mu \mathrm{m}$ configured as a PML to simulate an open-ended resonator. This new structure is also centered and aligned with the cylindrical coordinate axis. It is periodic along the $z$-axis. The ambient medium is also air. The hollow and ambient medium's relative permittivity were varied to analyze the hollowed notch's confined mode. 


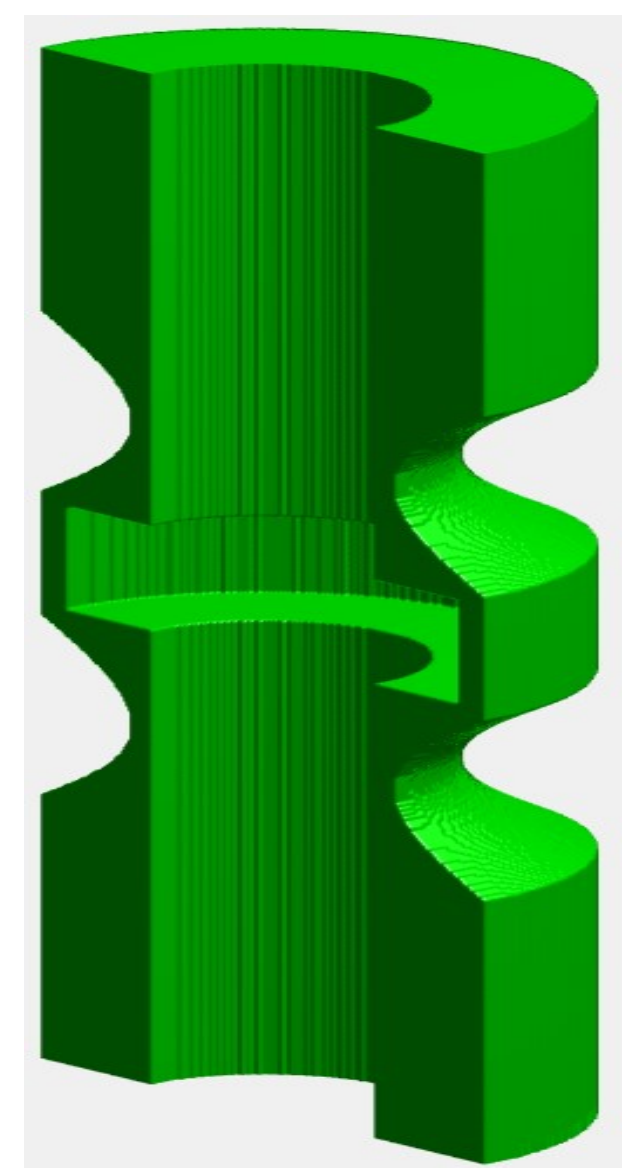

Figure 6.7 The basic geometry of the hollow micro-optic bottle resonator where an additional notch was created inside the central bottle region. The distance of rings from the center is $W=1.5 \mu \mathrm{m}$ with a cosine profile.

The hollow bottle states are determined by plotting the field profile and examining the field localization properties. This is done the same way as simulated in the solid bottle by utilizing the Bessel terms $\left(p_{\varepsilon}=1 \rightarrow 100\right)$ and 199 axial terms $\left(-99 \leq n_{\varepsilon} \leq+99\right)$. The selected field intensity profiles are plotted in Figure 6.8 and constitute either central (C) or upper (U) bottle confined states. The modes are labelled by the number of zero crossings in the radial (first integer) and axial (third integer) direction with an azimuthal order of 20 (middle integer). The intensity profile for the upper bottle region is the same as 
that of the solid bottle, whereas in the air-filled hollow notch the field intensity is confined to the bottle's thin walls.

\section{$\mathrm{C} 1(0,20,0) \quad \mathrm{C} 2(0,20,1) \quad \mathrm{C} 3(0,20,2)$}
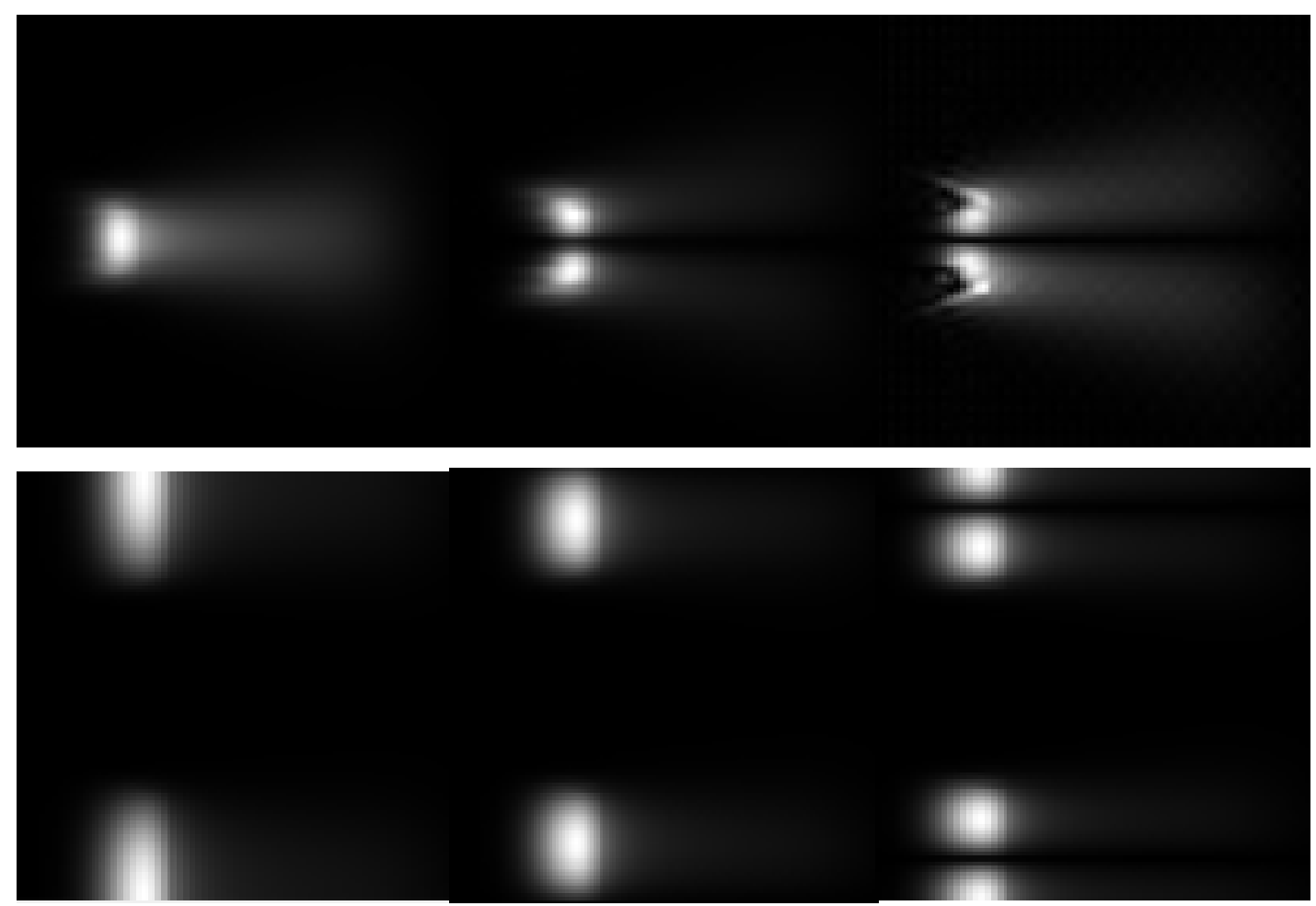

\section{$\mathrm{U} 1(0,20,0) \quad \mathrm{U} 2(0,20,1) \quad \mathrm{U} 3(0,20,2)$}

Figure 6.8 The resonator states obtained for the structure of Figure 6.7 at the wavelengths

identified in Figure 6.9 when the bottle parameter, $W$, is $1.5 \mu \mathrm{m}$. The top set shows the intensity profile in the $(r, z)$ plane for states localized in the central bottle region's thin-walls. The bottom set shows the intensity profile for states localized in the upper and lower regions resulting from the additional bottle produced through axial periodicity. 
The computed wavelength (real or imaginary part) helps identify the mode profile easily, which is to be constructed. Figure 6.9 displays the real part of the wavelength for the HMBR's computed states. The wavelength space is restricted between $0.71 \mu \mathrm{m}$ and $0.81 \mu m$ to display starting from the upper HMBR's mode zero to the central HMBR's second-order mode. The graph shows that the modes are either in a pair of two or single. The modes of interest are confined in the central and upper HMBR regions and can be easily identified as a single-mode. These HMBR's localized states are marked with a (C (๘), U (४)) on the graph for the wavelength identification. The wavelengths for HMBR's upper region field intensity profile are $\mathrm{U} 1=0.7988 \mu \mathrm{m}, \mathrm{U} 2=0.7963 \mu \mathrm{m}$, and $\mathrm{U} 3=$ $0.7921 \mu \mathrm{m}$. The wavelengths for the central bottle's field intensity profile are $\mathrm{C} 1=$ $0.7533 \mu \mathrm{m}, \mathrm{C} 2=0.7264 \mu \mathrm{m}$, and $\mathrm{C} 3=0.7140 \mu \mathrm{m}$.

The imaginary part of the wavelength further helps to easily distinguish between the state localized in the upper and central HMBR's region. Figure 6.10 illustrates the imaginary part of the wavelength of the HMBR's computed states. The MBR's imaginary graph (see Figure 6.3) is slightly different from the HMBR's. The difference is due to the addition of a hollowed notch into the central MBR's region. The states localized in the HMBR's thin-walled central region are below the zero-axis and identified by a ( $\mathbf{})$ symbol. They appear to be as single states among other degenerate states. The single states localized on the zero-axis (top right corner) are the HMBR's upper region represented by the ( $)$ symbol. The rest of the states on the zero-axis are the higher-order modes of the HMBR's upper region. The lowest-order mode localized in the HMBR's thin-walled central region can serve as WGM resonance utilized for sensor detection. 


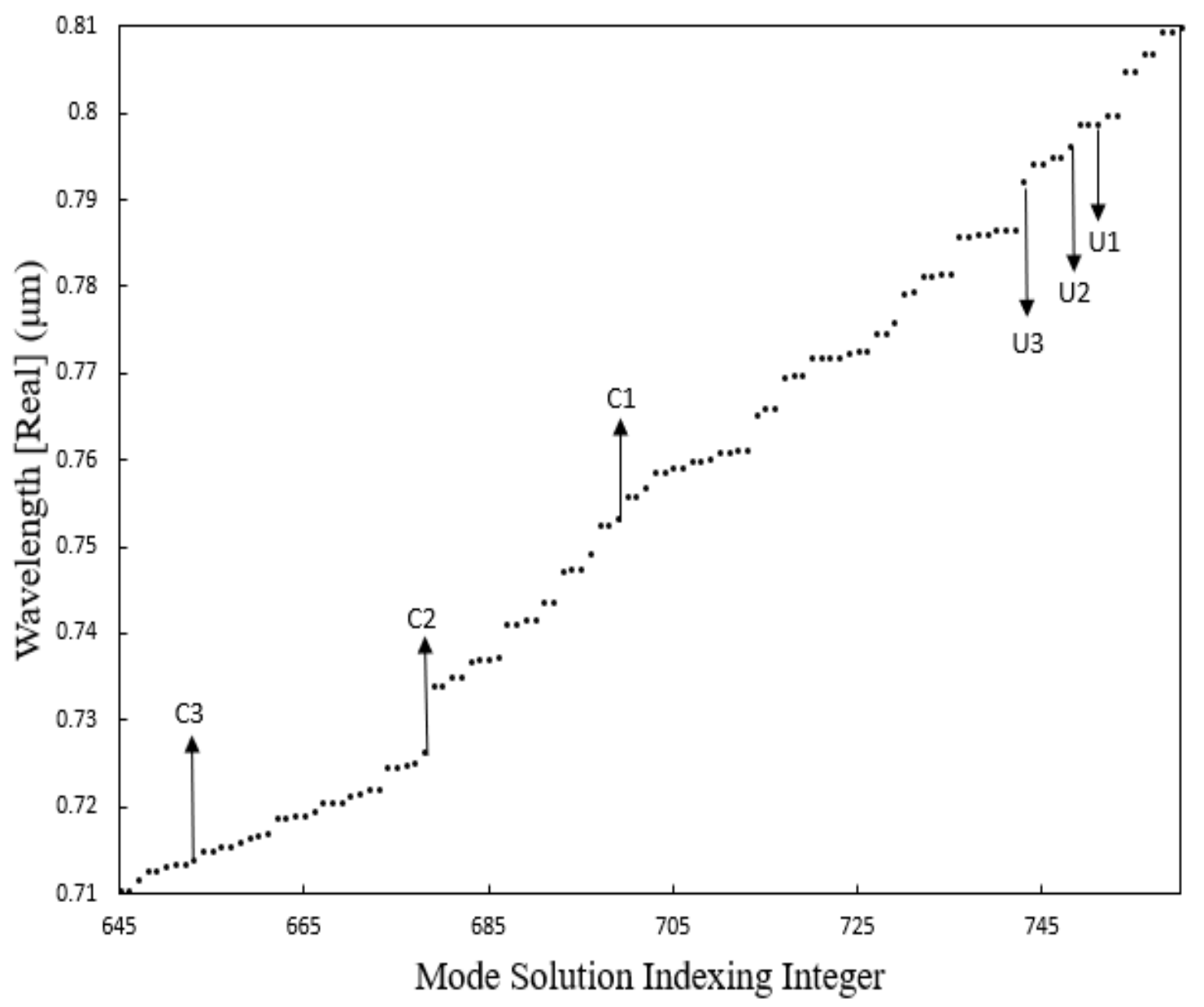

Figure 6.9 The real part of the wavelength for the HMBR's computed states versus matrix solution indexing. States identified with a (•a) correspond to modes highly localized to the bottle region with a high permittivity glass cylinder. 


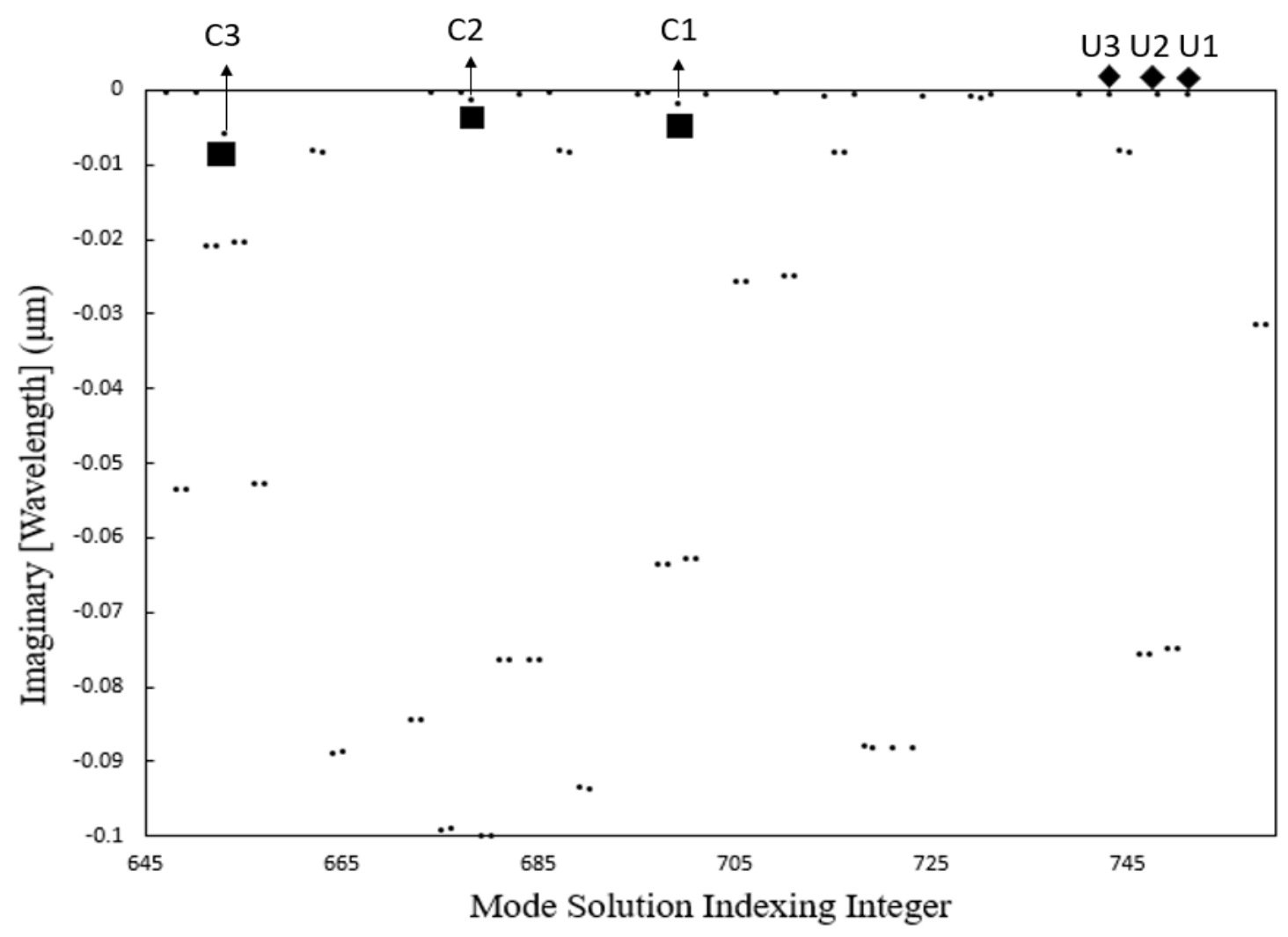

Figure 6.10 The imaginary part of the wavelength for the HMBR's confined states has negligible field components in the PML region making the imaginary part very close to zero.

This chapter presents the bottle resonators' geometrical and material properties and information on the parameters selected to ensure convergence for the eigenstates returned in the numerical computations. The modal space for the MBR as a function of axial width of the central bottle spacing is provided, and samples of the available modes are plotted. Later in this chapter, HMBR is built to a fixed axial width spacing. The modal properties of MBR and HMBR have the potential to be used as sensor applications. The sensors configured for the MBR and HMBR are presented in the next chapter. 


\section{Chapter 7: MBR Sensor Configurations}

This chapter presents the theoretical results obtained from the MBR proposed in Chapter 6, operating as an optical sensor. All results are computed using the FFB numerical mode solver. The MBR will be simulated for a refractive index/gas, temperature, and chemical sensor.

The MBR is first examined as a refractive index/gas sensor where the change in the ambient region's refractive index is detected. A sensor simulation is performed where the presence of $\mathrm{CO}_{2}$ gas in the ambient medium is to be sensed.

The performance of the MBR structure acting as a temperature sensor is simulated. Temperature changes manifest themselves through the thermo-optic effect and thermal expansion of the MBR. The MBR's temperature-dependent sensitivity is calculated through a wavelength shift caused by the refractive index change and thermal expansion. It is known that Maxwell's equations are size scalable, so the calculated effects in linear dimensions will be included in the wavelength shifts computed for the thermo-optic effect taken alone. For $\mathrm{CO}_{2}$ gas as the ambient medium, temperature changes in the gas and structure are computed. As the temperature changes will affect the resonator and gas properties, the overall shift in wavelength is recorded to be used in the sensor's sensitivity calculations.

In a chemical sensor, the MBR's central bottle region is hollowed-out along its axis to create a thin-wall HMBR. When the chemical flows through its internal and external surroundings medium, changes in the resonance mode's wavelength are calculated. The 
wavelength shift due to a change in refractive index is used in the sensitivity estimation. The concentrated molar volume of chemicals in liquid form, i.e., benzene and chlorobenzene, are to be detected using a flow through in the HMBR's internal region.

\subsection{Refractive Index Sensing}

Herein, the ability to measure the ambient medium's refractive index (relative permittivity) through a wavelength shift of the lowest-order state axially directed in the central and upper region of the MBR for mode 20 in the azimuthal direction is examined. The central MBR's axial width is set at $W=1.5 \mu \mathrm{m}$ giving the structure shown in Figure 6.1. Each new structure's geometry is discretized with 50 grid points per micron to determine the series expansion space for the components of the relative permittivity tensor. The states are then computed using 50 Bessel terms and 89 axial terms for azimuthal mode order $20(q=20,1 \leq p \leq 50,-40 \leq n \leq+40)$. The wavelength and its shift are tracked by plotting the states returned from each computation and identifying the lowest-order state profiles (axially directed) confined in the bottle (central and upper region). The wavelength as a function of the ambient medium's refractive index (relative permittivity) is plotted in Figure 7.1. In general, the refractive index closest to 1.0 would monitor airborne impurities and trace gases in the ambient medium to emulate the gaseous environment. The sensitivity

is determined using Eq. (4.1) as $S=\frac{\Delta \lambda}{\Delta n}$. The sensitivity in the gaseous region (indicated in the graph) from the refractive index is 1 to 1.0723 , and using the central bottle's lowestorder mode wavelength is $86.9 \frac{\mathrm{nm}}{\mathrm{RIU}}$. It was also seen in the graph that a refractive index from 1.0723 to 1.1269 is suitable for intermediate materials (such as blood, osmium and 
calcium fluoride). A refractive index from 1.1269 to 1.2041 can be used for sensing various liquids. The central bottle's lowest-order mode wavelength is calculated over the liquid zone using the refractive index range mentioned in the graph; the sensitivity in this zone is $\mathrm{S}=\frac{\Delta \lambda}{\Delta n}=123.6 \frac{\mathrm{nm}}{R I U}$. A refractive index from 1.2041 to 1.2247 represents solid materials, and these values are used for sensitivity detection in this region using the central bottle's lowest-order mode wavelength as $S=\frac{\Delta \lambda}{\Delta n}=125.9 \frac{n m}{R I}$. Similar results were extracted for three zones (gas, liquid, and solid) using the upper bottle's lowest-order mode (axially directed) wavelength. Table 7.1 has the refractive indices and corresponding computed wavelength for both the central and upper bottle regions. Table 7.2 has the calculated sensitivity extracted from each zone for the upper bottle region. There is a noticeable difference in solid zone sensitivity for the central and upper bottle region. This is due to the wavelength drops in the upper bottle region from 1.20 to 1.23 refractive index range, as seen in Figure 7.1. 


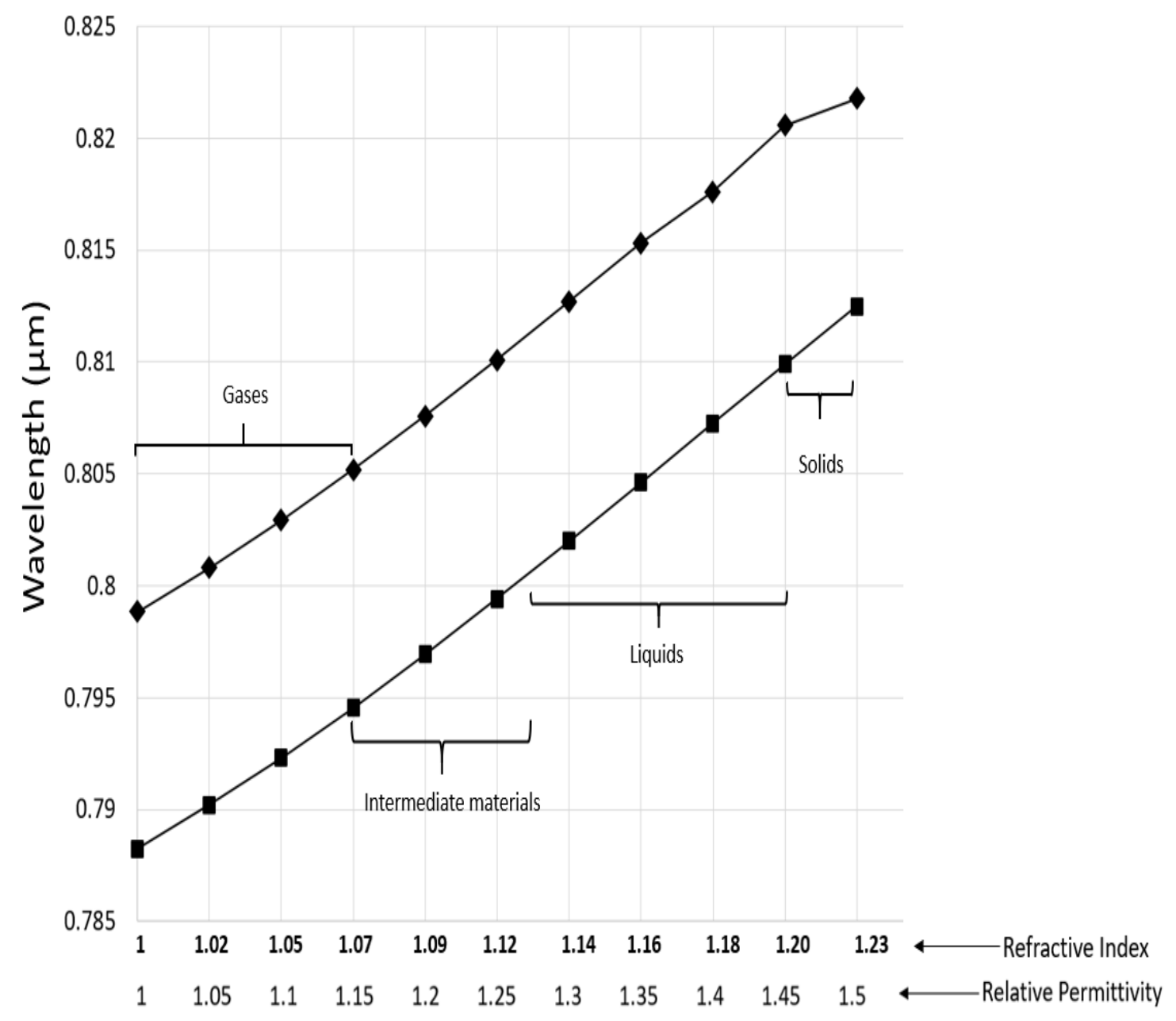

Figure 7.1 The bottle confined mode wavelength response as a function of the ambient medium's index of refraction / relative permittivity for the lowest-order mode axially directed in the upper bottle. It is denoted by ( $\bullet$ in the central bottle region and (ロ) for azimuthal order 20. 
Table 7.1 The refractive index and its corresponding mode zero wavelengths for the central and upper bottle regions.

\begin{tabular}{|c|c|c|}
\hline $\begin{array}{c}\text { Refractive } \\
\text { Index }\end{array}$ & $\begin{array}{c}\text { Central Bottle } \\
\text { Wavelength }(\boldsymbol{\mu m})\end{array}$ & $\begin{array}{c}\text { Upper Bottle } \\
\text { Wavelength }(\boldsymbol{\mu} \mathbf{m})\end{array}$ \\
\hline 1 & 0.788 & 0.799 \\
\hline 1.02 & 0.790 & 0.801 \\
\hline 1.05 & 0.792 & 0.803 \\
\hline 1.07 & 0.795 & 0.805 \\
\hline 1.09 & 0.797 & 0.808 \\
\hline 1.12 & 0.799 & 0.810 \\
\hline 1.14 & 0.802 & 0.813 \\
\hline 1.16 & 0.805 & 0.815 \\
\hline 1.18 & 0.807 & 0.818 \\
\hline 1.20 & 0.809 & 0.821 \\
\hline 1.23 & 0.812 & 0.822 \\
\hline
\end{tabular}

Table 7.2 The sensitivities calculated over each zone for the central and upper bottle regions.

\begin{tabular}{|c|c|c|}
\hline Refractive & Sensitivity Central & Sensitivity Upper \\
Index Region & Bottle $\left(\frac{n m}{R I U}\right)$ & Bottle $\left(\frac{n m}{R I U}\right)$ \\
\hline Gas & 86.9 & 87.8 \\
\hline Liquid & 123.6 & 121.8 \\
\hline Solid & 125.9 & 58.4 \\
\hline
\end{tabular}




\subsection{Gas Sensing}

The standard conditions for temperature and pressure (STP) and the gases' refractive indices (at low pressure) are close to air. The gases at very high pressure are also considered here and have led to refractive indices much higher than those at STP. The concentration of a gas depends on pressure and volume when the temperature is constant. The concentration of $\mathrm{CO}_{2}$ gas pressure concerning the refractive index is extracted from [98]. The central MBR's axial width is set at $W=1.5 \mu \mathrm{m}$ giving the structure shown in Figure 6.1. The geometry of each new structure is discretized with 50 grid points per micron to determine the series expansion space for the components of the relative permittivity tensor. The states are then computed using 50 Bessel terms and 89 axial terms for azimuthal mode order $20(q=20,1 \leq p \leq 50,-40 \leq n \leq+40)$. The $\mathrm{CO}_{2}$ gas at $40{ }^{\circ} \mathrm{C}$ is used from $300 \mathrm{psi}$ to $600 \mathrm{psi}$ (low pressure range). As the pressure increases, the refractive index of $\mathrm{CO}_{2}$ changes is summarized in Table 7.3. The wavelength and its shift due to refractive index change (pressure change) are tracked by plotting the states returned from each computation and identifying the lowest-order state profiles (axially directed) confined in the bottle (central region). The wavelength shift as a function of the ambient medium's refractive index (due to pressure) is plotted in Figure 7.2. This graph is used for computing sensitivity, i.e., change in wavelength $\Delta \lambda$ to change in refractive index $\Delta n$, over the entire refractive index range. The sensitivity for change in concentration of the gas at low pressure is determined to be $S=\frac{\Delta \lambda}{\Delta P} \Rightarrow S=\frac{\Delta \lambda}{\Delta n}=79.6 \frac{\mathrm{nm}}{R I U}$. 
Table 7.3 Wavelength and refractive index as a function of low pressure.

\begin{tabular}{|c|c|c|}
\hline Pressure (psi) & Refractive Index & Wavelength $(\boldsymbol{\mu m})$ \\
\hline 300 & 1.008 & 0.7889 \\
\hline 400 & 1.012 & 0.7891 \\
\hline 500 & 1.016 & 0.7895 \\
\hline 600 & 1.020 & 0.7898 \\
\hline
\end{tabular}

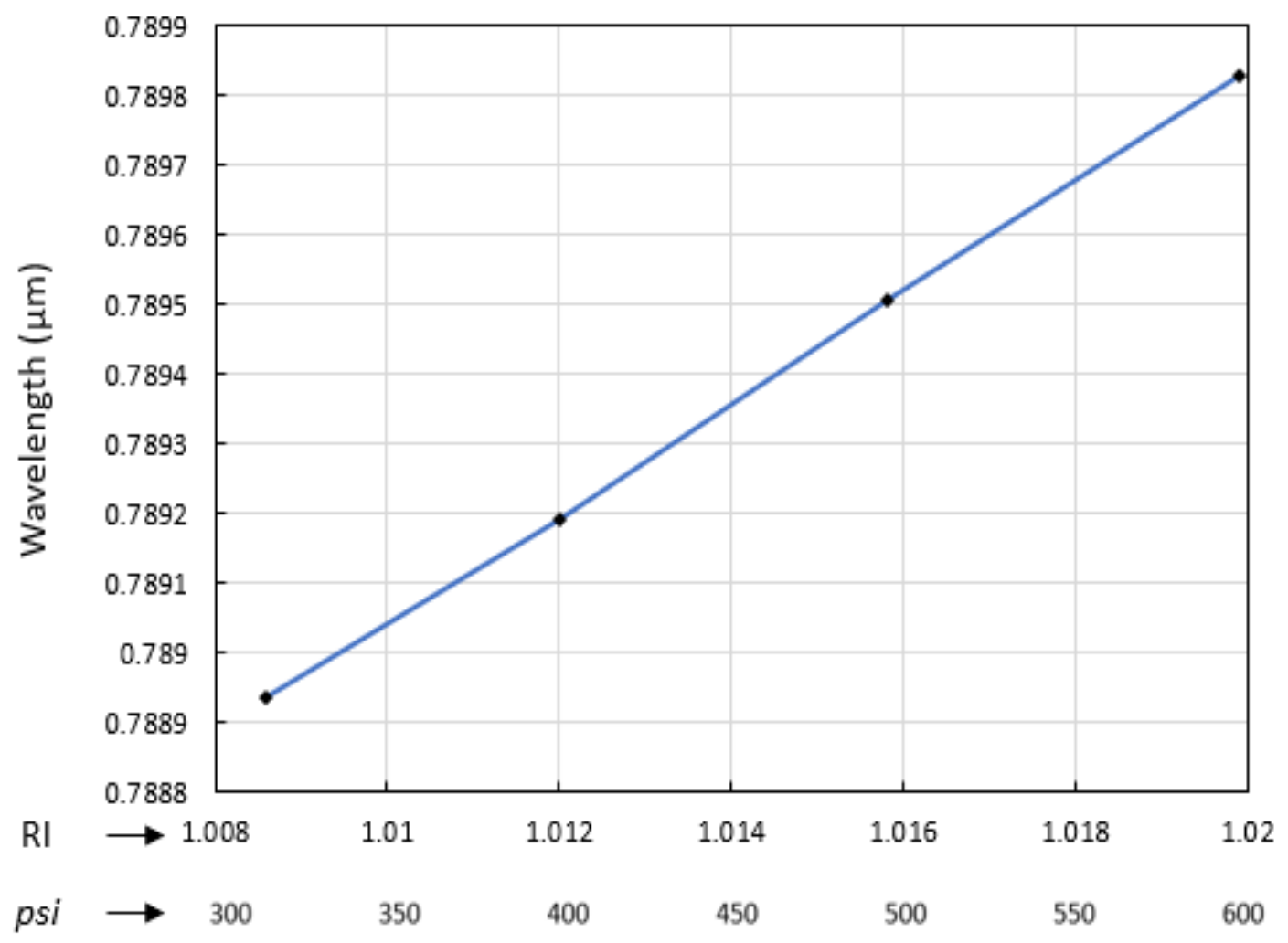

Figure 7.2 Changes in $\mathrm{CO}_{2}$ pressure (low range) versus wavelength shift. From the graph, the sensitivity of $79.6 \mathrm{~nm} / \mathrm{RIU}$ was calculated. 
The $\mathrm{CO}_{2}$ gas at $40{ }^{\circ} \mathrm{C}$ is used from 1100 psi to 1500 psi (high pressure range). As the pressure increases, the refractive index of $\mathrm{CO}_{2}$ changes are summarized in Table 7.4. The wavelength and its shift due to refractive index change are tracked by plotting the states returned from each computation and identifying the lowest-order state profiles (axially directed) confined in the bottle (central region). The wavelength shift as a function of the ambient medium's refractive index (due to high pressure) is plotted in Figure 7.3. This graph is used for computing sensitivity, i.e., change in wavelength $\Delta \lambda$ to change in refractive index $\Delta n$, over the entire refractive index range. The sensitivity for change in gas concentration was determined to be $S=\frac{\Delta \lambda}{\Delta P} \Rightarrow S=\frac{\Delta \lambda}{\Delta n}=118.2 \frac{\mathrm{nm}}{R I U}$.

Table 7.4 Wavelength and refractive index as a function of pressure in the high pressure domain.

\begin{tabular}{|c|c|c|}
\hline Pressure (psi) & Refractive Index & Wavelength $(\boldsymbol{\mu m})$ \\
\hline 1100 & 1.057 & 0.792 \\
\hline 1200 & 1.0805 & 0.795 \\
\hline 1250 & 1.1105 & 0.798 \\
\hline 1300 & 1.1333 & 0.801 \\
\hline 1400 & 1.149 & 0.803 \\
\hline 1500 & 1.158 & 0.804 \\
\hline
\end{tabular}




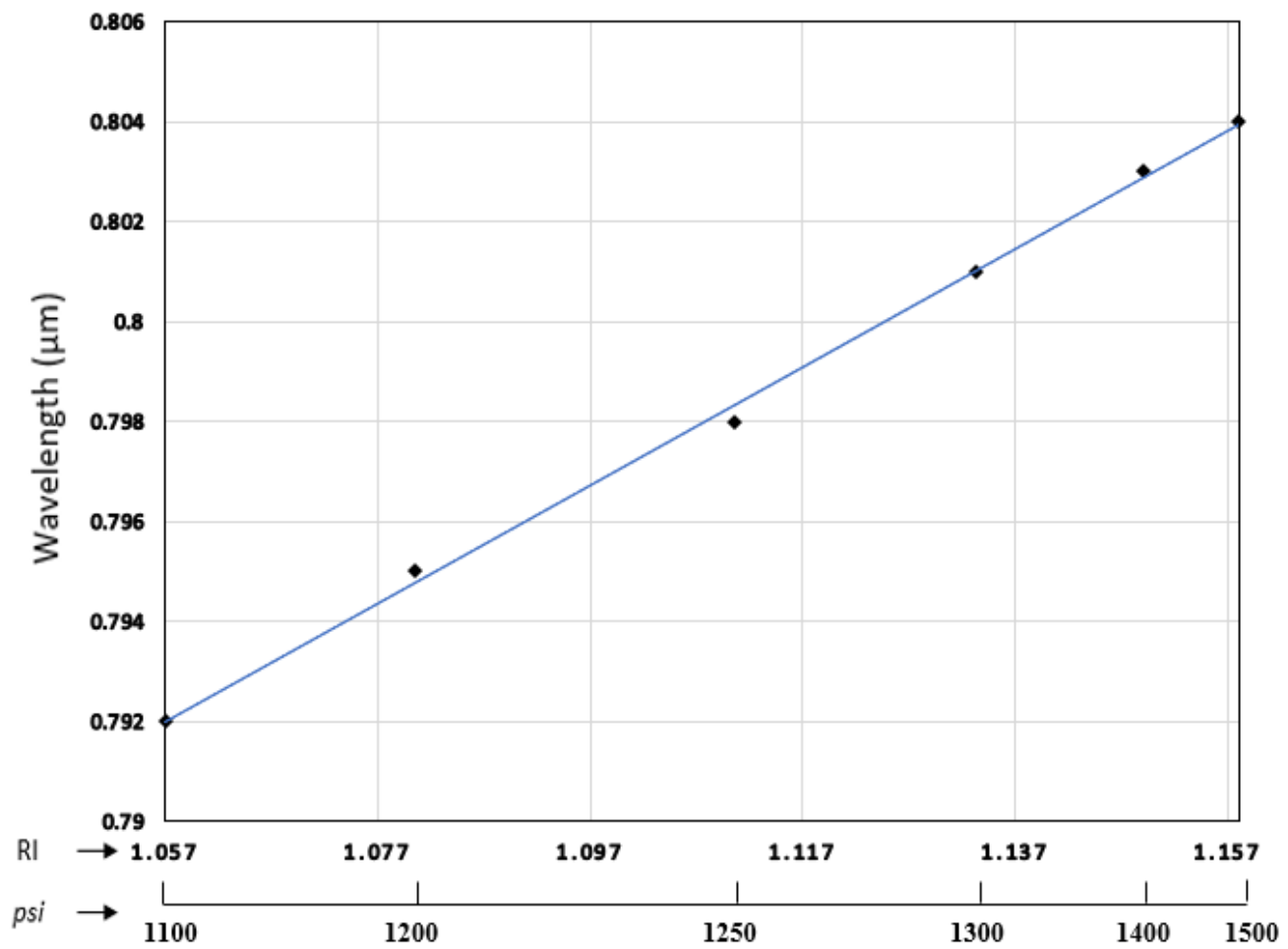

Figure 7.3 Changes in $\mathrm{CO}_{2}$ pressure (high range) versus the shift in wavelength.

Sensitivity was found to be $118.2 \mathrm{~nm} / \mathrm{RIU}$.

The gas/refractive index sensors discussed in Section 4.2.2 were built in hundreds of microns in dimension, and the refractive index sensitivity was $130 \frac{n m}{R I U}[60]$. The design presented in this thesis would allow the MBR to show $125.9 \frac{\mathrm{nm}}{\mathrm{RIU}}$ sensitivity in the solid region of the refractive index range. Hence, the refractive index sensor has demonstrated a comparable sensitivity. When $\mathrm{CO}_{2}$ is detected at a low pressure range using MBR, a sensitivity of $79.6 \frac{\mathrm{nm}}{R I U}$ was found, and at high pressure, $118 \frac{\mathrm{nm}}{R I U}$ was detected. It was observed that there is no significant change in wavelength trace; the result is expected to 
be low for the low pressure range since the refractive index of $\mathrm{CO}_{2}$ at STP is close to that of air at STP.

\subsection{Temperature Sensing}

The temperature sensor's essential features are the thermo-optic effect, $\beta$, and the linear thermal expansion, $\alpha$, as discussed in Section 4.2.1. The refractive index of the MBR composed of optical glass changes as the temperature varies. This property is called the thermo-optic effect expressed in (4.4). The change in refractive index, $\Delta n$, can be expressed as:

$$
\Delta n=\beta n_{0} \Delta T
$$

where $n_{0}$ is the initial refractive index, and $\Delta T$ is the temperature change. The MBR's temperature-dependent change in refractive index, $\Delta n$, was entered into the FFB mode solver as the temperature was varied from $10^{\circ} \mathrm{C}$ to $140{ }^{\circ} \mathrm{C}$ in the ambient medium. The geometry of each new structure is discretized with 50 grid points per micron to determine the series expansion space for the components of the relative permittivity tensor. The states are then computed using 50 Bessel terms and 89 axial terms for azimuthal mode order 20 $(q=20,1 \leq p \leq 50,-40 \leq n \leq+40)$. The lowest-order mode of the central bottle region in the axial direction is examined for azimuthal mode order 20 . The temperature concerning the refractive index change and its corresponding wavelength shift is summarized in Table 7.5. The wavelength and its shift due to the thermo-optic effect is traced by plotting the states returned from each computation in Figure 7.4. Note: The change in wavelength concerning the temperature change for MBR's material is extracted 
from the optical glass BK-7 datasheet, and approximate refractive index values were chosen for simulations [99].

Table 7.5 Refractive index as a function of temperature, which highlights the thermo-optic effect as a change in wavelength.

\begin{tabular}{|c|c|c|}
\hline Temperature $\left({ }^{\circ} \mathrm{C}\right)$ & Refractive Index & Wavelength $(\mu \mathrm{m})$ \\
\hline 10 & 1.4 & 0.7345 \\
\hline 20 & 1.5 & 0.7805 \\
\hline 30 & 1.6 & 0.8279 \\
\hline 40 & 1.7 & 0.8762 \\
\hline 50 & 1.8 & 0.9263 \\
\hline 60 & 1.9 & 0.9743 \\
\hline 70 & 2.0 & 1.0236 \\
\hline 80 & 2.1 & 1.0738 \\
\hline 90 & 2.2 & 1.1236 \\
\hline 100 & 2.3 & 1.1736 \\
\hline 110 & 2.4 & 1.2246 \\
\hline 120 & 2.5 & 1.2765 \\
\hline 130 & 2.6 & 1.3261 \\
\hline 140 & 2.7 & 1.3730 \\
\hline
\end{tabular}




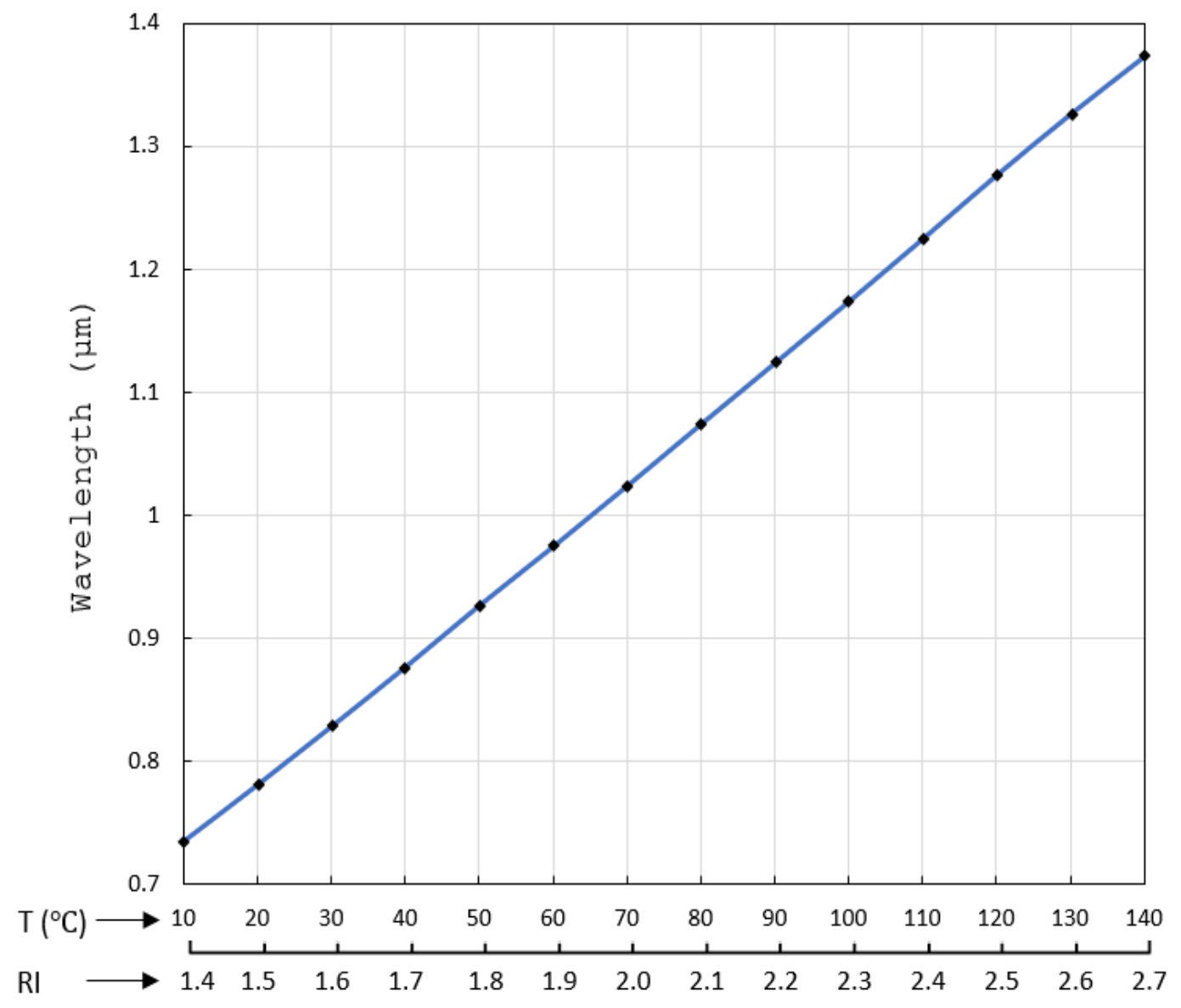

Figure 7.4 The solid line represents the thermo-optic effect, where the wavelength varies as a function of $10^{\circ} \mathrm{C}$ temperature changes at each point. The overall change in temperature will become $140^{\circ} \mathrm{C}$.

The MBR may also display a linear change in its size with a change in temperature due to thermal expansion (4.3). The coefficient of thermal expansion, $\alpha$, of optical glasses linearly increases from room temperature to a transformation range, where the glass becomes plastically deformable (when stress is sufficient to deform the shape permanently). Above this range, $\alpha$ rises again almost linearly but with a steeper slope until 
deformation occurs. The value of $\alpha=7.1 \times 10^{-6} \mathrm{~K}^{-1}$ is suitable from $-30{ }^{\circ} \mathrm{C}$ to $+70^{\circ} \mathrm{C}$. The examined structure will have the linear thermal expansion, and it will be applied to all its dimensions $(r, W, D$ and $T)$ defined in Figure 6.1. The length change can be expressed as:

$$
\Delta L=\alpha L_{0} \Delta T
$$

The linear thermal expansion will have a $0.001 \%$ impact on the MBR's dimensions and is expected to have a negligible effect on the resonator state wavelengths. The graph in Figure 7.4 is used for computing sensitivity, i.e., change in wavelength $\Delta \lambda$ to change in temperature $\Delta t$, over the entire temperature range. The temperature sensitivity of MBR was determined using $S=\frac{\Delta \lambda}{\Delta T}=4.9 \frac{\mathrm{nm}}{{ }^{\circ} \mathrm{C}}$.

Now that the temperature concerning effects on the MBR are determined. They are utilized for sensing the temperature effect of $\mathrm{CO}_{2}$ at a constant pressure of 600 psi and 1500 psi [98]. The air is replaced by $\mathrm{CO}_{2}$ in the ambient medium of the MBR. The central bottle region will detect the presence of the gas. The temperature variations from $40{ }^{\circ} \mathrm{C}$ to $120^{\circ} \mathrm{C}$ are selected because only this range of the refractive index was found for $600 \mathrm{psi}$ and $1500 \mathrm{psi}$. When the temperature change is applied, the refractive index of the $\mathrm{MBR}$ and the $\mathrm{CO}_{2}$ will both change. The geometry of each new structure is discretized with 50 grid points per micron to determine the series expansion space for the components of the relative permittivity tensor. The states are then computed using 50 Bessel terms and 89 axial terms for azimuthal mode order $20(q=20,1 \leq p \leq$ $50,-40 \leq n \leq+40)$. The wavelength and its shift are tracked by plotting the states returned from each computation and identifying the lowest-order state profiles (axially 
directed) confined in the bottle (central region). The wavelength as a function of the temperature change at 600 psi is plotted in Figure 7.5. This graph is used for computing sensitivity, i.e., change in wavelength $\Delta \lambda$ to change in temperature $\Delta T$, over the entire temperature range. All values related to 600 psi pressure are summarized in Table 7.6. The temperature sensitivity response of the $\mathrm{CO}_{2}$ is determined using $S=\frac{\Delta \lambda}{\Delta T}$. A sensitivity of approximately $4.9 \frac{\mathrm{nm}}{{ }^{\circ} \mathrm{C}}$ was detected. The wavelength as a function of the temperature change at 1500 psi is plotted in Figure 7.6 and summarized in Table 7.7. For computing sensitivity at high pressure changes in wavelength $\Delta \lambda$ to changes in temperature $\Delta T$, the entire temperature range was chosen and is detected to be $S=\frac{\Delta \lambda}{\Delta T}=4.8 \frac{\mathrm{nm}}{{ }^{\circ} \mathrm{C}}$.

The temperature-dependent sensitivity of MBR when air is in the ambient medium compared to when $\mathrm{CO}_{2}$ replaces air has no significant change in both the recorded sensitivities. The reason is that the refractive index of $\mathrm{CO}_{2}$ at STP (low pressure) is remarkably close to that of air at STP at low pressure. While at high pressure, the sensitivity is less than that achieved at low pressure. 


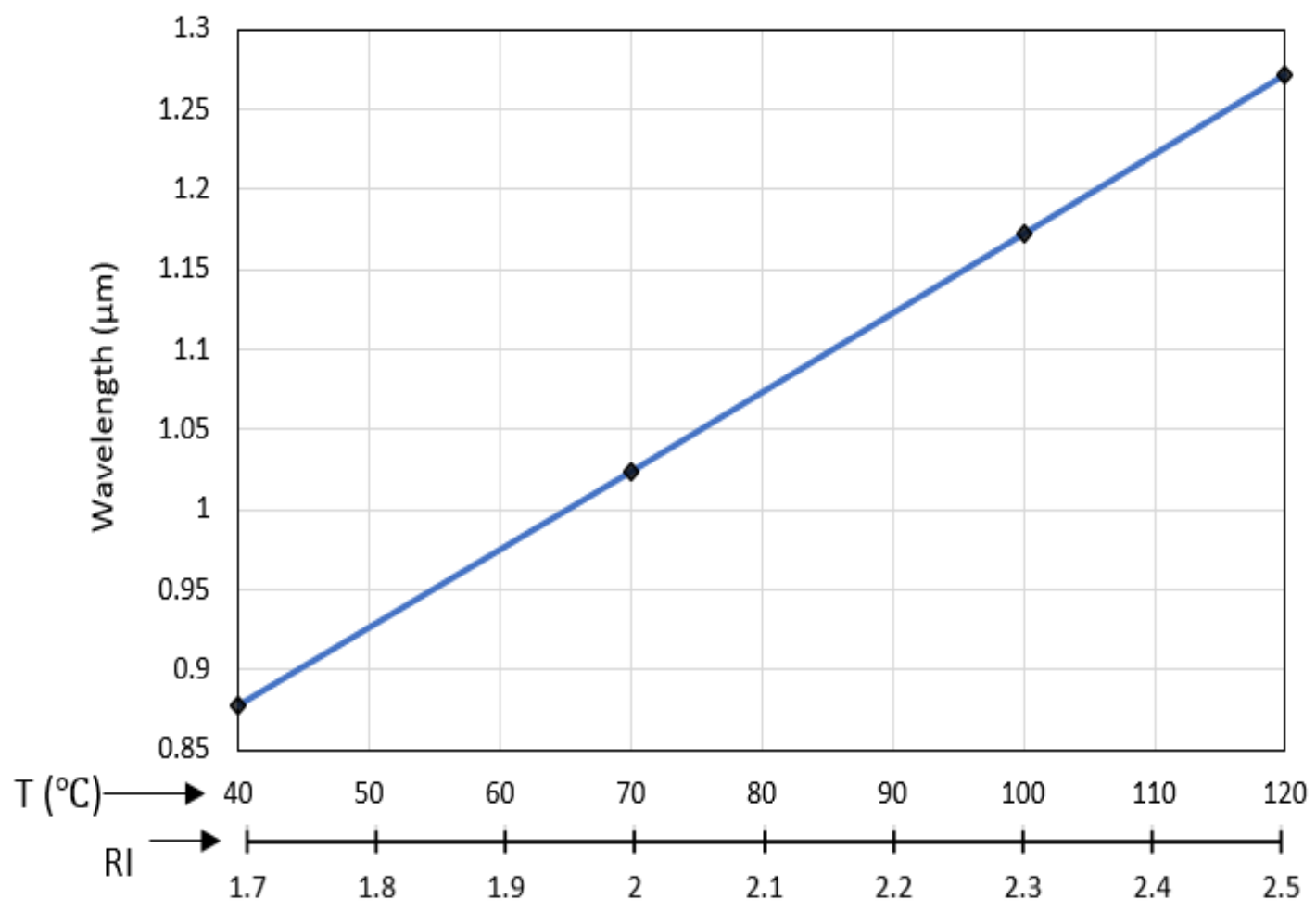

Figure 7.5 Changes in wavelength of $\mathrm{CO}_{2}$ gas at 600 psi with varying temperature. The sensitivity was determined to be $4.9 \mathrm{~nm} /{ }^{\circ} \mathrm{C}$.

Table 7.6 Refractive index as a function of temperature change for $\mathrm{MBR}$ and $\mathrm{CO}_{2}$ gas (at 600 psi) causes the wavelength to shift.

\begin{tabular}{|c|c|c|c|}
\hline $\begin{array}{c}\text { Temperature } \\
\left({ }^{\circ} \mathrm{C}\right)\end{array}$ & $\begin{array}{c}\text { Refractive Index } \\
\text { MBR }\end{array}$ & $\begin{array}{c}\text { Refractive Index } \\
\mathbf{C O}_{2}\end{array}$ & $\begin{array}{c}\text { Wavelength } \\
(\boldsymbol{\mu} \mathbf{m})\end{array}$ \\
\hline 40 & 1.7 & 1.019 & 0.8772 \\
\hline 70 & 2 & 1.016 & 1.0242 \\
\hline 100 & 2.3 & 1.014 & 1.1727 \\
\hline 120 & 2.5 & 1.013 & 1.2719 \\
\hline
\end{tabular}




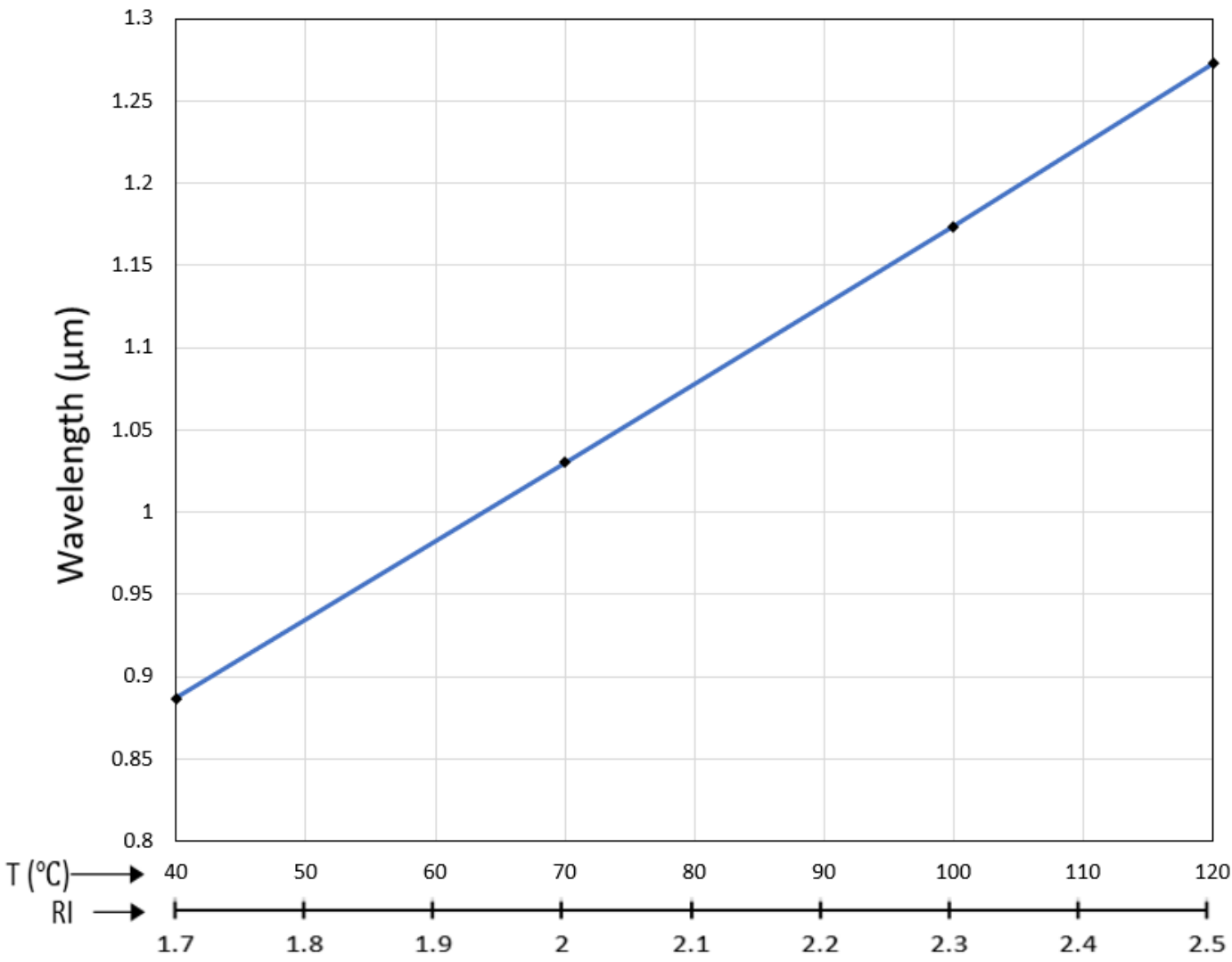

Figure $7.6 \mathrm{CO}_{2}$ gas at 1500 psi with varying temperature versus wavelength gives $4.8 \mathrm{~nm} /{ }^{\circ} \mathrm{C}$ sensitivity.

Table 7.7 Refractive index as a function of temperature change for the $\mathrm{MBR}$ and $\mathrm{CO}_{2}$ gas at 1500 psi causes the wavelength to shift.

\begin{tabular}{|c|c|c|c|}
\hline $\begin{array}{c}\text { Temperature } \\
\left({ }^{\circ} \mathbf{C}\right)\end{array}$ & $\begin{array}{c}\text { Refractive } \\
\text { Index MBR }\end{array}$ & $\begin{array}{c}\text { Refractive } \\
\text { Index CO} \mathbf{C}_{\mathbf{2}}\end{array}$ & $\begin{array}{c}\text { Wavelength } \\
(\boldsymbol{\mu m})\end{array}$ \\
\hline 40 & 1.7 & 1.158 & 0.8866 \\
\hline 70 & 2 & 1.059 & 1.0301 \\
\hline 100 & 2.3 & 1.043 & 1.1736 \\
\hline 120 & 2.5 & 1.039 & 1.2728 \\
\hline
\end{tabular}


For better performance of the sensor, high sensitivity is required. In the literature review, mainly temperature sensors have recorded sensitivities in $p m /{ }^{\circ} \mathrm{C}$. For example, one of the temperature sensors, discussed in Section 4.2.1, using MBR built to hundreds of microns in dimensions, has recorded sensitivity in $1.3 \mathrm{pm} /{ }^{\circ} \mathrm{C}$ [68], whereas the temperature sensor designed in this thesis has a sensitivity of $4.9 \mathrm{~nm} /{ }^{\circ} \mathrm{C}$. Sensitivity in $\mathrm{nm} /{ }^{\circ} \mathrm{C}$ is larger than $\mathrm{pm} /{ }^{\circ} \mathrm{C}$. Hence, the demonstrated sensor is a better option for temperature sensor detection.

\subsection{Chemical Sensing}

The HMBR geometry was chosen for chemical sensor simulations. As discussed in Section 6.2, the MBR is hollowed-out, creating HMBR for two reasons. One reason is to allow chemicals to flow through the HMBR's inner (hollow-core) region. The second reason is to have thin walls for optical mode confinement. The narrower the walls, the more likely the mode will leak out in the surrounding. The mode's evanescent fields will interact with the chemical found in the surrounding medium. As the hollowed region's refractive index changes, the mode's wavelength will shift. Here, the lowest-order mode (axially directed) confined in the thin walls of the HBMR (central bottle region) is examined.

Each new structure is discretized with 50 grid points per micron to determine the series expansion space for the components of the relative permittivity tensor. The states are then computed using 50 Bessel terms and 89 axial terms for azimuthal mode order 20 ( $q=$ $20,1 \leq p \leq 50,-40 \leq n \leq+40)$. The index of refraction was varied from air to a chemical vapor of $n=1.6$ in increments of 0.05 . This range includes the refractive index 
of the HMBR's material (BK-7). The mode evolution for the lowest-order mode in the axial direction is displayed in Figure 7.7. When the range of refractive index increases and gets closer to the HMBR's refractive index, the intensity of optical mode confinement in the thin wall also increases from $n=1.4$ to $n=1.6$. The wavelength and its shift are tracked by plotting the states returned from each computation and identifying the lowestorder state profiles (axially directed) confined in the HMBR (central inner region). The wavelength as a function of the inner region's refractive index is plotted in Figure 7.8. All values related to this figure are summarized in Table 7.8. The graph gradually increases until it reaches close to the optical glass's refractive index (1.5168), where a linearly increasing behavior from 1.5 to 1.6 of refractive index was observed. This graph is used for computing sensitivity, i.e., change in wavelength $\Delta \lambda$ to change in refractive index $\Delta n$, over the entire range of the refractive index. In Figure 7.9, as the refractive index increases, the sensitivity at each point varies. 


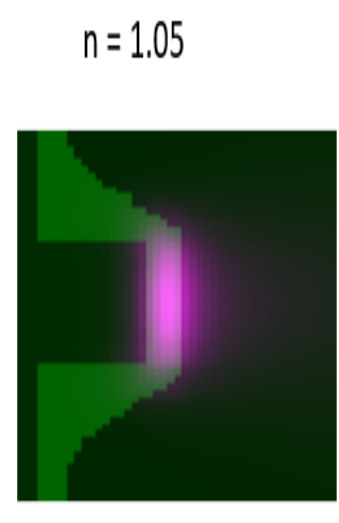

$$
n=1.1
$$

$n=1.15$

$n=1.2$
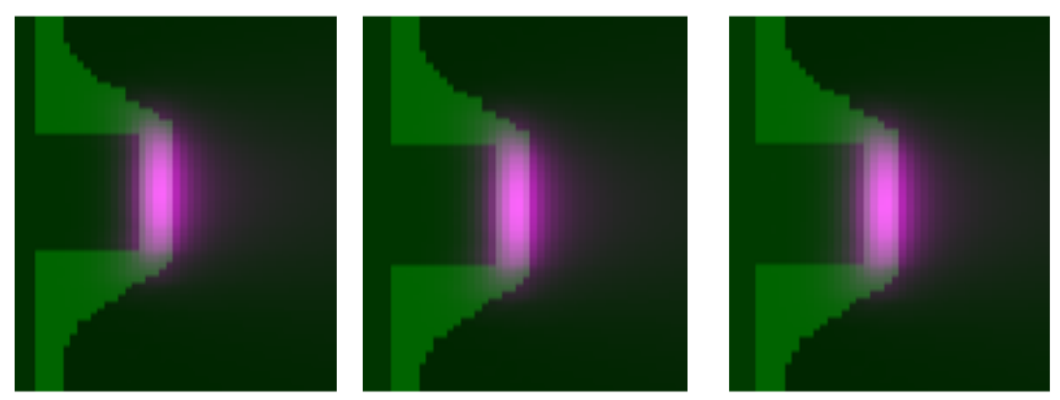

$n=1.25$

$n=1.3$

$n=1.35$

$n=1.4$
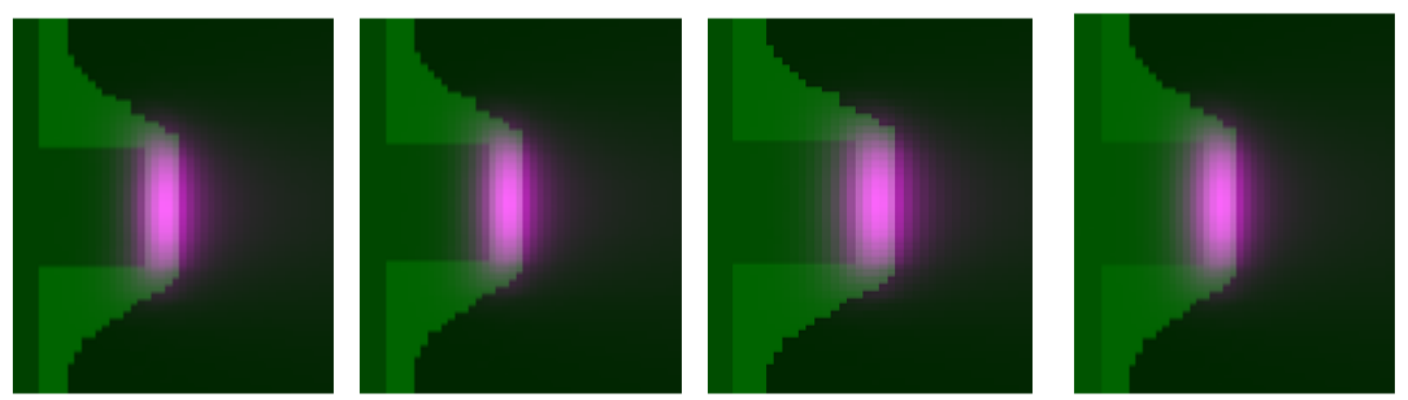

$$
n=1.45
$$

$$
n=1.5
$$

$n=1.55$

$n=1.6$
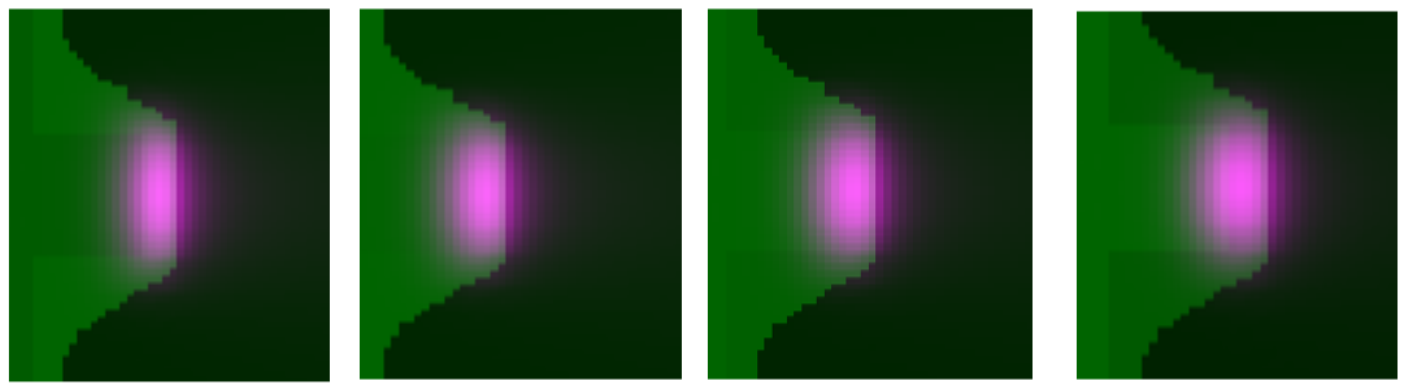

Figure 7.7 The refractive index (n) variation is visualized in the central bottle. When the $n$ value of the hollow notch gets close enough to that of the optical glass at $n=1.5$ and $n=1.55$, an increase in the intensity of the confinement optical mode is observed. 


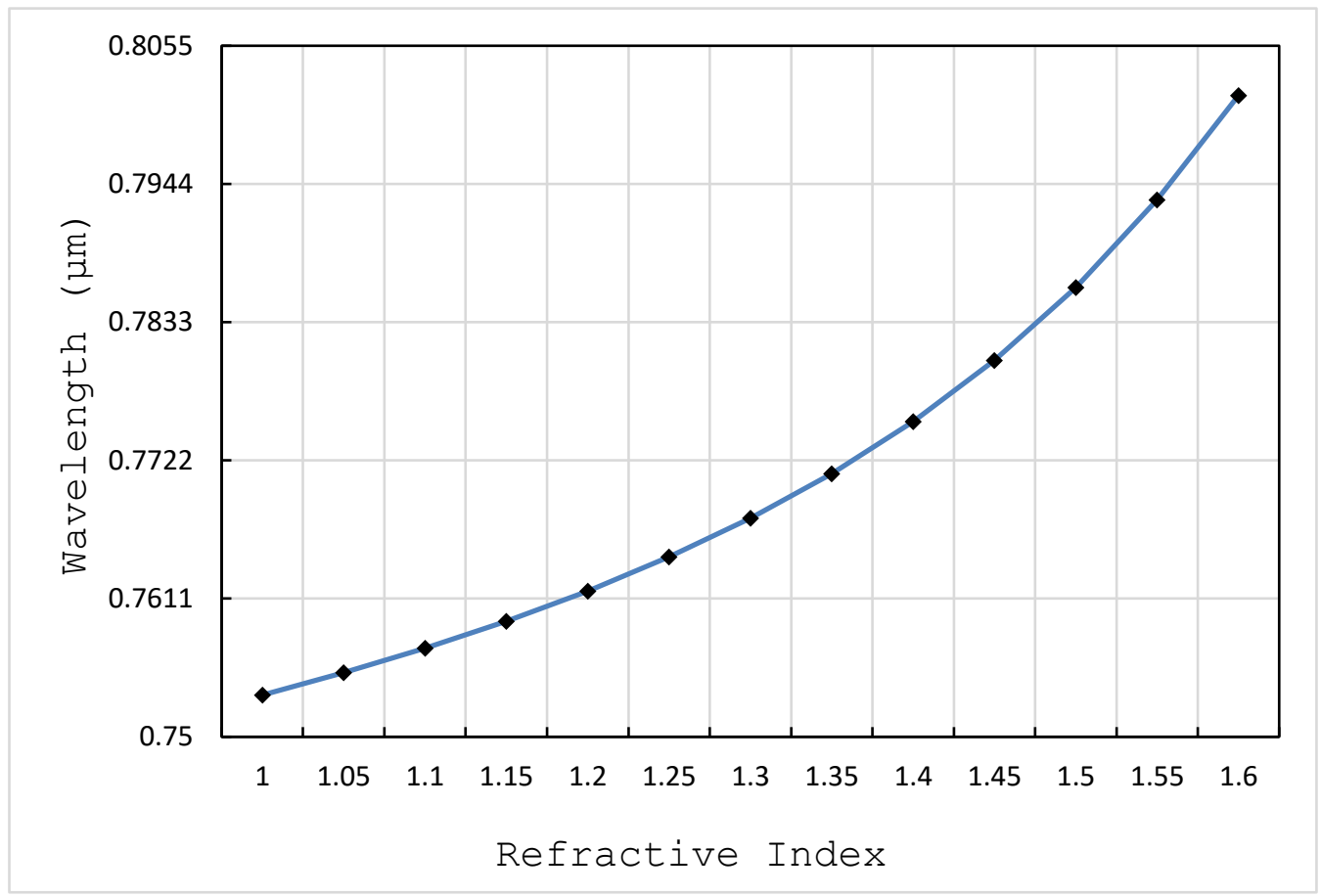

Figure 7.8 Bottle confined resonator state wavelength response as a function of the internal hollow region's refractive index change (lowest order mode axially directed).

Table 7.8 Wavelength shift as a function of refractive index.

\begin{tabular}{|c|c|}
\hline Refractive Index & Wavelength $(\boldsymbol{\mu m})$ \\
\hline 1.00 & 0.7533 \\
\hline 1.05 & 0.7551 \\
\hline 1.10 & 0.7571 \\
\hline 1.15 & 0.7592 \\
\hline 1.20 & 0.7617 \\
\hline 1.25 & 0.7644 \\
\hline 1.30 & 0.7675 \\
\hline 1.35 & 0.7711 \\
\hline 1.40 & 0.7753 \\
\hline 1.45 & 0.7802 \\
\hline 1.50 & 0.7860 \\
\hline 1.55 & 0.7931 \\
\hline 1.60 & 0.8015 \\
\hline
\end{tabular}




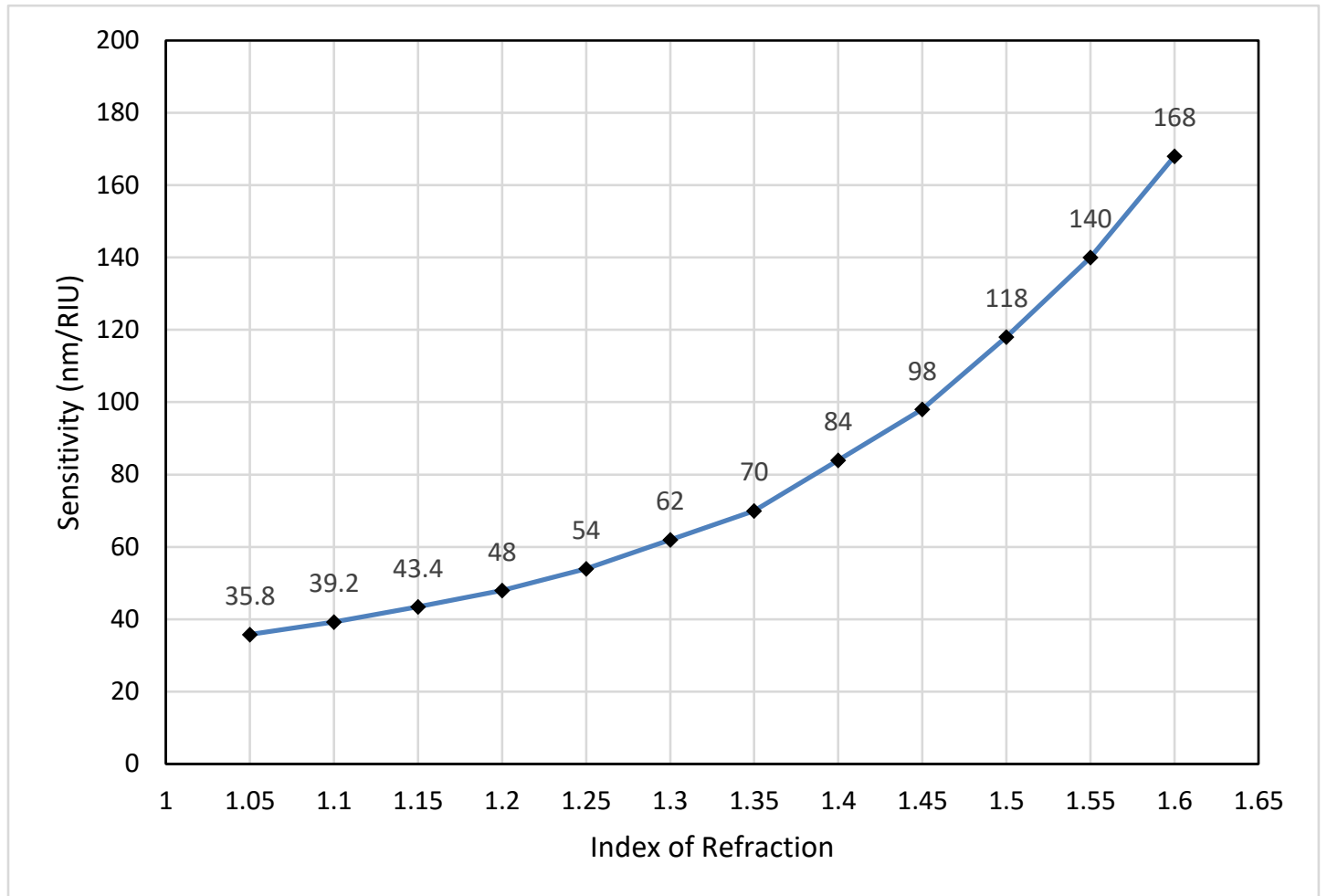

Figure 7.9 The sensitivity of a chemical sensor for a generalized range concerning the next point is identified.

A concentrated single compound chemical whose refractive index varies with changes in molar concentrated volume was chosen for further simulations. Liquid benzene was selected, and concentration changes from $89.31 \mathrm{~cm}^{3} \cdot \mathrm{mol}^{-1}$ to $91.13 \mathrm{~cm}^{3} \cdot \mathrm{mol}^{-1}$ were found in [100]. As the concentration changes, the related refractive index will vary, causing a shift in wavelength, as summarized in Table 7.9. The geometry of each new structure is discretized with 50 grid points per micron to determine the series expansion space for the components of the relative permittivity tensor. The states are then computed using 50 Bessel terms and 89 axial terms for azimuthal mode order 20 ( $q=20,1 \leq p \leq$ 
$50,-40 \leq n \leq+40)$. The wavelength and its shifts are tracked by plotting the states returned from each computation. The lowest-order state profiles (axially directed) confined in the thin wall of the HMBR (central inner region) are identified and examined. The wavelength as a function of the inner region's refractive index (related to benzene's concentration) is plotted in Figure 7.10. It is observed that as the concentration increases, the resonance wavelength decreases. This graph is used for computing sensitivity, i.e., change in wavelength $\Delta \lambda$ to change in refractive index $\Delta n$, over the entire range of the refractive index. The sensitivity detected is $S=\frac{\Delta \lambda}{\Delta \text { concentration }} \Rightarrow S=\frac{\Delta \lambda}{\Delta n}=123.8 \frac{\mathrm{nm}}{\mathrm{RIU}}$.

Table 7.9 Refractive index as a function of the concentration of liquid benzene.

\begin{tabular}{|c|c|c|}
\hline $\begin{array}{c}\text { Concentration } \\
\left(\mathbf{c m}^{\mathbf{3}} \cdot \mathbf{m o l}^{\mathbf{- 1}} \mathbf{)}\right.\end{array}$ & $\begin{array}{c}\text { Refractive } \\
\text { Index }\end{array}$ & $\begin{array}{c}\text { Wavelength } \\
(\boldsymbol{\mu} \mathbf{m})\end{array}$ \\
\hline 89.32 & 1.4984 & 0.7858 \\
\hline 89.94 & 1.4941 & 0.7853 \\
\hline 90.55 & 1.4898 & 0.7848 \\
\hline 91.17 & 1.4855 & 0.7842 \\
\hline
\end{tabular}




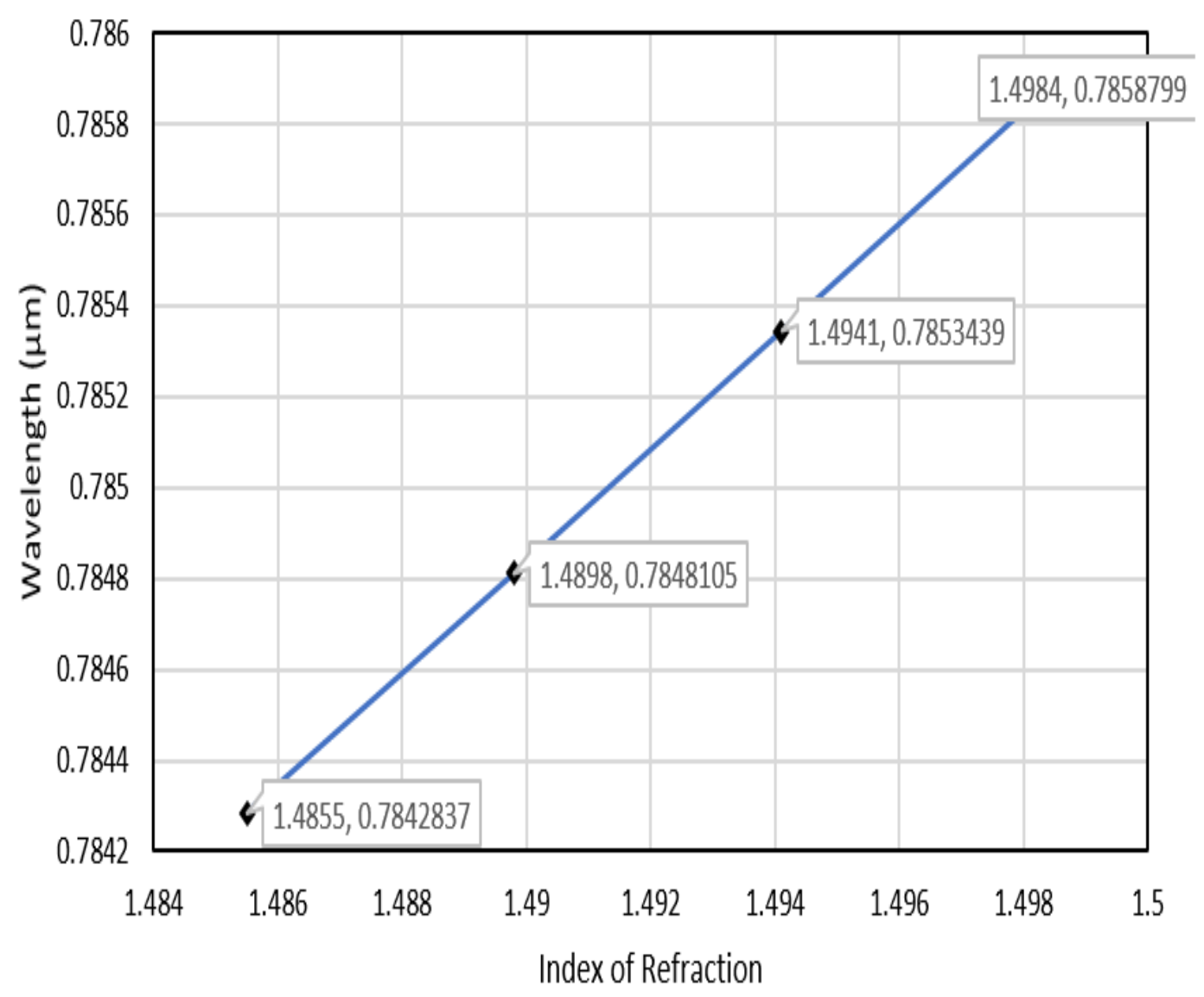

Figure 7.10 As the concentration of benzene changes, the refractive index changes causing a shift in the wavelength, and a sensitivity of $123.8 \mathrm{~nm} / R I U$ was achieved.

Similarly, a mixed ratio of two compounds is chosen, and their response is observed. Chlorobenzene's relative permittivity (2.3253) is very close to that of HMBR (2.300). As chlorobenzene's concentration increases, its refractive index moves closer to the HMBR and reaches a cutoff wavelength. According to the definition, the cutoff wavelength is the minimum wavelength where the fiber supports a single-mode, and below it, higher-order modes are allowed to propagate through the fiber. Hence, the HMBR can 
no longer support the lowest-order axially directed mode allowing the first-order mode (axially directed) to propagate. As the chlorobenzene's concentration changes, the related refractive index vary and is summarized in Table 7.10. It is observed from the table that with an increase in chlorobenzene's concentration from $101.7 \mathrm{~cm}^{3} . \mathrm{mol}^{-1}$ to $103.2 \mathrm{~cm}^{3} . \mathrm{mol}^{-1}$, the resonance wavelength decreases [100]. The geometry of each new structure is discretized with 50 grid points per micron to determine the series expansion space for the components of the relative permittivity tensor. The states are then computed using 50 Bessel terms and 89 axial terms for azimuthal mode order 20 $(q=20,1 \leq p \leq 50,-40 \leq n \leq+40)$. The wavelength and its shift are tracked by plotting the states returned from each computation. The first-order state profiles (axially directed) confined in the thin wall of the HMBR (central inner region) are identified and examined. The wavelength as a function of the inner region's refractive index (related to chlorobenzene's concentration) is plotted in Figure 7.11. This graph is used for computing sensitivity, i.e., change in wavelength $\Delta \lambda$ to change in refractive index $\Delta n$, over the entire range of the refractive index. The sensitivity is calculated to be $S=\frac{\Delta \lambda}{\Delta \text { concentration }} \Rightarrow \frac{\Delta \lambda}{\Delta n}=$ $185.9 \frac{n m}{R I U}$

Table 7.10 Refractive index and wavelength as a function of chlorobenzene concentration.

\begin{tabular}{|c|c|c|}
\hline Concentration $\left(\mathbf{c m}^{\mathbf{3}} \cdot \mathbf{m o l}^{\mathbf{- 1}}\right)$ & Refractive Index & Wavelength $(\boldsymbol{\mu m})$ \\
\hline 101.75 & 1.5249 & 0.7727 \\
\hline 102.23 & 1.5239 & 0.7725 \\
\hline 102.75 & 1.5229 & 0.7723 \\
\hline 103.26 & 1.5219 & 0.7721 \\
\hline
\end{tabular}




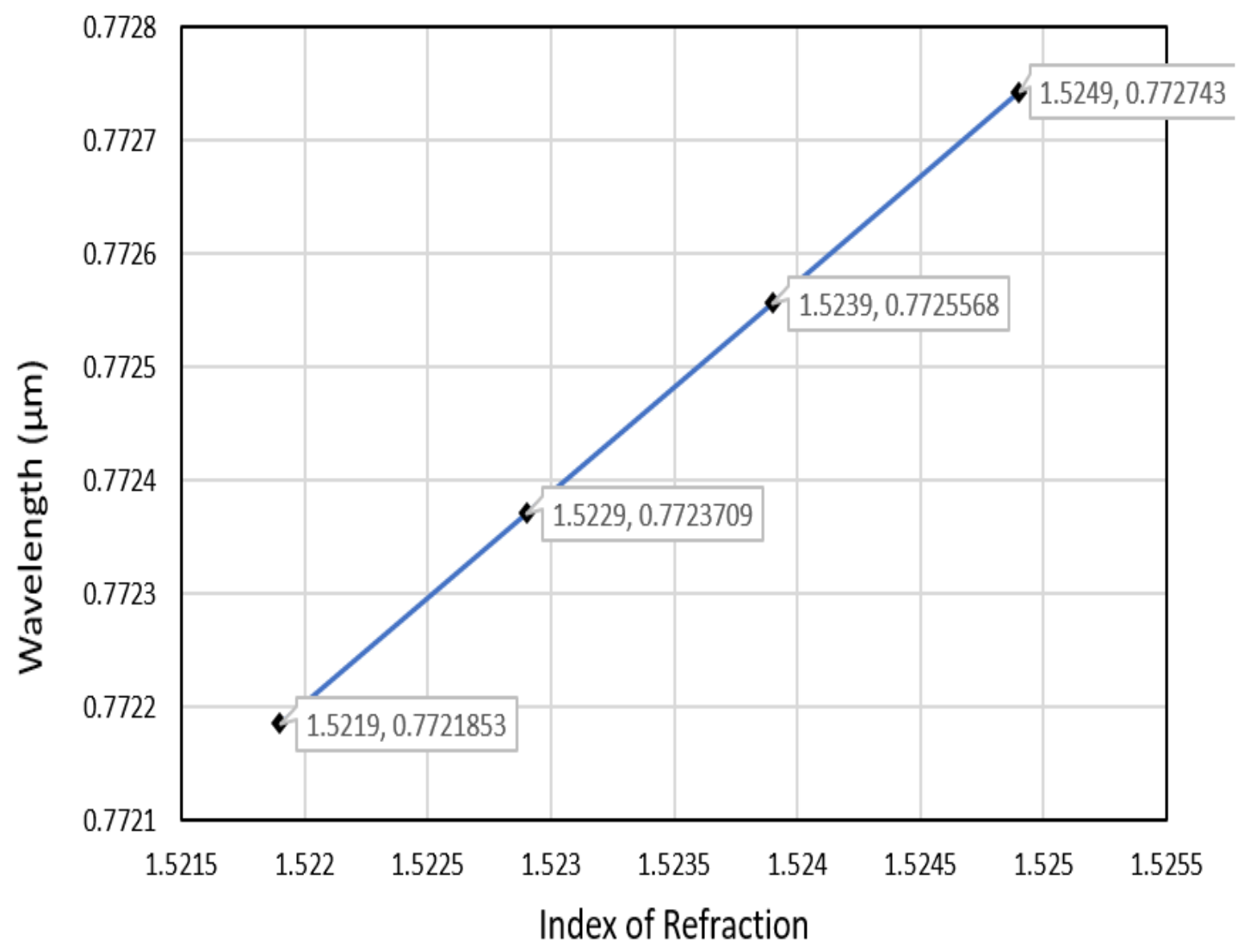

Figure 7.11 The concentration of liquid chlorobenzene with the first-order mode wavelength change gives a $185.9 \mathrm{~nm} / \mathrm{RIU}$ sensitivity.

As discussed in Sections 3.4 and 6.2, it is expected from the HMBR to be more sensitive when an internal (hollow) and external (ambient) medium's refractive index vary simultaneously. This can be analogous to dipping the sensor in the analyte. The following test simulations involve the inner-outer regions of the HMBR - the refractive index changes from 1 to 1.2 with an increment of 0.025 . The geometry of each new structure is discretized with 50 grid points per micron to determine the series expansion space for the components of the relative permittivity tensor. The states are then computed using 50 
Bessel terms and 89 axial terms for azimuthal mode order $20(q=20,1 \leq p \leq 50,-40 \leq$ $n \leq+40)$. The wavelength and its shift are tracked by plotting the states returned from each computation. The lowest-order state profiles (axially directed) confined in the thin wall of the HMBR (central inner-outer region) are identified and examined. The refractive index change and the corresponding change in resonance wavelength for the lowest-order mode in the axial direction are summarized in Table 7.11. The confined mode's evolution as the refractive index changes is displayed in Figure 7.12. It is observed that thin walls allow the mode to leak outside, and its evanescent fields will interact with both inner-outer regions. The wavelength as a function of the inner region's and outer region's refractive index change is plotted in Figure 7.13. This graph is used for computing sensitivity, i.e., change in wavelength $\Delta \lambda$ to change in refractive index $\Delta n$, over the entire range of the refractive index. A sensitivity of $S=\frac{\Delta \lambda}{\Delta n}=176.5 \frac{\mathrm{nm}}{R I U}$ was calculated.

Table 7.11 Wavelength change as a function of refractive index in the inner-outer regions of $H M B R$.

\begin{tabular}{|c|c|}
\hline Refractive Index & Wavelength $(\boldsymbol{\mu m})$ \\
\hline 1.000 & 0.7533 \\
\hline 1.025 & 0.7572 \\
\hline 1.050 & 0.7614 \\
\hline 1.075 & 0.7657 \\
\hline 1.100 & 0.7701 \\
\hline 1.125 & 0.7746 \\
\hline 1.150 & 0.7792 \\
\hline 1.174 & 0.7838 \\
\hline 1.200 & 0.7884 \\
\hline
\end{tabular}



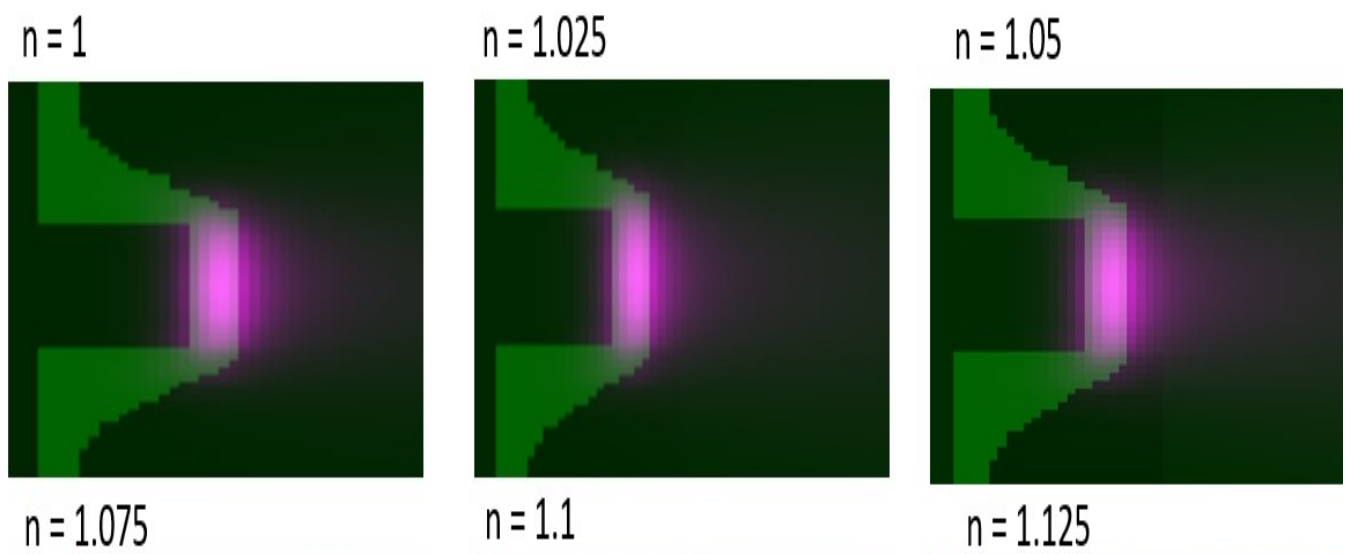

$$
n=1.1
$$

$$
n=1.125
$$
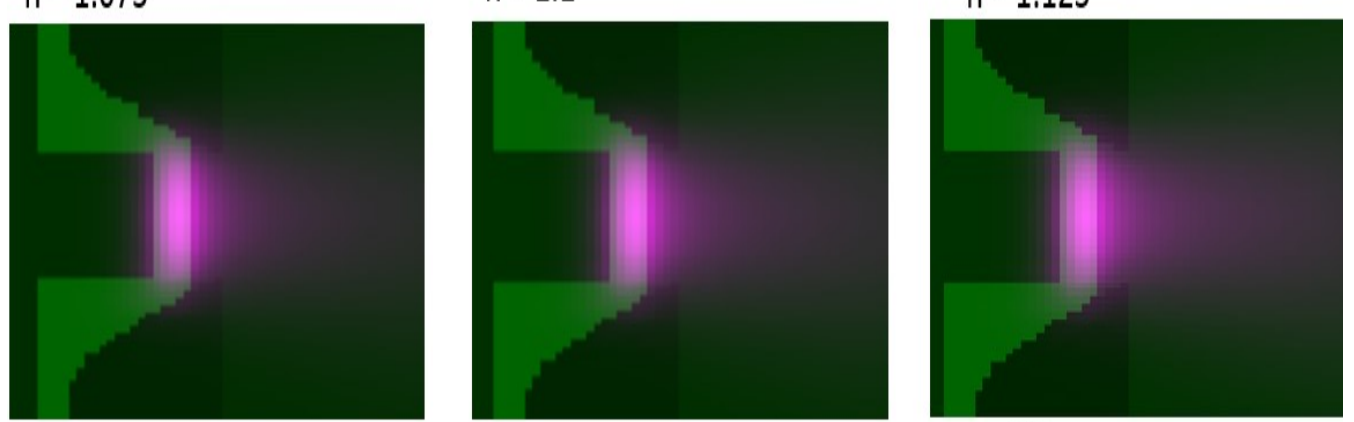

$n=1.15$

$$
n=1.175
$$

$$
n=1.2
$$
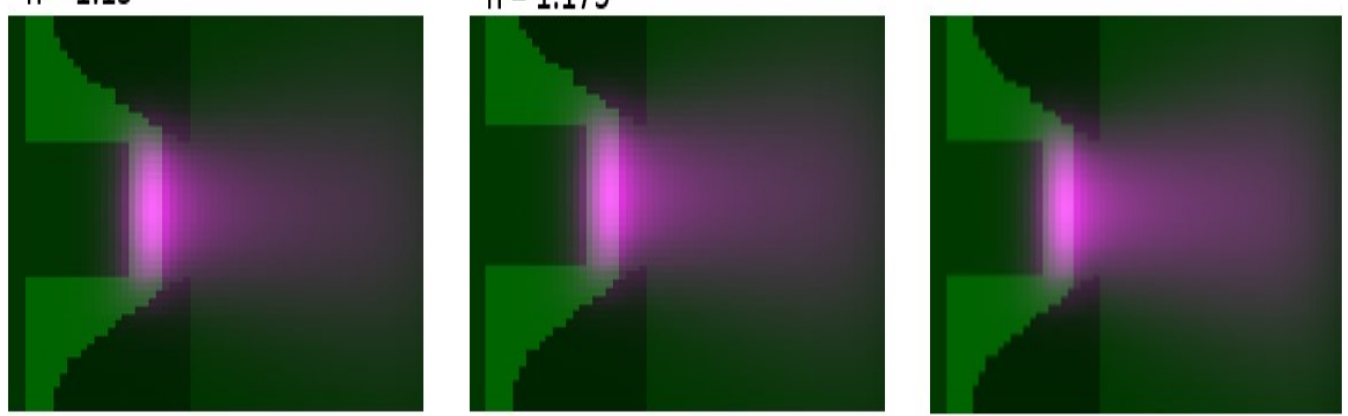

Figure 7.12 The lowest-order mode (axially directed) confined in the thin wall of the HMBR. Its evanescent fields interact with the inner and outer medium's refractive index. 


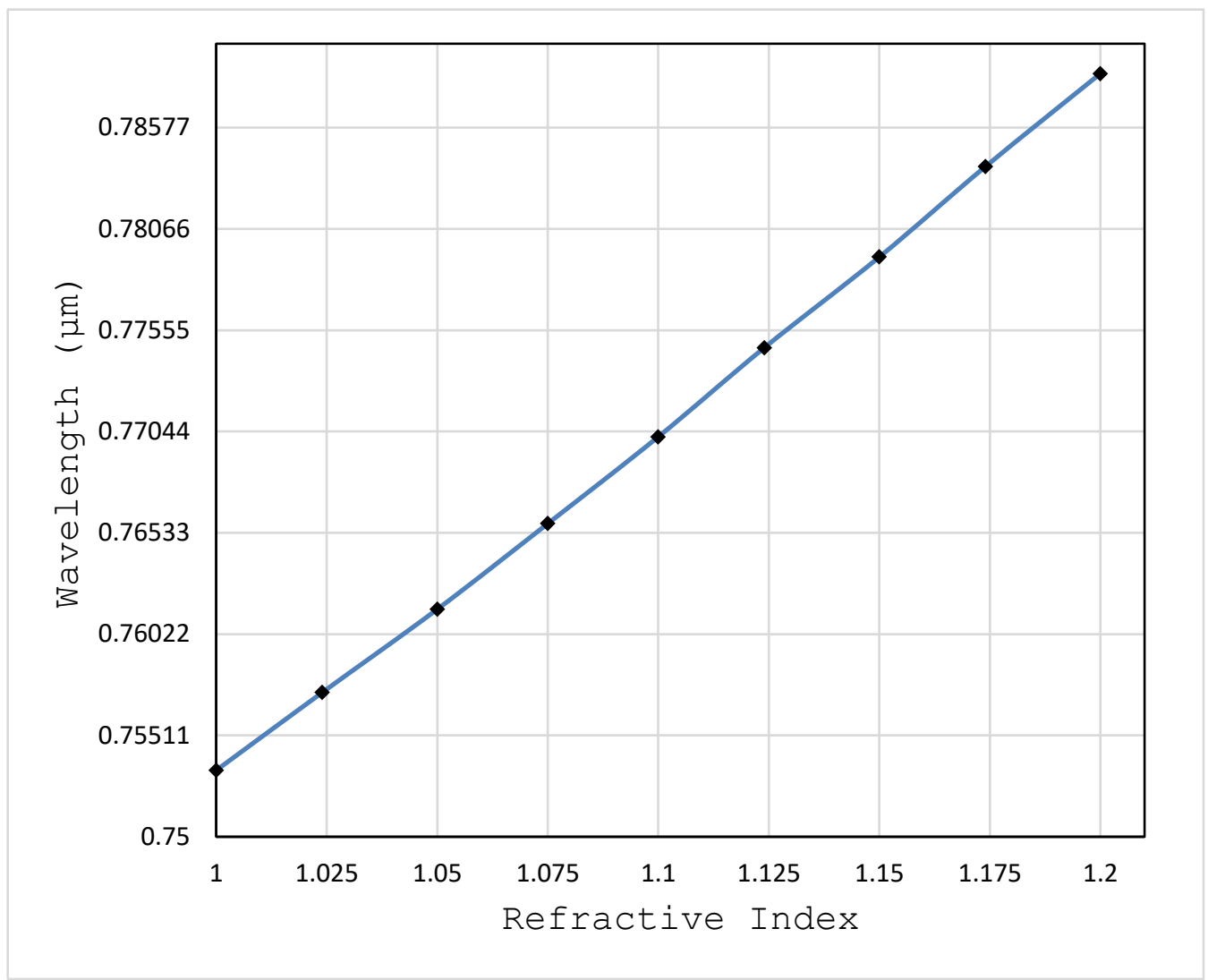

Figure 7.13 Bottle confined resonator state wavelength response as a function of the internal and external region's refractive index change for the lowest order mode in the axial direction. A sensitivity of $176.5 \mathrm{~nm} / \mathrm{RIU}$ was detected.

There were two chemical sensors built and discussed in this section. One of them uses an inner (hollow) region for refractive index variations, see Figure 7.8. The other uses inner and outer regions at the same time for refractive index variations, see Figure 7.13. It is monitored at $n=1.5$ of the inner region refractive index, and the wavelength is $\lambda=$ $0.786 \mu \mathrm{m}$, and at $n=1.2$ of the inner-outer region, the wavelength is $\lambda=0.788 \mu \mathrm{m}$. These wavelengths are comparable. A new chart is generated with the refractive index 
ranging from 1 to 1.2 with an increment of 0.05 with corresponding wavelength shift (for both the regions) for a proper comparison; see Table 7.12. This table's values are used for further sensitivity calculations, i.e., change in wavelength $\Delta \lambda$ to change in refractive index $\Delta n$, over the entire range of the refractive index for both regions; see Figure 7.14. Since the refractive index is the same for both regions, the sensitivity becomes directly proportional to the wavelength; the higher the wavelength, the more sensitive that sensor will be. The inner region has a sensitivity of $S=\frac{\Delta \lambda}{\Delta n}=41.7 \frac{\mathrm{nm}}{\mathrm{RIU}}$, and the inner-outer region refractive index change has a sensitivity of $S=\frac{\Delta \lambda}{\Delta n}=176.1 \frac{n m}{R I U}$. This indicates the innerouter regions' refractive index variation increases the sensitivity by $\sim 24 \%$.

Table 7.12 For comparison, the refractive index change and wavelength variations related to the inner-outer region and inner region.

\begin{tabular}{|c|c|c|}
\hline Refractive Index & Inner-Outer region $(\boldsymbol{\lambda}-\boldsymbol{\mu} \mathbf{m})$ & Inner region $(\boldsymbol{\lambda}-\boldsymbol{\mu} \mathbf{m})$ \\
\hline 1 & 0.7533 & 0.7533 \\
\hline 1.05 & 0.7614 & 0.7551 \\
\hline 1.1 & 0.7701 & 0.7571 \\
\hline 1.15 & 0.7792 & 0.7592 \\
\hline 1.2 & 0.7884 & 0.7617 \\
\hline
\end{tabular}




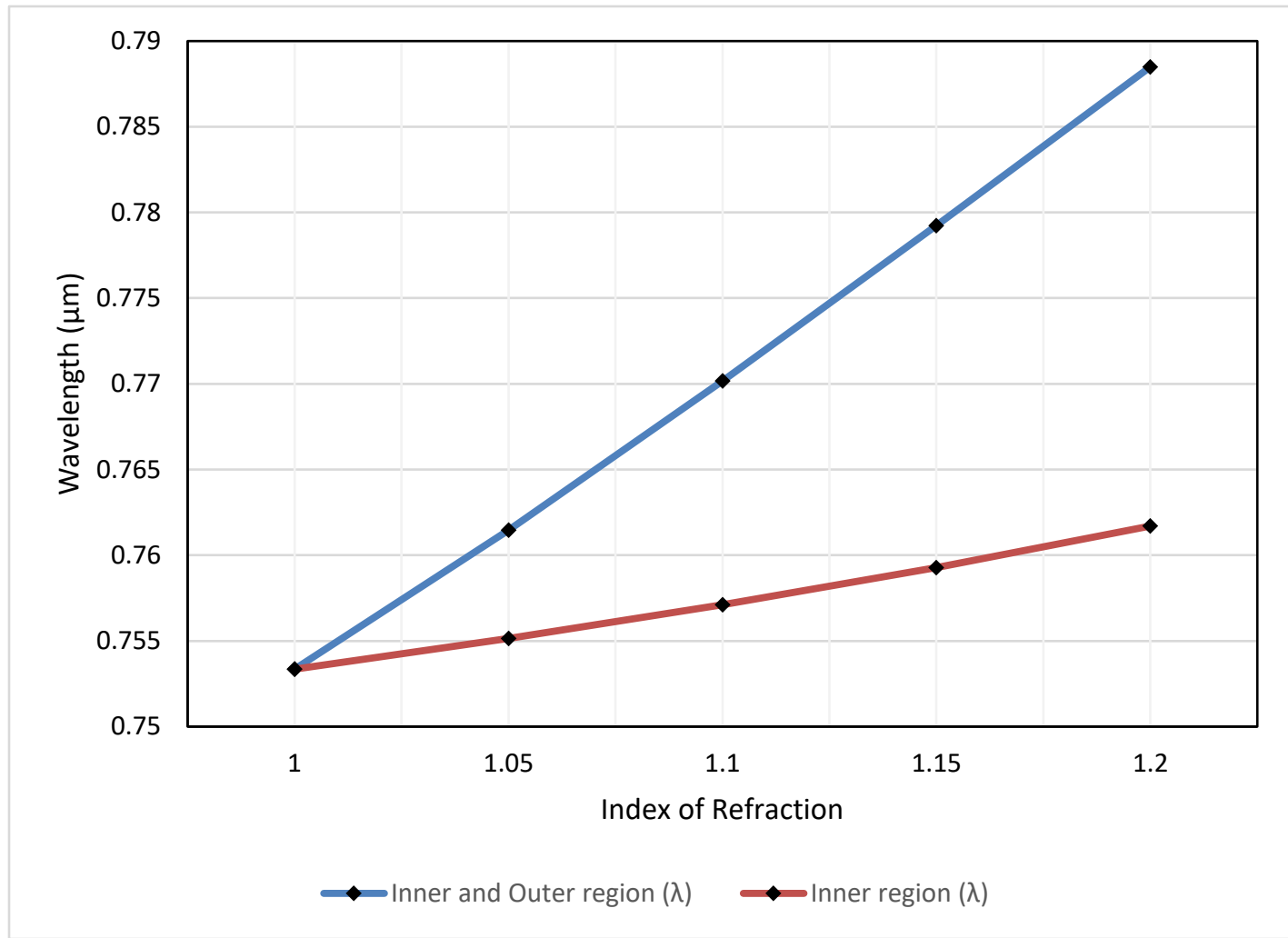

Figure 7.14 The refractive index changes from $n=1$ to 1.2, changes the wavelengths (inner and inner-outer regions) for the lowest-order mode axially directed.

Presently, chemical sensors with wall thickness as low as $10 \mu m$ have a sensitivity of $33 \frac{n m}{R I U}$ [75]. In this thesis, the designed sensor has a wall thickness of $0.25 \mu m$, which gives a $123.8 \mathrm{~nm} / \mathrm{RIU}$ and $185.9 \frac{\mathrm{nm}}{\mathrm{RIU}}$ sensitivity for liquid benzene and chlorobenzene, respectively. When only the inner region's refractive index ( $n=1$ to 1.5 ) is varied, a sensitivity up to $168 \frac{\mathrm{nm}}{R I U}$ is sensed. When both internal and external surrounding mediums' refractive index ( $n=1$ to 1.2 ) are varied at the same time, a sensitivity of $176.5 \frac{\mathrm{nm}}{R I U}$ is detected. 
Theoretical results were obtained from the designed solid, and hollow-core MBR configured as optical sensors. The MBR structures' modal property was examined as a function of the ambient medium's refractive index and temperature change. $\mathrm{A} \mathrm{CO}_{2}$ gas at a fixed temperature was used in gas sensing. Then, $\mathrm{CO}_{2}$ at a fixed pressure was used for temperature sensing. In the last section, the HMBR was configured for detecting chemical variations in the internal (hollow-core) and external (ambient) regions. It was also used for chemical concentration detection, i.e., benzene and its compound chlorobenzene, flowing through the hollow-core.

The next chapter sums up the theoretical findings and presents ideas for future work. 


\section{Chapter 8: Conclusions and Future Work}

A detailed overview of some of the fundamental concepts related to micro resonators was discussed, including WGMs, their cavity structures, characteristic properties, and various sensing mechanisms with related examples and applications. MBR was chosen to investigate sensors using spectral decomposition software from all available micro resonators, because it is the least explored resonator. The FFB mode solver requires the resolution of the electric and magnetic medium properties, and only the first derivatives of the field components give this approach faster convergence than using the wave equations directly.

As a sensor, the optical micro solid-core bottle resonator has shown great potential when built up to $1.5 \mu \mathrm{m}$. When placed under different sensing situations, such as bulk refractive index sensing, gas sensing (high pressure) and temperature sensing, it has demonstrated approximate sensitivities of $125.9 \frac{\mathrm{nm}}{\mathrm{RIU}}, 118.2 \frac{\mathrm{nm}}{\mathrm{RIU}}$, and $4.9 \frac{\mathrm{nm}}{{ }^{\circ} \mathrm{C}}$, respectively. The hollow-core bottle resonator with walls thinned to $0.25 \mu \mathrm{m}$ only with the internal sensing feature activated for a generalized refractive index range gives a sensitivity estimation of $168 \frac{\mathrm{nm}}{\mathrm{RI}}$. For liquid benzene and chlorobenzene, sensitivities of $123.8 \frac{\mathrm{nm}}{\mathrm{RIU}}$ and 185.9 $\frac{n m}{R I U}$ were achieved, respectively. When both the internal and external sensing environments were activated, the sensitivity was improved to $176.5 \frac{\mathrm{nm}}{\mathrm{RIU}}$. Most of the sensors demonstrated in this thesis are better than those commonly encountered in the literature review using similar resonator approaches. 
In the future, this design could be a better candidate for a strain-stress sensor or a magnetic field sensor. It could also be implemented in other applications such as a delay line filter, laser resonator, or nonlinear computations. The fabrication and testing process of the designed MBR should also be continued in the future. 


\section{Appendices}

\section{Appendix A}

\section{A.1 Fabrication of a solid MBR}

Efforts were placed into creating an MBR within tens of micron size, which corresponds to the theoretical work. Primarily, MBR fabrication involves softeningcompression techniques and heated fiber pulling techniques, but numerous attempts with different methods were used in this thesis. These techniques use a laser writing machine (SF-100) and UV light Aligner for MBR designs(exposing the pattern) on the fiber. The initial experiments were performed on a quartz wafer, instead of optical fiber, because they are easier to handle. Once the optimized waveguides were achieved on the quartz wafer, they could be transferred easily on fibers with slight variations in the photolithographic process.

The MBR was fabricated by creating two ring indentations around the fiber, and the distance between the rings will be the central bottle region. The intent is to fabricate the complete structure as small as possible using SF-100. During experiments with the quartz wafer and fiber, around $6 \mu \mathrm{m}$ and 4-5 $\mu \mathrm{m}$ were achieved. Note, this mask-less laser writing machine has a limitation of $4.5 \mu \mathrm{m}$.

The objective was to create a ten micron-sized MBR using limited resources available at our lab facility. The first few experiments involved a direct-writing machine, i.e., SF-100. The transferrable image was created for SF-100 using MS Paint. Each pixel of the image would correspond to a few microns in width in the fabrication process. Once 
the optimized width was achieved for ring indentations, the axial distance between rings can be adjusted the same way. This axial width is our region of interest, the optical MBR, as discussed in Section 6.1. The final mask or image is then adjusted for optimized results.

The SF-100 we used functionally limited in that the automated stage for placing the fiber directly under the UV light was malfunctioning. Due to this limited functionality, manual placement was done, which was time-consuming and inaccurate. Due to the inaccuracy of the placement, the UV light was reflected, and sometimes duplicate images were observed on the fiber, resulting in blurry images, which did not correspond to the original design.

\section{A.2 Quartz Wafer}

The entire photolithographic process was undertaken to start with cutting and scribing the quartz wafer sample. This was done to ensure the sample fit the spinner and the aligner and then scribed so that any changes during the photolithographic process could be effectively documented. The next step is to piranha clean the quartz wafer. The piranha solution comprises a 3:1 mixture of concentrated sulfuric acid $\left(\mathrm{H}_{2} \mathrm{SO}_{4}\right)$ with hydrogen peroxide $\left(\mathrm{H}_{2} \mathrm{O}_{2}\right)$. This solution is dangerous when hot, and the reaction in acidic piranha is self-starting. The samples were placed in solution and then heated to 100 ${ }^{\circ} \mathrm{C}$ and cooled once they reached the required temperature. The quartz wafer was removed, and the remaining acid solution was washed off. The glass was dehydrated for 20 minutes in the oven, and the temperature was set to $180^{\circ} \mathrm{C}$ to remove excess moisture. The SU-8-2 layer was deposited and placed in the spinner for even coating by ramping it up from 0 to 
$1000 \mathrm{rpm}$ for 5 seconds to obtain the ring indentations' desired thickness. The sample was prebaked for $1 \mathrm{~min}$ at $65{ }^{\circ} \mathrm{C}$ and soft-baked for another $3 \mathrm{~min}$ at $95{ }^{\circ} \mathrm{C}$. SF-100 micromachining was used to expose the UV light on the sample. The length of the exposure period was calculated by consulting the SU-8-2 datasheet to determine the amount of energy needed to harden the photoresist. Then, the energy amount was divided by the amount of energy the aligner lamp was able to produce per second. After exposure, post exposure baking (PEB) was completed at $65^{\circ} \mathrm{C}$ and $95^{\circ} \mathrm{C}$ for 1 minute each. The pattern created on the sample was developed using a SU-8-2 developer. This photolithography procedure's last step was to hard-bake by placing the sample on the hotplate for $200{ }^{\circ} \mathrm{C}$ for 1 minute. For optimized results, this process was repeated at various exposure times with different stage-level settings. The best result achieved on the quartz wafer is shown in Figure A.2.1. When repeated on optical fiber, the same procedure gives waveguides of a minimum of $4 \mu \mathrm{m}$, as shown in Figure A.3.1.

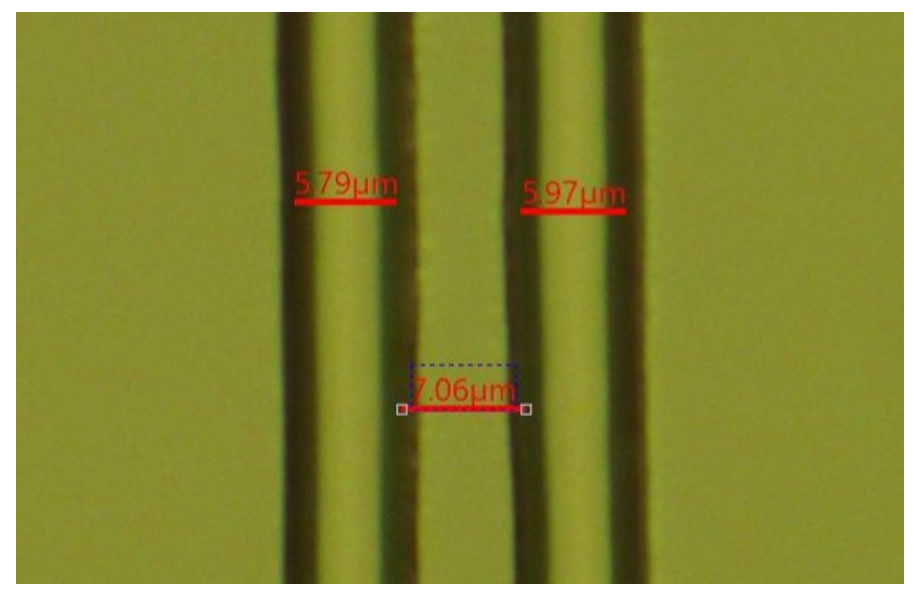

Figure A.2.1 At an exposure time of 6.5 seconds with a stage level set to 575, around 6micron ring indentations were achieved with a bottle region of 7 microns. 


\section{A.3 Fiber}

The optimized photolithographic process determined from the quartz wafer was repeated on fiber optics. The 2-D waveguides will be converted into 3-D waveguides. To do this, there were challenges, such as how to evenly deposit the SU-8-2 since fiber cannot be spun in the spinner as it might break into several pieces and fly out of the spinner. The deposition can be done by hand painting, dipping, or spraying. Hand painting is not the best option because the results are blurry waveguides, and it is a tedious task, see Figure A.3.1. Before and after, the negative photoresist deposition process will follow the same procedure as mentioned in Section A.2. However, the baking time will vary and is summarized in Table A.3.1.
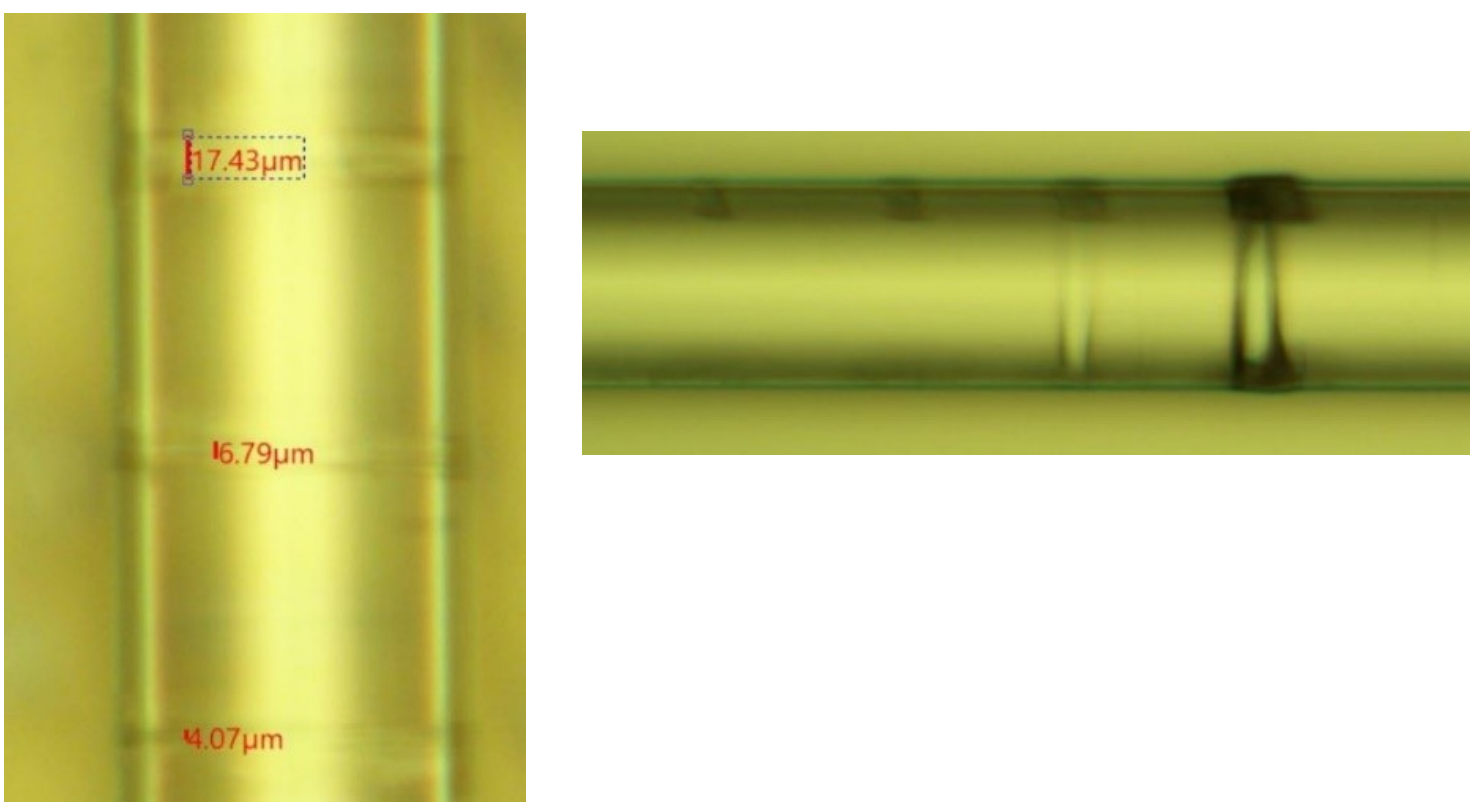

Figure A.3.1 The left is the front view where ring indentations (minimum $4 \mu \mathrm{m}$ ) are created and the right is the back view of the fiber. 
On the first dip coating of SU-8-2, a perfectly smooth-shaped bottle structure was formed, but it did not give the desired size because they were hundreds of microns in size (refer to Figure A.3.2). The dipped fiber went through all the relevant procedures, as discussed in Section A2, and gave approximately $30 \mu \mathrm{m}$ long rings, as shown in Figure A.3.3. The pre-and post-baking times are mentioned in Table A.3.1.

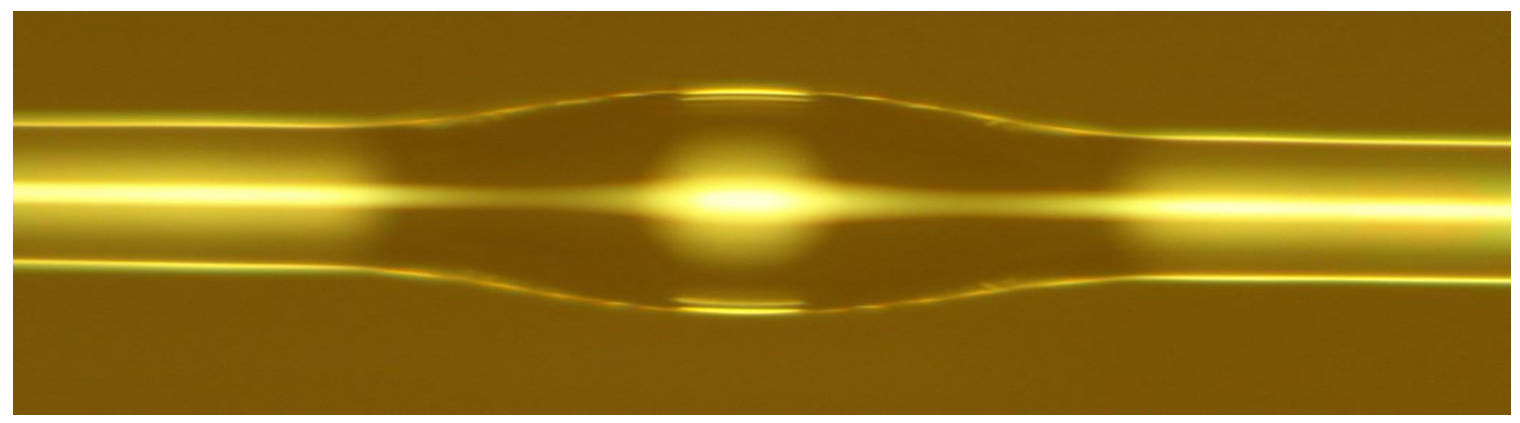

Figure A.3.2 Lab fabricated solid micro-optic bottle resonator using SU-8 (negative resist) dipcoated twice.

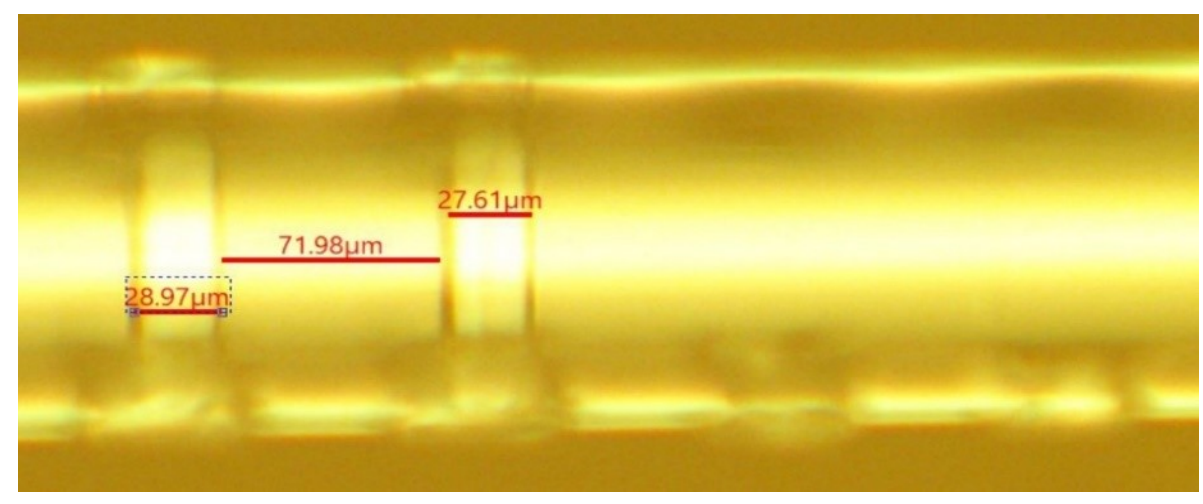

Figure A.3.3 The dimension achieved on a dipped coated fiber after going through the photolithographic process was $\sim 30 \mu \mathrm{m}$ wide. 
The thick waveguides obtained from the dip coating can be improved by spraying a thin layer of SU-8-2 on the fiber, as shown in Figure A.3.4. Before and after, the negative photoresist deposition process will follow the same procedure as mentioned in Section A.2. The result is a blurry waveguide, and as predicted, the dimensions were reduced by half of the size produced from dip coating. The pre-and post-baking times are summarized in Table A.3.1. The waveguides obtained from sprayed coating could be improved by depositing the heated SU-8-2 layer. Heating SU-8-2 creates a thinner consistency and results in reduced dimensions of the waveguide.

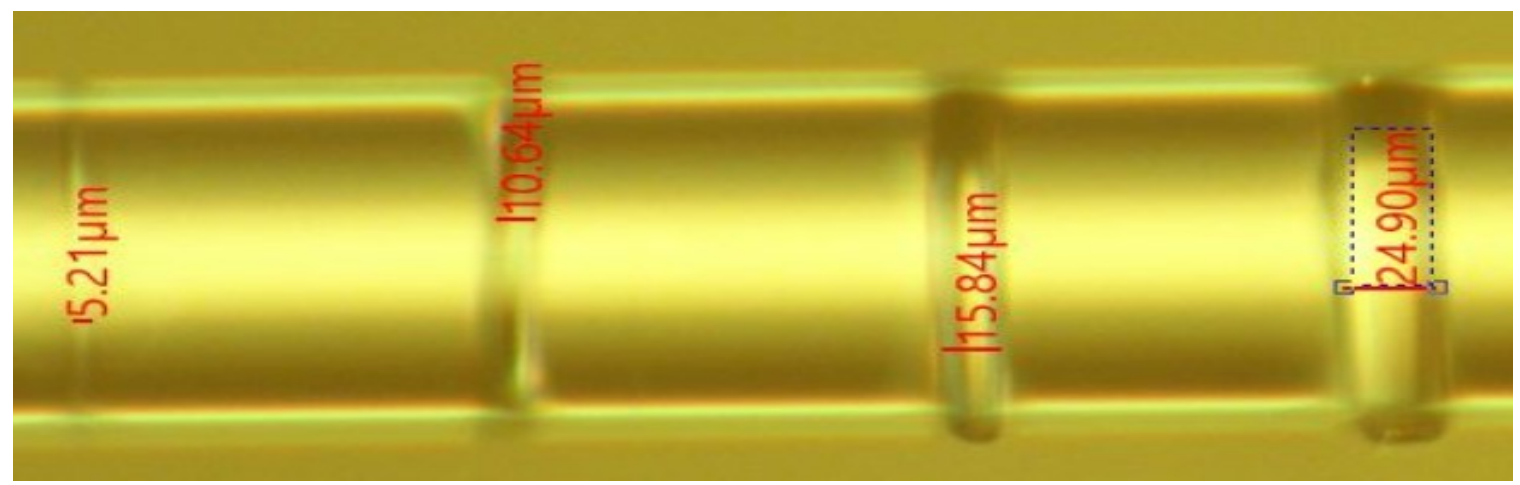

Figure A.3.4 Another attempt was made to create different size ring indentations with a varying width of the bottle resonator. Sprayed SU-8 while rotating the fiber 17 seconds exposed by laser writing machine, SF-100.

In the following procedure, the SU-8-2 was heated in the ultrasonic heater with a lid-on to maintain the temperature at $60^{\circ} \mathrm{C}$. The air spray holder was warmed up to $60^{\circ} \mathrm{C}$, and the heated SU-8-2 was poured into it, so the temperature would not drop. Once the photoresist is sprayed on the fiber, the solution rested for 10 minutes to settle the airborne particles. Before and after, the negative photoresist deposition process will follow the same 
procedure as mentioned in Section A.2. It was exposed to the UV-Aligner for 30 seconds instead of the SF-100. The waveguide achieved shows good results, but it was unable to reduce the size, see Figure A.3.5. The pre-and post-baking times are summarized in Table A.3.1.The final step was to hard-bake the sample for 2 minutes at $200{ }^{\circ} \mathrm{C}$, which was ramped up from room temperature to the peak temperature, then wait 2 minutes and start ramping down until it reached room temperature again. Exposure through the Aligner has given good results around $30 \mu m$, see Figure A.3.6.

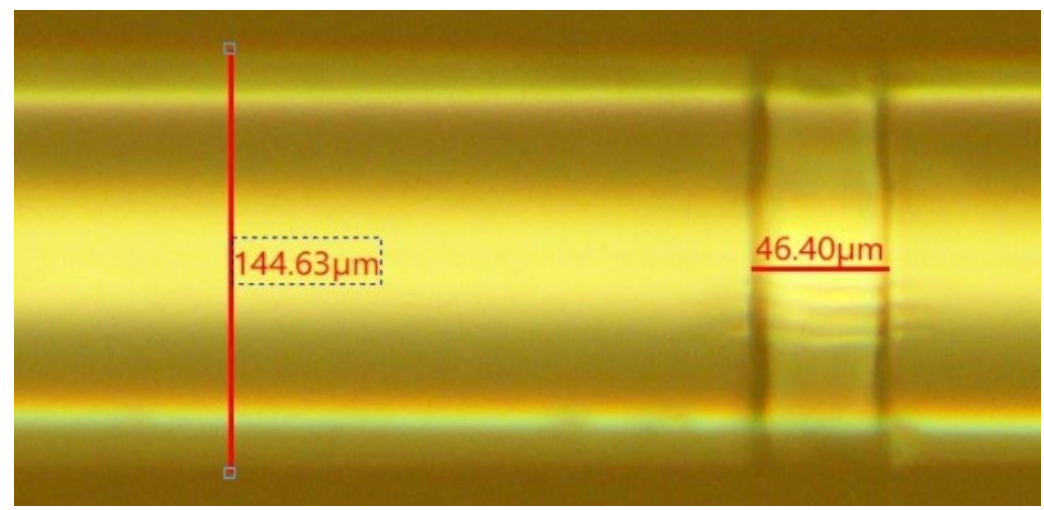

Figure A.3.5 Heated and Spray coated SU-8 was deposited and exposed through the Aligner. 

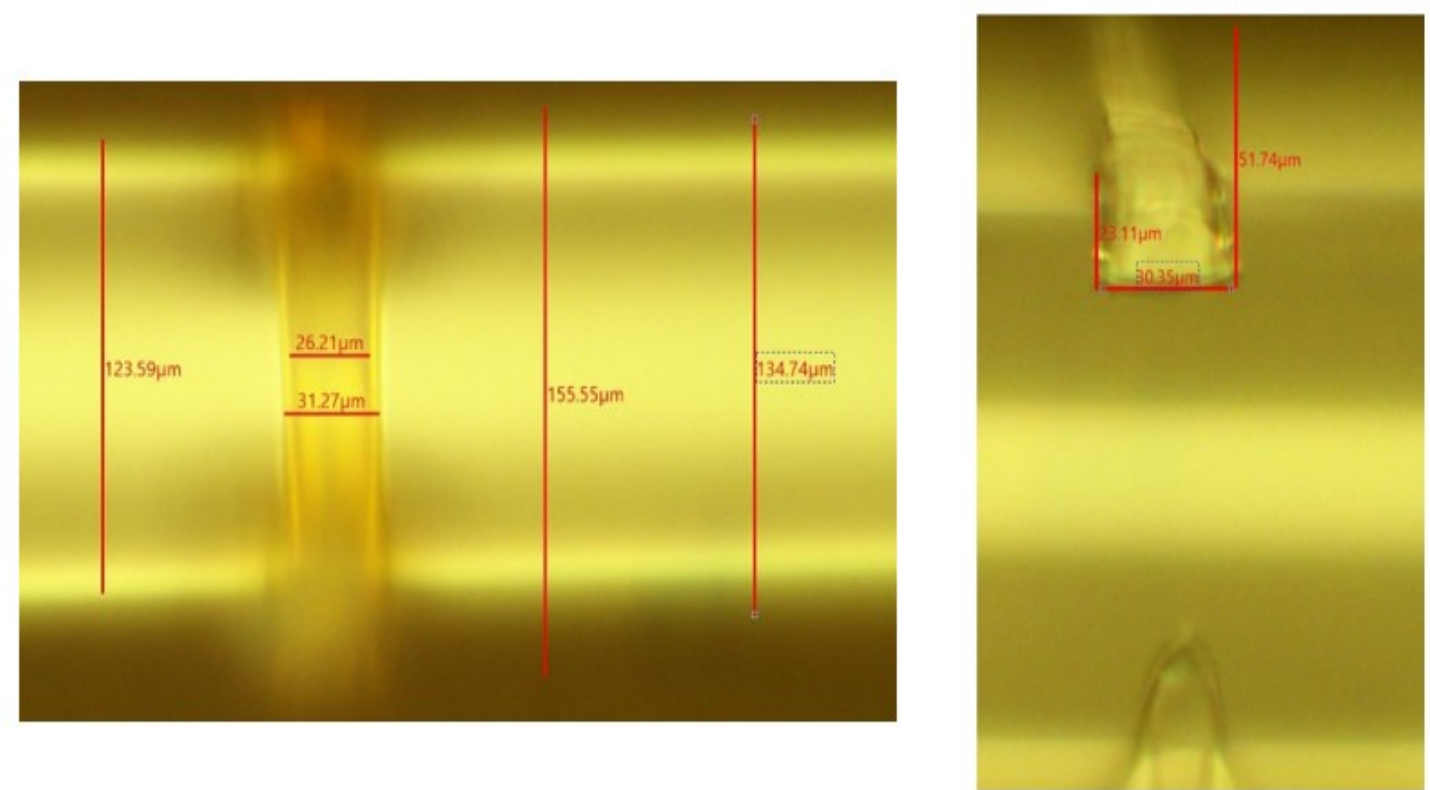

Figure A.3.6 Left side is the front view of the fiber just as it was placed in the aligner and the right side is the edge view, hard baked to finish the photolithographic process.

Another attempt was made into the development of the resonator where $\mathrm{O}_{2}$ plasma preening was used. The plasma preening system is operated by flowing a process gas, usually oxygen, through the chamber using microwave energy to clean organic and inorganic contaminations from the fiber. Then heated SU-8-2 was sprayed. Before and after, the deposition process will follow the same procedure as mentioned in Section A.2. The sample is exposed using SF-100; the final waveguides in Figure A.3.7 may not be a good option. All baking times used in this process are displayed in Table A.3.1. 


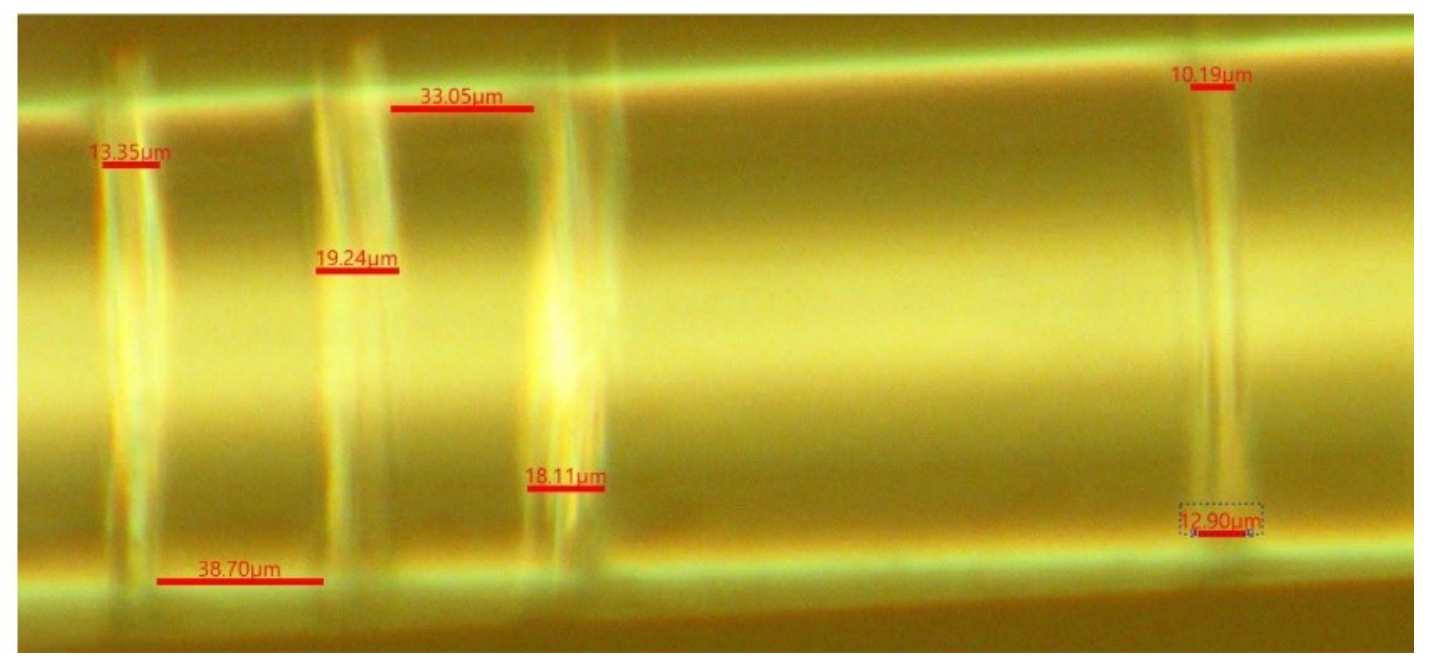

Figure A.3.7 $\mathrm{O}_{2}$ Plasma preen heated $S U-8-2$ is sprayed using SF-100 for exposure. 
Table A.3.1 Summary of the main steps followed during the photolithography process.

\begin{tabular}{|c|c|c|c|c|c|}
\hline $\begin{array}{c}\text { Deposition } \\
\text { method }\end{array}$ & $\begin{array}{c}\text { Pre-bake } \\
\text { time }\end{array}$ & $\begin{array}{c}\text { Exposure } \\
\text { time }\end{array}$ & PEB time & $\begin{array}{l}\text { Development } \\
\text { time (sec) }\end{array}$ & $\begin{array}{c}\text { Figure } \\
\#\end{array}$ \\
\hline $\begin{array}{c}\text { Hand } \\
\text { painted }\end{array}$ & $\begin{array}{l}1-\min 65 \\
{ }^{\circ} \mathrm{C} \& 3- \\
\min 95^{\circ} \mathrm{C}\end{array}$ & $\begin{array}{l}\text { SF-100 } \\
7 \mathrm{sec}\end{array}$ & $\begin{array}{l}1-\min 65 \\
{ }^{\circ} \mathrm{C} \& 1- \\
\min 95^{\circ} \mathrm{C}\end{array}$ & 60 & A.3.1 \\
\hline Dipped & $\begin{array}{l}1-\min 65 \\
{ }^{\circ} \mathrm{C} \& 3- \\
\min 95^{\circ} \mathrm{C}\end{array}$ & $\begin{array}{l}\text { SF-100 } \\
17 \mathrm{sec}\end{array}$ & $\begin{array}{l}1-\min 65 \\
{ }^{\circ} \mathrm{C} \& 1- \\
\min 95^{\circ} \mathrm{C}\end{array}$ & 60 & A.3.3 \\
\hline Sprayed & $\begin{array}{l}1-\min 65 \\
{ }^{\circ} \mathrm{C} \& 3- \\
\min 95^{\circ} \mathrm{C}\end{array}$ & $\begin{array}{l}\text { SF-100 } \\
17 \mathrm{sec}\end{array}$ & $\begin{array}{l}1-\min 65 \\
{ }^{\circ} \mathrm{C} \& 1- \\
\min 95^{\circ} \mathrm{C}\end{array}$ & 60 & A.3.4 \\
\hline $\begin{array}{l}\text { Heated \& } \\
\text { Sprayed }\end{array}$ & $\begin{array}{l}5-\min 65 \\
{ }^{\circ} \mathrm{C} \& 10- \\
\min 95^{\circ} \mathrm{C}\end{array}$ & $\begin{array}{l}\text { Aligner } \\
30 \mathrm{sec}\end{array}$ & $\begin{array}{l}5-\min 65 \\
{ }^{\circ} \mathrm{C} \& 15- \\
\min 95^{\circ} \mathrm{C}\end{array}$ & 90 & $\begin{array}{l}\text { A.3.5- } \\
\text { A.3.6 }\end{array}$ \\
\hline $\begin{array}{c}\text { Heated \& } \\
\text { Sprayed }\left(\mathrm{O}_{2}\right. \\
\text { preen })\end{array}$ & $\begin{array}{l}1-\min 65 \\
{ }^{\circ} \mathrm{C} \& 3- \\
\min 95^{\circ} \mathrm{C}\end{array}$ & $\begin{array}{l}\text { SF-100 } \\
16 \mathrm{sec}\end{array}$ & $\begin{array}{l}1-\min 65 \\
{ }^{\circ} \mathrm{C} \& 1- \\
\min 95^{\circ} \mathrm{C}\end{array}$ & 80 & A.3.7 \\
\hline
\end{tabular}




\section{Appendix B}

The matrix populating expressions make use of several precomputed integral tables. The radial extent normalized integrals are provided here.

$$
\begin{gathered}
S_{a}\left(p_{f}, p^{*}\right)=2 \int_{0}^{1} J_{1}\left(\rho_{p_{f}} r\right) J_{o}\left(\rho_{p^{*}} r\right) r^{a} d r \\
T_{a}\left(p_{f}, p_{\Omega}, p^{*}\right)=2 \int_{0}^{1} J_{o}\left(\rho_{p_{f}} r\right) J_{o}\left(\rho_{p_{\Omega}} r\right) J_{o}\left(\rho_{p^{*}} r\right) r^{a} d r \\
U_{a}\left(p_{f}, p_{\Omega}, p^{*}\right)=2 \int_{0}^{1} J_{1}\left(\rho_{p_{f}} r\right) J_{o}\left(\rho_{p_{\Omega}} r\right) J_{o}\left(\rho_{p^{*}} r\right) r^{a} d r \\
V_{a}\left(p_{f}, p^{*}\right)=2 \int_{0}^{1} J_{o}\left(\rho_{p_{f}} r\right) J_{o}\left(\rho_{p^{*}} r\right) r^{a} d r
\end{gathered}
$$




\section{Bibliography}

[1] Vahala, K. Optical microcavities. Nature 424, 839-846 (2003)

[2] Birks, T.A, J.C Knight, and T.E Dimmick. "High-Resolution Measurement of the Fiber Diameter Variations Using Whispering Gallery Modes and No Optical Alignment." IEEE photonics technology letters 12, no. 2 (2000): 182-183.

[3] Little, B.E, J.S Foresi, G Steinmeyer, E.R Thoen, S.T Chu, H.A Haus, E.P Ippen, L.C Kimerling, and W Greene. "Ultra-Compact Si-SiO2 Microring Resonator Optical Channel Dropping Filters.” IEEE photonics technology letters 10, no. 4 (1998): 549-551.

[4] Zhang Meng, Wenlei Yang, Ke Tian, Jibo Yu, Angzhen Li, Shunbin Wang, Elfed Lewis, Gerald Farrell, Libo Yuan, and Pengfei Wang. "In-Fiber Whispering-Gallery Mode Microsphere Resonator-Based Integrated Device.” Optics letters 43, no. 16 (2018): 3961-3964.

[5] Gu, Fuxing, Fuming Xie, Xing Lin, Shuangyi Linghu, Wei Fang, Heping Zeng, Limin Tong, and Songlin Zhuang. "Single Whispering-Gallery Mode Lasing in Polymer Bottle Microresonators via Spatial Pump Engineering." Light, science \& applications 6, no. 10 (2017): e17061-e17061.

[6] Merrer, Pierre-Henri, Khaldoun Saleh, Olivier Llopis, Simone Berneschi, Franco Cosi, and Gualtiero Nunzi Conti. "Characterization Technique of Optical Whispering Gallery Mode Resonators in the Microwave Frequency Domain for Optoelectronic Oscillators." Applied Optics 51, no. 20 (2012): 4742-4748.

[7] Xiao, Sanshui, Liu, and Min Qiu. "Resonator Channel Drop Filters in a Plasmon-Polaritons Metal.” Optics express 14, no. 7 (2006): 2932-2937.

[8] Yang, Guoqing, Zheng Tan, Bichen Zou, and Yifu Zhu. "Interference Control of Nonlinear Excitation in a Multi-Atom Cavity Quantum Electrodynamics System." Optics letters 39, no. 23 (2014): 6695-6698. 
[9] O’Shea, D, C Junge, M Poellinger, A Vogler, and A Rauschenbeutel. “All-Optical Switching and Strong Coupling Using Tunable Whispering-Gallery-Mode Microresonators.” Applied physics. B, Lasers and optics 105, no. 1 (2011): 129-148.

[10] Asano. Motoki, Yuki Takeuchi, Weijian Chen, Şahin Kaya Özdemir, Rikizo Ikuta, Nobuyuki Imoto, Lan Yang, and Takashi Yamamoto. "Observation of Optomechanical Coupling in a Microbottle Resonator." Laser \& photonics reviews 10, no. 4 (2016): 603-611.

[11] Yariv, Amnon., Pocji. Yeh, and Amnon. Yariv. Photonics: Optical Electronics in Modern Communications 6th ed. New York: Oxford University Press, 2007

[12] Notomi, M, E Kuramochi, and H Taniyama. "Ultrahigh-Q Nanocavity with 1D Photonic Gap.” Optics express 16, no. 15 (2008): 11095-11102.

[13] Rayleigh. "The Problem of the Whispering Gallery." Philosophical magazine (London, England: 1945) 20, no. 120 (1910): 1001-.

[14] Oraevsky, Anatolii N. "Whispering-Gallery Waves." Quantum electronics (Woodbury, N.Y.) 32 , no. 5 (2002): 377-400.

[15] Tobing, Landobasa Y.M, and Pieter Dumon. "Fundamental Principles of Operation and Notes on Fabrication of Photonic Microresonators." In Photonic Microresonator Research and Applications, 1-27. Boston, MA: Springer US, 2010.

[16] Filatov, Yuri V, Alexander S Kukaev, Egor V Shalymov, and Vladimir Yu Venediktov. "Study of Cross-Sensitivity of Whispering Gallery Modes in Bottle Resonators to Rotation.” Optical engineering 57, no. 10 (2018): 107107-107107.

[17] Gu, Jinyi, Zhuo Zhang, Mi Li, and Yuejiang Song. "Mode Characteristics of Metal-Coated Microcavity." Physical review. A, Atomic, molecular, and optical physics 90, no. 1 (2014). 
[18] Pongsanam, P, P Juleang, S Mitatha, and P. P Yupapin. "Novel Optical Cryptography Using LIP Signals Generated by a PANDA Ring Resonator." Microwave and optical technology letters 53, no. 11 (2011): 2575-2580.

[19] Zamora, Vanessa, Antonio Diez, Miguel V Andres, and Benito Gimeno. "Refractometric Sensor Based on Whispering-Gallery Modes of Thin Capillaries." Optics express 15, no. 19 (2007): 12011-12016.

[20] Farnesi, Daniele, Franco Cosi, Cosimo Trono, Giancarlo C Righini, Gualtiero Nunzi Conti, and Silvia Soria. "Stimulated Anti-Stokes Raman Scattering Resonantly Enhanced in Silica Microspheres.” Optics letters 39, no. 20 (2014): 5993-5996.

[21] Sumetsky, M. "Whispering-Gallery-Bottle Microcavities: The Three-Dimensional Etalon." Optics letters 29, no. 1 (2004): 8-10.

[22] Wang, Jie, Fang Bo, Shuai Wan, Wuxia Li, Feng Gao, Junjie Li, Guoquan Zhang, and Jingjun Xu. "High-Q Lithium Niobate Microdisk Resonators on a Chip for Efficient ElectroOptic Modulation.” Optics express 23, no. 18 (2015): 23072-23078.

[23] ahala, K. J, S. M Spillane, D. K Armani, and T. J Kippenberg. "Ultra-High- Q Toroid Microcavity on a Chip.” Nature (London) 421, no. 6926 (2003): 925-928.

[24] Huang, He, "Novel Sensing Mechanisms for Chemical and Bio-sensing Using Whispering Gallery Mode Micro resonators" (2018). Engineering and Applied Science Theses \& Dissertations. 327

[25] Righini, Giancarlo C., and Silvia Soria. Advanced Sensing with Micro-Optical WhisperingGallery-Mode Resonators Bellingham, Washington: SPIE Press, 2017. 
[26] Hanumegowda, Niranjan M, Caleb J Stica, Bijal C Patel, Ian White, and Xudong Fan. “Refractometric Sensors Based on Microsphere Resonators.” Applied physics letters 87, no. 20 (2005): 201107-201107-3.

[27] Jat, Yusra, and Robert C Gauthier. "Micro-Bottle Resonator as Sensors Using Whispering Gallery Modes.” 11266:112661H-112661H-7. SPIE, 2020.

[28] Mazzei, A, S Götzinger, De S Menezes, G Zumofen, O Benson, and V Sandoghdar. “Controlled Coupling of Counterpropagating Whispering-Gallery Modes by a Single Rayleigh Scatterer: A Classical Problem in a Quantum Optical Light.” Physical review letters 99, no. 17 (2007): 173603-173603.

[29] Kippenberg, T.J, S.M Spillane, and K.J Vahala. "Modal Coupling in Traveling-Wave Resonators." Optics letters 27, no. 19 (2002): 1669-1671.

[30] Kippenberg, Tobias J. "Particle Sizing by Mode Splitting." Nature photonics 4, no. 1 (2010): $9-10$.

[31] Ozdemir, Sahin Kaya, Lan Yang, Da-Ren Chen, Yun-Feng Xiao, Lin Li, Jiangang Zhu, and Lina He. "On-Chip Single Nanoparticle Detection and Sizing by Mode Splitting in an UltrahighQ Microresonator." Nature photonics 4, no. 1 (2010): 46-49.

[32] Weiss, D.S, V Sandoghdar, J Hare, V Lef È Vre-Seguin, J.-M Raimond, and S Haroche. "Splitting of High-q Mie Modes Induced by Light Backscattering in Silica Microspheres." Optics letters 20, no. 18 (1995): 1835-1837.

[33] Chantada, L, N.I Nikolaev, A.L Ivanov, P Borri, and W Langbein. "Optical Resonances in Microcylinders: Response to Perturbations for Biosensing." Journal of the Optical Society of America. B, Optical physics 25, no. 8 (2008): 1312-1321. 
[34] He, L, Y.-F Xiao, C Dong, J Zhu, V Gaddam, and L Yang. “Compensation of Thermal Refraction Effect in High- Q Toroidal Microresonator by Polydimethylsiloxane Coating." Applied physics letters 93, no. 20 (2008): 201102-201102-3.

[35] Foreman, Matthew R, Wei-Liang Jin, and Frank Vollmer. “Optimizing Detection Limits in Whispering Gallery Mode Biosensing.” Optics express 22, no. 5 (2014): 5491-5511.

[36] Jiang, Xue-Feng, Yun-Feng Xiao, Chang-Ling Zou, Lina He, Chun-Hua Dong, Bei-Bei Li, Yan Li, Fang-Wen Sun, Lan Yang, and Qihuang Gong. "Highly Unidirectional Emission and Ultralow-Threshold Lasing from On-Chip Ultrahigh-Q Microcavities.” Advanced materials (Weinheim) 24, no. 35 (2012): OP260-OP264.

[37] Shao, Linbo, Xue-Feng Jiang, Xiao-Chong Yu, Bei-Bei Li, William R Clements, Frank Vollmer, Wei Wang, Yun-Feng Xiao, and Qihuang Gong. "Detection of Single Nanoparticles and Lentiviruses Using Microcavity Resonance Broadening." Advanced materials (Weinheim) 26, no. 7 (2014): 991-991.

[38] Kipp, T, H Welsch, Ch Strelow, Ch Heyn, and D Heitmann. “Optical Modes in Semiconductor Microtube Ring Resonators.” Physical review letters 96, no. 7 (2006): 077403077403.

[39] Zervas, M.N, J.S Wilkinson, and G.S Murugan. "Experimental Demonstration of a Bottle Microresonator." In 2009 Conference on Lasers and Electro-Optics and 2009 Conference on Quantum Electronics and Laser Science Conference, 1-2. IEEE, 2009.

[40] Senthil Murugan, G, M.N Petrovich, Y Jung, J.S Wilkinson, and M.N Zervas. "HollowBottle Optical Microresonators.” Optics express 19, no. 21 (2011): 20773-20784. 
[41] Zervas, M.N, G.S Murugan, and J.S Wilkinson. "Demonstration of Novel High-Q Fibre WGM 'Bottle' Microresonators.” In 2008 10th Anniversary International Conference on Transparent Optical Networks, 4:58-60. IEEE, 2008.

[42] Kakarantzas, G, T.E Dimmick, T.A Birks, R Le Roux, and P.St.J Russell. “Miniature AllFiber Devices Based on $\mathrm{CO}_{2}$ Laser Microstructuring of Tapered Fibers.” Optics letters 26, no. 15 (2001): 1137-1139.

[43] Murugan, Ganapathy Senthil, James S Wilkinson, and Michalis N Zervas. "Selective Excitation of Whispering Gallery Modes in a Novel Bottle Microresonator." Optics express 17, no. 14 (2009): 11916-11925

[44] Wang, Jiao, Tianrong Zhan, Gaoshan Huang, Paul K Chu, and Yongfeng Mei. "Optical Microcavities with Tubular Geometry: Properties and Applications: Tubular Optical Microcavity." Laser \& photonics reviews 8, no. 4 (2014): 521-547.

[45] Lu, Qijing, Xiaogang Chen, Shusen Xie, and Xiang Wu. "Controllable and Selective SingleMode Lasing in Polymer Microbottle Resonator.” Optics express 26, no. 16 (2018): 2018320191.

[46] Santamaría-Botello, G.A, L.E García Muñoz, F Sedlmeir, S Preu, D Segovia-Vargas, K Atia Abdalmalak, S Llorente Romano, et al. "Maximization of the Optical Intra-Cavity Power of Whispering-Gallery Mode Resonators via Coupling Prism.” Optics express 24, no. 23 (2016): $26503-26514$.

[47] Ilchenko, Vladimir S, X. Steve Yao, and Lute Maleki. "Pigtailing the High-Q Microsphere Cavity: A Simple Fiber Coupler for Optical Whispering-Gallery Modes.” Optics letters 24, no. 11 (1999): 723-725. 
[48] Gu, Fuxing, Fuming Xie, Xing Lin, Shuangyi Linghu, Wei Fang, Heping Zeng, Limin Tong, and Songlin Zhuang. "Single Whispering-Gallery Mode Lasing in Polymer Bottle Microresonators via Spatial Pump Engineering." Light, science \& applications 6, no. 10 (2017): e17061-e17061.

[49] Vlasov, Yurii, Fengnian Xia, and Lidija Sekaric. "Ultracompact Optical Buffers on a Silicon Chip." Nature photonics 1, no. 1 (2007): 65-71.

[50] Sumetsky, M. "Delay of Light in an Optical Bottle Resonator with Nanoscale Radius Variation: Dispersionless, Broadband, and Low Loss.” Physical review letters 111, no. 16 (2013): $163901-163901$.

[51] Scheucher, Michael, Adèle Hilico, Elisa Will, Jürgen Volz, and Arno Rauschenbeutel. "Quantum Optical Circulator Controlled by a Single Chirally Coupled Atom.” Science (American Association for the Advancement of Science) 354, no. 6319 (2016): 1577-1580.

[52] O’Shea, D, C Junge, M Poellinger, A Vogler, and A Rauschenbeutel. “All-Optical Switching and Strong Coupling Using Tunable Whispering-Gallery-Mode Microresonators.” Applied physics. B, Lasers and optics 105, no. 1 (2011): 129-148.

[53] Bianucci, Pablo. “Optical Microbottle Resonators for Sensing.” Sensors (Basel, Switzerland) 16, no. 11 (2016): 1841-.

[54] Stoian, Razvan-Ionut, Khoa V Bui, and A T Rosenberger. "Silica Hollow Bottle Resonators for Use as Whispering Gallery Mode Based Chemical Sensors.” Journal of optics (2010) 17, no. 12 (2015): 125011-.

[55] Johari, M.A.M, A Al Noman, M.I.M Abdul Khudus, M.H Jali, H.H.M Yusof, S.W Harun, and M Yasin. "Microbottle Resonator for Formaldehyde Liquid Sensing." Optik (Stuttgart) 173 (2018): 180-184. 
[56] Hou, Fengyu, Xiaobei Zhang, Zijie Wang, Lei Yang, Wen Sun, Yong Yang, Yanhua Dong, Yi Huang, and Tingyun Wang. "Magnetic Fluid Infiltrated Microbottle Resonator Sensor With Axial Confined Mode.” IEEE photonics journal 12, no. 5 (2020): 1-9.

[57] Suh, Jeewon, Kewen Han, Christopher W Peterson, and Gaurav Bahl. "Invited Article: RealTime Sensing of Flowing Nanoparticles with Electro-Opto-Mechanics." APL photonics 2, no. 1 (2017): 10801-010801-7.

[58] Chenari, Zeinab, Hamid Latifi, and Mostafa Peysokhan. "Nanoparticle Detection by Mode Splitting in Hollow Bottle Microresonators.” Journal of nanophotonics 10, no. 1 (2016): 016009016009.

[59] Yang, Yong, Sunny Saurabh, Jonathan M Ward, and Śile Nic Chormaic. "High-Q, UltrathinWalled Microbubble Resonator for Aerostatic Pressure Sensing." Optics express 24, no. 1 (2016): 294-299.

[60] Nemova, Galina, and Raman Kashyap. "Silica Bottle Resonator Sensor for Refractive Index and Temperature Measurements.” Sensors (Basel, Switzerland) 16, no. 1 (2016): 87-.

[61] Persichetti, G, I.A Grimaldi, G Testa, and R Bernini. "Self-Assembling and Packaging of Microbottle Resonators for All-Polymer Lab-on-Chip Platform.” Sensors and actuators. A. Physical. 280 (2018): 271-276.

[62] Sumetsky, M. "Slow Light Optofluidics: A Proposal." Optics letters 39, no. 19 (2014): $5578-5581$.

[63] Nemova, Galina, and Raman Kashyap. "Bottle Micro-Resonator Temperature Sensors for Laser Coolers." In Proceedings of SPIE - The International Society for Optical Engineering, 9765:97650M-97650M-7. SPIE, 2016. 
[64] Lavín, Álvaro, Jesús de Vicente, Miguel Holgado, María F Laguna, Rafael Casquel, Beatriz Santamaría, María Victoria Maigler, Ana L Hernández, and Yolanda Ramírez. "On the Determination of Uncertainty and Limit of Detection in Label-Free Biosensors.” Sensors (Basel, Switzerland) 18, no. 7 (2018): 2038-.

[65] Zhang, Xingwang, Liying Liu, Lei Xu, Xudong Fan, and Guangya Zhou. “Optical Microand Nanoresonators for Biochemical Sensing." In Optical MEMS, Nanophotonics, and Their Applications, 301-320. 1st ed. CRC Press, 2018.

[66] Cai, Zhiping, Alain Chardon, Huiying Xu, Patrice Féron, and Guy Michel Stéphan. "Laser Characteristics at $1535 \mathrm{Nm}$ and Thermal Effects of an Er:Yb Phosphate Glass Microchip Pumped by Ti:sapphire Laser." Optics communications 203, no. 3 (2002): 301-313.

[67] Herter, Jonas, Valentin Wunderlich, Christian Janeczka, and Vanessa Zamora. "Experimental Demonstration of Temperature Sensing with Packaged Glass Bottle Microresonators.” Sensors (Basel, Switzerland) 18, no. 12 (2018): 4321-.

[68] Batumalay, Malathy, Md Ashadi Md Johari, Muhammad Imran Mustafa Abdul Khudus, Mohd Hafiz Bin Jali, Abdullah Al Noman, and Sulaiman Wadi Harun. "Microbottle Resonator for Temperature Sensing." Journal of physics. Conference series 1371, no. 1 (2019): 12006-.

[69] Bhattacharya, Shubhayan, Aneesh V Veluthandath, Ganapathy Senthil Murugan, and Prem B Bisht. "Temperature Dependence of Whispering Gallery Modes of Quantum Dot-Doped Microbottle Resonators.” Journal of luminescence 221 (2020): 117050-.

[70] Hu, Jinliang, Sheng Liu, Xiang Wu, Liying Liu, and Lei Xu. “Orthogonal Demodulation Pound-Drever-Hall Technique for Ultra-Low Detection Limit Pressure Sensing." Sensors (Basel, Switzerland) 19, no. 14 (2019): 3223-. 
[71] Yang, Yong, Jonathan Ward, and Síle Nic Chormaic. "Quasi-Droplet Microbubbles for High Resolution Sensing Applications.” Optics express 22, no. 6 (1722): 6881-6898.

[71] Berneschi, S, D Farnesi, F Cosi, G. Nunzi Conti, S Pelli, G.C Righini, and S Soria. "High Q Silica Microbubble Resonators Fabricated by Arc Discharge.” Optics letters 36, no. 17 (2011): $3521-3523$.

[73] Tang, Ting, Xiang Wu, Liying Liu, and Lei Xu. "Packaged Optofluidic Microbubble Resonators for Optical Sensing.” Applied optics (2004) 55, no. 2 (2016): 395-399.

[74] Johari, Md Ashadi Md, Muhammad Imran Mustafa Abdul Khudus, Mohd Hafiz Bin Jali, M.S Maslinda, Ummu Umairah Mohamad Ali, Sulaiman Wadi Harun, A.H Zaidan, R Apsari, and M Yasin. "Effect of Tapering Diameters with Microbottle Resonator for Formaldehyde (CH2O) Liquid Sensing.” Sensing and Bio-Sensing Research 25 (2019): 100292-.

[75] Stoian, Razvan-Ionut, Barry K Lavine, and A.T Rosenberger. "pH Sensing Using Whispering Gallery Modes of a Silica Hollow Bottle Resonator.” Talanta (Oxford) 194 (2019): $585-590$.

[76] Cardoso, José Roberto. Electromagnetics through the Finite Element Method: A Simplified Approach Using Maxwell's Equations. CRC Press, 2017.

[77] Gedney, Stephen Douglas. Introduction to the Finite-Difference Time-Domain (FDTD) Method for Electromagnetics. San Rafael, Calif. (1537 Fourth Street, San Rafael, CA 94901 USA): Morgan \& Claypool, 2011.

[78] Newman, Scott Ronald. "Fourier-Bessel Analysis of Photonic Crystals and Photonic Quasicrystals" Thesis (Ph.D.) - Carleton University, 2013., 2013. 
[79] Newman, Scott R, and Robert C Gauthier. "Fourier-Bessel Analysis of Localized States and Photonic Bandgaps in 12-Fold Photonic Quasi-Crystals.” Journal of the Optical Society of America. A, Optics, image science, and vision 29, no. 11 (2012): 2344-2349.

[80] Kane Yee. “Numerical Solution of Initial Boundary Value Problems Involving Maxwell's Equations in Isotropic Media." IEEE transactions on antennas and propagation 14, no. 3 (1966): $302-307$.

[81] Taflove A, Umashankar KR. Solution of Complex Electromagnetic Penetration and Scattering Problems in Unbounded Regions. In Computational Methods for Infinite Domain Media-Structure Interactions. American Society of Mechanical Engineers.Vol. 46. (1981) p. 83113

[82] Umashankar, Korada, and Allen Taflove. "A Novel Method to Analyze Electromagnetic Scattering of Complex Objects." IEEE transactions on electromagnetic compatibility EMC-24, no. 4 (1982): 397-405.

[83] Taflove. Allen, and Susan C. Hagness. Computational Electrodynamics: The FiniteDifference Time-Domain Method. 3rd ed Boston: Artech House, 2005.

[84] Bérenger, Jean-Pierre. Perfectly Matched Layer (PML) for Computational Electromagnetics. Synthesis Lectures on Computational Electromagnetics. 1st ed. Vol. 8. San Rafael, Calif. (1537 Fourth Street, San Rafael, CA 94901 USA): Morgan \& Claypool Publishers, 2007.

[85] Saleh, Bahaa E. A., and Malvin Carl Teich. Fundamentals of Photonics Third edition. Hoboken, New Jersey: Wiley, 2019. 
[86] Lourtioz, Jean-Michel., Henri. Benisty, Vincent. Berger, Jean-Michel. Gerard, Daniel. Maystre, and Alexei. Tchelnokov. Photonic Crystals Towards Nanoscale Photonic Devices 2nd ed. 2008. Berlin, Heidelberg: Springer Berlin Heidelberg, 2008.

[87] Guan, Shuguang, C.-H Lai, and G.W Wei. "Fourier-Bessel Analysis of Patterns in a Circular Domain.” Physica. D 151, no. 2 (2001): 83-98.

[88] Zana, Y, R.M Cesar-Jr, R Feris, and M Turk. "Local Approach for Face Verification in Polar Frequency Domain.” Image and vision computing 24, no. 8 (2006): 904-913.

[89] McGloin, D, and K Dholakia. "Bessel Beams: Diffraction in a New Light.” Contemporary physics 46, no. 1 (2005): 15-28.

[90] Felbacq, D, G Tayeb, and D Maystre. "Scattering by a Random Set of Parallel Cylinders." Journal of the Optical Society of America. A, Optics, image science, and vision 11, no. 9 (1994): 2526-.

[91] Gauthier, Robert C. "Reformulation of the Fourier-Bessel Steady State Mode Solver." Optics communications 375 (2016): 63-71.

[92] Gauthier, Robert C. "Defining Cylindrical Space Optical Resonators through Supported Mode Properties: Inverse Numerical Process.” Optics express 26, no. 8 (2018): 10091-10108. [93] Gauthier, Robert C. "Structural Disorder Correlation Examined Using the Fourier-Bessel Technique." Optics communications 355 (2015): 504-515.

[94] Newman, Scott R, and Robert C Gauthier. "Fourier-Bessel Analysis of Localized States and Photonic Bandgaps in 12-Fold Photonic Quasi-Crystals.” Journal of the Optical Society of America. A, Optics, image science, and vision 29, no. 11 (2012): 2344-2349. 
[95] Gauthier, Robert C, Mohammed A Alzahrani, and Seyed Hamed Jafari. “Theoretical Examination of the Slot Channel Waveguide Configured in a Cylindrically Symmetric Dielectric Ring Profile.” Optics communications 329 (2014): 154-162.

[96] Gauthier, Robert C. "Reformulation of the Fourier-Bessel Steady State Mode Solver." Optics communications 375 (2016): 63-71.

[97] Gauthier, Robert C. “Anisotropic Resonator Analysis Using the Fourier-Bessel Mode Solver." Optics communications 410 (2018): 317-327.

[98] Besserer, George J, and Donald B Robinson. "Refractive Indexes of Ethane, Carbon Dioxide, and Isobutane.” Journal of chemical and engineering data 18, no. 2 (1973): 137-140.

[99] Temperature Coefficient of the Refractive Index SCHOTT Technical Information TIE-19

[100] Shukla, R K, Preeti Misra, Sweta Sharma, Nagendra Tomar, and Pratima Jain. "Refractive Index and Molar Refractivity of Benzonitrile, Chlorobenzene, Benzyl Chloride and Benzyl Alcohol with Benzene at $\mathrm{T}=298.15,303.15,308.15$ and 313.15 K." Journal of the Iranian Chemical Society 9, no. 6 (2012): 1033-1043. 